\title{
RESEARCH IN PARTICLE PHYSICS
}

Grant No. DE-FG02-91ER40676

\author{
Boston University \\ Boston, Massachusetts 02215 \\ August, 1993
}

DISCLAIMER

This report was prepared as an account of work sponsored by an agency of the United States Government. Neither the United States Government nor any agency thereof, nor any of their employees, makes any warranty, express or implied, or assumes any legal liability or responsibility for the accuracy, completeness, or usefulness of any information, apparatus, product, or process disciosed, or represents that its use would not infringe privately owned rights. Reference herein to any specific commercial product, process, or service by trade name, trademark, manufacturer, or otherwise does not necessarily constitute or imply its endorsement, recommendation, or favoring by the United States Government or any agency thereof. The views and opinions of authors expressed herein do not necessarily state or reflect those of the United States Government or any agency thereof.

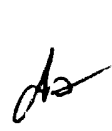

OHSTRIBUTIOH OF THIS DOCUMENT IS UNLIMITEB

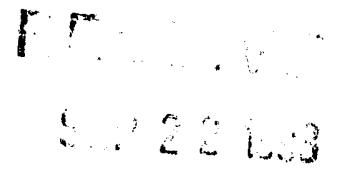

$(-4)$ 


\section{TABLE OF CONTENTS}

Introduction and Description of Facilities $\ldots \ldots \ldots \ldots \ldots \ldots \ldots \ldots \ldots \ldots$

Task A: Colliding Beams Project $\ldots \ldots \ldots \ldots \ldots \ldots \ldots \ldots \ldots \ldots \ldots \ldots \ldots \ldots \ldots$

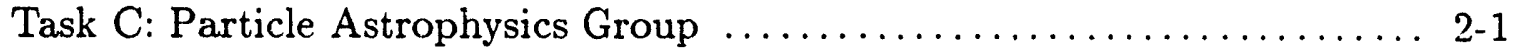

Task D: Neutrino Astroparticle Physics and Proton Decay............. 3-1

Task E: Theoretical Particle Physics $\ldots \ldots \ldots \ldots \ldots \ldots \ldots \ldots \ldots \ldots \ldots \ldots \ldots \ldots$

Task F: Muon g-2 Experiment at BNL $\ldots \ldots \ldots \ldots \ldots \ldots \ldots \ldots \ldots \ldots \ldots \ldots \ldots \ldots$

Task J: Topics in Particle Physics......................... 6-1

Task K: Hadron Calorimetry for the GEM Detector .............. $7-1$

Task N: Topics in Elementary Particle Physics ................. 8-1

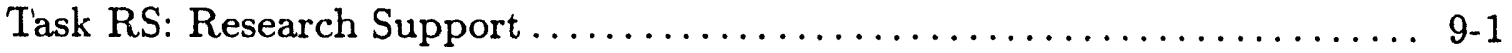




\section{INTRODUCTION}

This proposal presents the research accomplishments and ongoing activities of Boston University researchers in high energy physics. Some changes have been made in the structure of the program from the previous arrangement of tasks. Task B, Accelerator Design Physics, is being submitted as a separate proposal for an independent grant; this will be consistent with the nature of the research and the source of funding. We are active in seven principal areas:

- Task A: Colliding Beams - physics of $\mathrm{e}^{+} \mathrm{e}^{-}$and $\bar{p} \mathrm{p}$ collisions

- Task C: MACRO Experiment - search for magnetic monopoles and study of cosmic rays

- Task D: Proton Decay - search for nucleon instability and study of neutrino interactions

- Tasks E, J, and N: Particle Theory - theoretical high energy particle physics, including two Outstanding Junior Investigator awards

- Task F: Muon G-2 - measurement of the anomalous magnetic moment of the muon

- Task K: SSCintcal - calorimetry for the GEM Experiment

- Task L: Muon detectors for the GEM Experiment

There is a great deal of interaction between the participants in these projects and considerable overlap among the groups. The shared resources, facilities, and expertise available in this setting enhance the productivity of each program. Each task has designated Principal Investigators; overall management of the program is supplied by a Project Director and a Grant Administrator. Administration of the program is discussed under Task RS. This approach to research and management improves administrative efficiency and provides coherent oversight of all aspects of the program.

The body of the proposal is devoted to detailed discussions of each of the tasks. The total budget request for the program appears in a summary chapter that includes a general budget discussion and individual budget requests and explanations for each of the tasks. 


\section{DESCRIPTION OF FACILITIES}

The HEP research program at Boston University has received excellent support from the University in every aspect. Our research facilities, described in detail below, are upto-date and are superior to those to be found in most universities. The Physics Research Building that houses our research program was built to our specifications; it includes mechanical anci electronics shops and eight thousand-square-foot lab spaces. We have constructed a clean room for construction of particle detectors in a dust-free environment. The University has provided wel! over one million do!lars of capital funds to furnish the labs with the tools and instruments necessary for HEP research.

The research projects benefit enormously from the availability of excellent facilities support for mechanical and electronic design and fabrication and for computing. The following sections highlight the major aspects of these facilities.

\section{Mechanical Facilities}

The Scientific Instrument Facility is located in the basement of the Physics Research Building and is administered by Physics Department personnel. This facility is a superblyequipped machine shop with a staff of eight machinists and an assembly team of three technicians. The shop has 5,000 square feet of climate controlled workspac " with truck access, crane coverage, and a high bay area. The shop has a full complement of machines: vertical and horizontal mills, high-capacity lathes, high-precision lathes, a bcring mill, and a grinder. The shop also has all the machinery needed for stock preparation, including a large shear, an automatic cutoff saw, and a large bending brake. In addition, the shop has complete welding and leak-checking capabilities. A shop is only as good as the people who work there, and we are fortunate to have an outstanding team of skilled, experienced instrument makers. The shop director, George Pisiello, has over 30 years experience in scientific instrument design and fabrication; he is known throughout the nation for his expertise, particularly in high vacuum systems.

The other outstanding feature of the shop is the Computer Numerically Controlled equipment:

- Okuma MV3 machining center

- Okuma MV5 machining center

- Okuma Lathe 
- Tsugami Swiss Lathe.

To this array of equipment we have recently added a very large capacity, five-axis CNC Giddings and Lewis 4 " horizontal boring mill. This mill has a machining precision of better than 0.001 " over a travel of 84 " by 48 " by $60 "$.

These high precision, high capacity machines give ar facility a production capability both for quality and quantity - that is perhaps unequaled at any other university. All these CNC machines are connected to our CAD/CAE system, Anvil 5000 software running on a Sun 4,470 platform. The SIF staff are equipped with workstations at which the CNC programs are prepared and then downloaded to the machines on the shop floor. A custom post-processor facilitates the generation of machine codes. Of the staff of eight, two are fully occupied with the CNC side of the facility, and two more of the machinists have the ability to fill in when needed.

With this facility, we have been able to make major contributions to the fabrication of HEP experiments. We fabricated many components for the SLD experiment, including pieces for the drift chamber, the Cherenkov ring-imaging detectors, and the liquid argon calorimeter. Presently, a large fraction of the facility's capacity is devoted to work for the new muon g-2 ring being built at Brookhaven National Laboratory.

\section{Electronics Facilities}

The Electronics Design Facility is located on the fourth floor of the Physics Research Building. It has a staff of three engineers and three technicians, and regularly employs students as additional workers are required. The EDF offers advanced electronics design, prototyping, and testing capability in support of our research program. A major design tool is the electronics CAD package from Mentor, running on Apollo workstations. With this resource, circuits are not only designed but can also be simulated before any actual prototyping is done. Advanced analog and digital circuits have been designed and produced. Some examples include: for CP/LEAR a 64-channel TDC system with 100 psec resolution and digitization and readout of all channels in one microsecond; a $200 \mathrm{Megasample} / \mathrm{sec}$ waveform digitizer system for the MACRO experiment; and, currently in development, a GaAs ASIC digital TDC with continuous readout for the DUMAND experiment. The EDF has high-speed test equipment and operates a data acquisition system to be able to test experimental equipment. 


\section{Computing Facilities}

The computing environment in which we work is diverse and comprehensive, with many machines and many software resources. The Physics Department has two full-tine computing staff who maintain the systems and the software. Our computing facilities include the following machines:

- Vax 4000-300 plus various microvaxes and vaxstations

- Eight DECstations plus four Alpha AXP Model 3000 machines

- Sun 670MP server, Sparcstation 10 Model 41, and approximately 30 smaller Sun machines

- Connection Machine CM5, with 64 processors

- Silicon Graphics S440 Challenge

- Four Silicon Graphics workstations

- SGI 4D/340S and five SGI Indigo work stations.

The last item in this list designates the server and workstations dedicated to the HEP program and purchased with DOE funds in 1991. These machines need to be upgraded; a proposal will be prepared for submission in the Fall.

Our computational facilities are linked together by broad-band networks so that every office has a connection to every machine; we are linked by all the major networks to the rest of the world as well. 
TASK A: COLLIDING BEAMS PROJECT

\author{
Faculty: $\quad$ Professor Scott Whitaker \\ Research Assistant Professor James T. Shank \\ Research Associate: (replacement for A. S. Johnson) \\ Graduate Student: John Coller
}

\title{
Introduction
}

The Colliding Beams Group currently has research activities in two major areas: in the SLD project ai SLAC and in the GEM Experiment at the SSC. The SLD is running well, and the SLC is delivering beam with high polarization, high luminosity, and high reliability. The collaboration is proposing to continue for several years to amass a sample of approximately one million polarized $Z^{\circ}$ 's. With this data sample the SLD will provide world's-best measurements of electroweak parameters. In the SSC program, we have a major role within the GEM muon system, where we have been developing the Cathode Strip Chambers that are the technology of choice for muon measurements. The SSC program is in upheaval; we expect that it will go forward but on a stretched-out timescale leading to data on electroweak symmetry breaking being collected perhaps a decade from now. To complement this program we are developing a role in the Beijing Spectrometer (BES) experiment at the Beijing Electron Positron Collider. This experiment is providing copious data on the tau lepton and charmed particles. The very high statistics and excellent data quality that would be available after the proposed upgrades of the accelerator and the detector would open the door to a great deal of interesting physics in the tau/charm sector. We have a large role in the proposed upgrade of the Main Drift Chamber, where the exceptional machining facilities at Boston University give us a unique capability and will position us well to exploit this physics potential.

The staffing of the CB Group is changing; Research Assistant Professor Tony Johnson is departing to take a staff position at SLAC, effective August 1, 1993. We have a commitment to the SLD to replace Tony with a postdoc to be located at SLAC to work on the CRID system, so a replacement for Tony is requested and included in the proposed budget. 


\section{SLD Activities}

Within the SLD, our primary activity has been on the endcap CRID. As discussed below, we have constructed, installed, and commissioned the proportional wire detectors for the endcap ring-imaging Cherenkov counters. We are also a major contributor to the on-line and off-line software for the experiment. During the next several years we will collect and analyze data on the production and decays of $Z^{\circ}$ 's.

The SLD has been running for several years. The detector is operating well; the hardware and software systems are shaken down and sufficient data is in hand to tune the analysis codes. The SLC has made dramatic progress in quality and quantity of beam. Noise and backgrounds are down, and luminosity performance has continued to improve; we are now logging up to $40 Z^{\circ}$ 's per hour at peak, with typically $500 Z^{\circ}$ 's per day. The polarization of the electron beam is an important part of the SLC capability. It will make the SLD competitive with the LEP experiments in some physics topics despite the luminosity advantage of the CERN machine. The polarization of the electron beam at the interaction point has also improved dramatically: with the new electron source, typical electron beam polarization at the interaction point has been $63 \%$, compared to $22 \%$ during the last run. More than 40,000 "polarized" $Z^{\circ}$ 's have been logged during this run, and the experiment is on course to $\log$ well over the $50,000 Z^{\circ}$ goal before the end of this run.

Analysis of SLD data is proceeding well. Results for the left-right asymmetry $A_{L R}$ from last year's data have been published, and papers on QCD studies are ready for submission. For many of the detailed studies of the standard model, systematic errors dominate the experimental measurement. In these cases, the SLD will again be competitive with the LEP experiments.

The forward region of the SLD will be particularly important in the measurement of asymmetries. These measurements are a sensitive probe of the Standard Model and may give evidence for new particles beyond the kinematic range of direct production experiments. Coverage of the region between 10 and 35 degrees in polar angle significantly increases our asymmetry measurement capabilities. The endcap CRID (Cerenkov Ring Imaging Device) project is a central part of this program, and its timely completion will increase the capability of the SLD to exploit the competitive advantage that polarization gives us. 


\section{CRID Endcap Detectors}

The endcap CRID is a ring-imaging gas Cherenkov detector that will provide particle identification over a wide momentum range. Particles traversing the endcaps emit Cherenkov radiation in the freon radiator; the Cherenkov photons are focused by mirrors onto a quartz-walled drift box filled with a TMAE-laden gas, in which the photons interact to create single photoelectrons. The electrons drift to a picket fence proportional detector with 7 micron diameter carbon "wires" on a .138 inch pitch. Drift time measurement, wire location, and charge division along the wires permit reconstruction of the photon interaction point with millimeter precision in each coordinate. Reconstruction of the photoelectron positions allows us to determine the angle of emission of the Cherenkov radiation with respect to the track direction, yielding a velocity measurement. Combined with the momentum measurement from the drift chambers, this gives particle identification capability. The threshold for pions is $2.5 \mathrm{GeV} / \mathrm{c}$ and for kaons is $9 \mathrm{GeV} / \mathrm{c}$.

Five of the detectors have been installed in each endcap (North and South) of the SLD. Installation of high voltage, cooling water, heating circuits, gas supply, and readout electronics has been completed. Commissioning of these detectors is nearly completed, the remaining problems being associated with debugging the readout electronics and fixing cabling and connection problems. The $\mathrm{CO}_{2}$ /ethane drift gas system is operational. There is a shortage of sufficiently pure ethane, but supplies are being located. An independent freon radiator gas system has been developed for the endcaps so they can be operated independently of the barrel CRID system. The ECRID's have been taking data routinely, with TMAE introduced into the drift gas for the North endcap. We have begun to use the UV fiber optic system to establish the calibrations for the detectors. Laser pulses can be used to create ions along specific optic paths in the drift volumes, and reconstruction of these "events" can be matched to the known geometry to verify detector parameters. Figure 1 shows the detectors in the SLD. Figure 2 plots reconstructed positions of laserinduced events; the paths of the laser are clearly visible.

Operation of proportional chambers in the TMAE-laced gas is a very sensitive business. Small standing currents in the nanoampere range caused by regenerative breakdown due to the residue of TMAE oxidation products on the conducting surfaces can cause cumulative damage. In the barrel CRID, approximately $10 \%$ of the 40 detectors are now out of service because of broken wires attributed to electrostatic problems brought on 
by this process. To avert problems, we need to monitor the currents and to drop the high voltage if currents exceed a very low threshold - we typically set a current trip at $j$ nanoamperes. Monitoring high voltage currents at this level is very difficult. This difficulty is compounded by a regulatory problem with the CAEN high voltage supplies that, interacting with the $\mathrm{HV}$ filtering on the detectors, causes a roughly $1 \mathrm{~Hz} 10$ nanoampere oscillatory current. To overcome the deficiencies of the HV supplies and allow HV current monitoring we developed a circuit that combines an amplifying and digitizing stage at $\mathrm{HV}$, optical coupling to a ground-potential DAC, and long period ( 5 second) analog averaging. This work is similar to some work we are pursuing for the GEM muon detector HV system (see below). The new nanoammeter circuits are installed and give improved stability. Time history plots of the cathode current during changes in beam conditions show clear correlations with the activity in the endcap drift chambers.

Particle identification in the endcaps will rely on momentum measurements from the endcap drift chamber (ECDC). Considerable improvement in the performance of the ECDC has recently been achieved by tuning data acquisition parameters. Prospects are now encouraging for successful operation of the combined endcap systems. We have an active group of physicists developing the analysis software and integrating the drift chamber and CRID data.

\section{Software}

Over the past five years we have been heavily involved with the development of the online and offline software for the SLD experiment. In particular, A. S. Johnson has been a key member of the software team, designing and developing several major areas of the SLD off-line reconstruction and analysis code and supervising the development of all SLD software utilities.

SLD has emphasized the use of interactive data analysis tools to aid in the rapid anaiysis of data once it is obtained and to help in the development and debugging of Monte-Carlo and reconstruction programs. This has entailed the development of a number of innovative analysis tools, including:

- Advancement of the MORTRAN macro preprocessor for FORTRAN.

- Development of a data management system, JAZELLE.

- Development of the Distributed Update Control System DUCS.

- Development of a graphical user interface, the Motif Interactive Data Analysis Shell 
MIDAS.

These powerful utilities form the core of the SLD analysis system. Although Johnson will now be a SLAC staff member, we expect to work closely with him to use these utilities in the analysis of the endcap data.

\section{Planned SLD Activities}

With present conditions, the current run will yield in excess of 50,000 $Z^{\circ}$ 's with an average electron polarization of $63 \%$. Determination of the weak mixing angle from the leftright asymmetry measured with this sample will have a smaller error than any other single measurement and will be approximately equal in precision to the combined result from 19 different measurements made by the four LEP experiments. The amount of data with the endcap CRID operating will be modest, but we should be able to observe Cherenkov rings and demonstrate the functionality of the system.

The SLD collaboration and the SLAC management have plans to extend the operations of the SLD for several years, the goal being one million $Z^{\circ}$ 's with electron polarization above $65 \%$. A proposal is in preparation detailing the physics topics to be studied and the machine and detector performance that will make this goal achievable. We have made a very considerable investment in the SLD and the physics payoff is now becoming evident; the proposed extension of the program would lead to world-beating results in the measurement of electroweak parameters. Our Boston University group would devote one postdoc full-time to the SLD data-taking and analysis; graduate student John Coller will do his doctoral thesis on data from the SLD.

\section{GEM Activities}

For the past two years we have been working on the GEM Experiment for the SSC. We are one of the principal groups working on the endcap muon system. We, along with the Brookhaven National Laboratory group led by V. Polychronakos, have advocated the use of cathode strip chambers (CSC's) for the GEM muon system. This technology was adopted for the full GEM muon system and we have played a major role in the development and test of prototype chambers, in the specification of the GEM muon system based on CSC's, and in the preparation of the GEM Technical Design Report that was presented to the SSC PAC in May 1993. 
Support for this work has been provided by the TNRLC, as part of the GEM R\&D program, as well as by the DOE SSC Office. Typically, two Boston University engineers have been devoted full time to the development of CSC electronics and mechanics. We have been collaborating in this work with groups from Brookhaven National Laboratory, SUNY Stony Brook, Draper Laboratory, and the St. Petersburg Nuclear Physics Institute; other groups have been joining the CSC effort, including Michigan State University and MIT.

The layout of the GEM detector as presented in the TDR is shown in figure 3. Muon trajectories are measured in a 0.8 Tesla magnetic field outside the calorimeter. Muon momentum is determined by the sagitta method using measurements of muon position at the beginning, middle, and end of the particle's passage through the field region.

The CSC's provide the full functionality of the muon system. Each module will consist of six chambers (eight for the inner endcap modules). The wires, which run in the azimuthal direction, are ganged for measurement of the radial coordinate with approximately $2 \mathrm{~cm}$ resolution. With a maximum drift time of approximately $25 \mathrm{~ns}$ in each chamber, a module will provide timing resolution of approximately $4 \mathrm{~ns}$ - sufficient to tag the bunch crossing in which the interaction occurred. Interpolation of the induced charges on the cathode strips will determine the azimuthal position of the track with better than $75 \mu$ resolution per chamber. Readout of the strips will be done with on-board multiplexing, so the cable plant will be minimal. Fast signals off the strips will be used to generate a first level trigger. The CSC modules overlap to provide complete coverage in azimuth. In the endcap region between approxinuately 9.8 and 27.7 degrees in polar angle, the modules overlap in $\theta$ as well, providing full coverage, while in the barrel the chamber layout and the projective alignment system lead to gaps in coverage.

Test chambers and readout electronics have been constructed at Boston University to study the time response, gain performance, and position resolution. Readout circuits using the Amplex chip have been developed; equivalent noise charge of less than 2,000 electrons and channel-to-channel uniformity of better than $2 \%$ have been demonstrated. We have tested a four-chamber system at the RD5 muon test beam at CERN. Despite some high voltage problems and a less-than-optimal calibration system, we succeeded in demonstrating spatial resolution better than 80 microns. Figure 4 shows the design of the chamber. Figure 5 plots the residuals of the position measurement in cne chamber relative 
to the line fit to the hits in the other three chambers. After correcting for the errors of the line fit, the single layer resolution was 79 microns.

The CSC anode wires measure the non-bend coordinate and determine the timing of the event. The anode readout chain has been designed at Boston University and is being prototyped here for the next generation of CSC's now under construction in a joint effort of the entire GEM muon group. We will provide up to 500 channels of anode readout, incorporating preamplifiers and shaping amplifiers made at St. Petersburg Nuclear Physics Institute along with discrimination and trigger circuits that we are developing. Printed circuit boards are now being fabricated, and the full system will be ready for tests in the Fall.

We have also been pursuing tests of the mechanical properties of the G10-honeycomb laminates that will be used in the CSC construction. We have made a test chamber in which humidity can be controlled and in which the temperature and humidity can be continuously monitored. We have measured a thermal expansion coefficient for the laminates of $1.6 \times 10^{-5} /{ }^{\circ} \mathrm{C}$, in agreement with bulk properties of the G10. Indications of humidity response are being studied.

\section{Activities of the Coming Year}

Work for the coming year will concentrate on design and construction of a full-scale CSC implementing the features described in the Technical Design Report. Boston University is responsible for the anode readout electronics, as noted above. We will also continue our work on readout integration, high voltage systems, and integration of alignment and support features. Working with our collaborators in the GEM muon system, we will finalize the CSC chamber design, optimize it for production, and begin to develop production plans for the full complement of barrel and endcap CSC's.

Tests of CSC performance in magnetic fields will be a crucial validation of the technology. We have designed and are constructing a chamber in which the orientation of the anode wir's relative to the cathode strips can be changed, allowing us to test magnetic field effects and Lorentz drift compensation while minimizing systematic errors. This chamber will be tested in a magnetic field during the coming year. We will also continue our tests at RD5, using a prototype chamber built at BNL to study systematics of chamber operation. 


\section{The Beijing Spectrometer (BES)}

It is likely that the SLD will terminate data-taking in a few years, and we have spent part of the past year planning for our next project. Our contributions to the SSC are successful and we plan to pursue them (assuming the project goes forward!). To keep a balanced program during the years until the SSC operates we want to be involved in a project which will provide world class physics for at least the next five years. Our choice is to pursue the opportunities in $e^{+} e^{-}$physics at the Beijing Electron Positron Collider (BEPC).

$\mathrm{BEPC}$ is a $2.0-2.8 \mathrm{GeV} /$ bearn $\mathrm{e}^{+} \mathrm{e}^{-}$collider. It was designed with substantial assistance from accelerator physicists at SLAC and was based upon the very successful SPEAR storage ring. Experiments at SPEAR provided an impressive range of physics results for over a decade. The Beijing collider will be the source for the next generation of experiments in the very fruitful energy region around the $\mathrm{J} / \psi, \tau$, and charm thresholds. It is expected to achieve a maximum value of $1.7 \times 10^{31} \mathrm{~cm}^{-2} \mathrm{sec}^{-1}$ at an energy of $2.8 \mathrm{GeV}$ per beam. It will have a luminosity 4 to 5 times that of SPEAR, and it will be scheduled for dedicated High Energy Physics runs 2 to 3 times as much each year as SPEAR was able to manage.

A collaboration of Chinese physicists was formed to build a detector, the Beijing Spectrometer (BES), which was nearly a direct copy of the Mark III detector at SPEAR. After several years of running, it became clear that collaboration with U.S. physicists could provide needed expertise in analysis. A group of U.S. institutions have joined the project, with Walter Toki of CSU as spokesman. We will participate in operating the experiment and analyzing the data, and we also propose to take part in the upgrades needed to maintain and improve the performance of the detector.

One of the first important projects at BEPC has been to do a careful scan across the $\tau$ threshold to determine the $\tau$ mass more precisely. This measurement is important due to the dependence of the decay width on the mass:

$$
\Gamma(\tau \rightarrow \mu \nu \bar{\nu})=G_{\tau}^{5} m_{\tau}^{5} / 192 \pi^{3}
$$

A similar expression exists for $\mu$ decay. By using the measured $\tau$ lifetime and branching ratio one can test whether the weak coupling constants $G_{\tau}$ and $G_{\mu}$ are equal. With current values there is a $2 \sigma$ difference. The suspicion has been that the $m_{\tau}$ is incorrect. The result 
from the BES scan, as published last Fall but with a smaller error die to higher statistics from including additional $\tau$ decay channels, is $\mathrm{m}_{\tau}=1776.9 \pm 0.2 \pm 0.2 \mathrm{MeV}, 7.2 \mathrm{MeV}$ lower and considerably more precise than the world average of $1784.1(+2.7,-3.6) \mathrm{MeV}$. Argus and CLEO also presented new tau mass determinations, in agreement with the BES result but with significantly larger errors.

The recently completed run of the BES experiment focused on data-taking at $4.03 \mathrm{GeV}$ in the center of mass to study the $D_{S}$. Measurements of the $D_{S}$ mass and some decay modes will be presented at this summer's conferences. While significant data were collected, both the accelerator and the detector had occasionally difficulties that highlighted the necessity for refurbishing and upgrading the hardware systems.

In preparation for the upgrade we have done some preliminary studies on the drift chamber end plates and on the feedthroughs to support the drift chamber sense wires in the end plates. The Boston University Scientific Instrument Facility has recently acquired a Giddings and Lewss 4" horizontal boring mill, a very large and extremely precise CNC machine that has the capacity to machine and drill the 90 " diameter end plates to the required $0.002 "$ tolerances. We have tested some machining techniques on small scale to plan the end plate fabrication and provided input to the upgrade planning. We have also fabricated a few sample pieces of the sense wire feedthrough design. This feedthrough has a 100 micron diameter hole that must be concentric within 50 microns with the $4 \mathrm{~mm}$ outer diameter. These tolerances are outside the capability of molding, however we were able to meet them by machining the pieces in our CNC watchmaker's lathe. This was a demonstration of feasibility, but it may be the best means of producing the approximately 7,000 sense wire feedthroughs needed for the full chamber.

\section{Planned Activities}

We are viewing the BES experiment as a long range activity. While we have participated to a limited extent in software development, our present involvement focuses on the preparation of the upgraded detector. The mechanical shop facilities at Boston University have a unique capacity that will enable us to make major contributions to the hardware upgrades to BES. The major task will be to machine the end plates and supporting structure for the replacement of the main drift chamber (MDC) of BES. The MDC is demonstrating some of the classic symptoms of drift chamber aging, such as gain losses and increased noise. Inner and outer layers have also experienced HV problems that require operation 
at reduced voltage. The replacement chamber has been redesigned to have better field uniformity for the first and last layers, and it will have a better symmetry to the drift cells that should result in improved performance.

A proposal for the upgrade program and the role of the U.S. participants was submitted to the D.O.E. last Spring. The spirit in which we at Boston University are undertaking these upgrade tasks is to identify areas where we can make a unique contribution to the project through our superior infrastructure and facilities. The work on the MDC proposed to be done at Boston University is the critical path item in the upgrade. In order to maintain the required schedule, we have already purchased the aluminum blanks for the new end plates and are preparing to proceed with the machining. The upgrade program gives us an opportunity to contribute to the future productivity of this experiment and to assure that the data will be of the high quality needed to match the high statistics.

\section{Budget Discussion}

The budget page for the CB group presents the base funding request for the year starting February 1, 1994. The bottom line is equal to the present total for Task A and Task L, where half the salary of J. Shank was the sole operations item. We want to eliminate operations items from Task $\mathrm{L}$ and leave it as the equipment task for SSC muon detector work. We have requested equipment funds under Task A for the BES upgrade in keeping with the Boston University responsibilities detailed in the BES upgrade proposal. 


\section{Recent Publications by Members of the CB Group}

1. Measurement of the Mass of the Tau Lepton. J. Z. Bai et. al. (BES Collaboration), Phys. Rev. Lett. 69, 3021 (1992).

2. First measurement of the left-right cross-section asymmetry in $\mathrm{Z}$ boson production in $\mathrm{e}^{+} \mathrm{e}^{-}$collisions. K. Abe et. al. (SLD Collaboration), Phys. Rev. Lett. 70, 2515 (1993).

3. Measurement of $R_{b}=\Gamma(b b) / \Gamma(h a d)$ at SLD. K. Abe et. al. (SLD Collaboration), SLAC-PUB-6189, May 1993, to be submitted to Phys. Rev. Lett.

4. Measurements of Jet Rates and $\alpha_{s}$ at the Z Resonance. K. Abe et. al. (SLD Collaboration), SLAC-PUB-6189, May 1993, to be submitted to Phys. Rev. Lett.

5. The GEM Muon System Based on Cathode Strip Chambers. J. S. Whitaker et. al., GEM TN-92-199, September 1992.

6. GEM Technical Design Report. GEM Collaboration, GEM TN-93-262 and SSCL-SR1219, April 1993.

7. CSC Tests at RD5. J. Shank et. al., GEM TN-93-402, April 1993. 

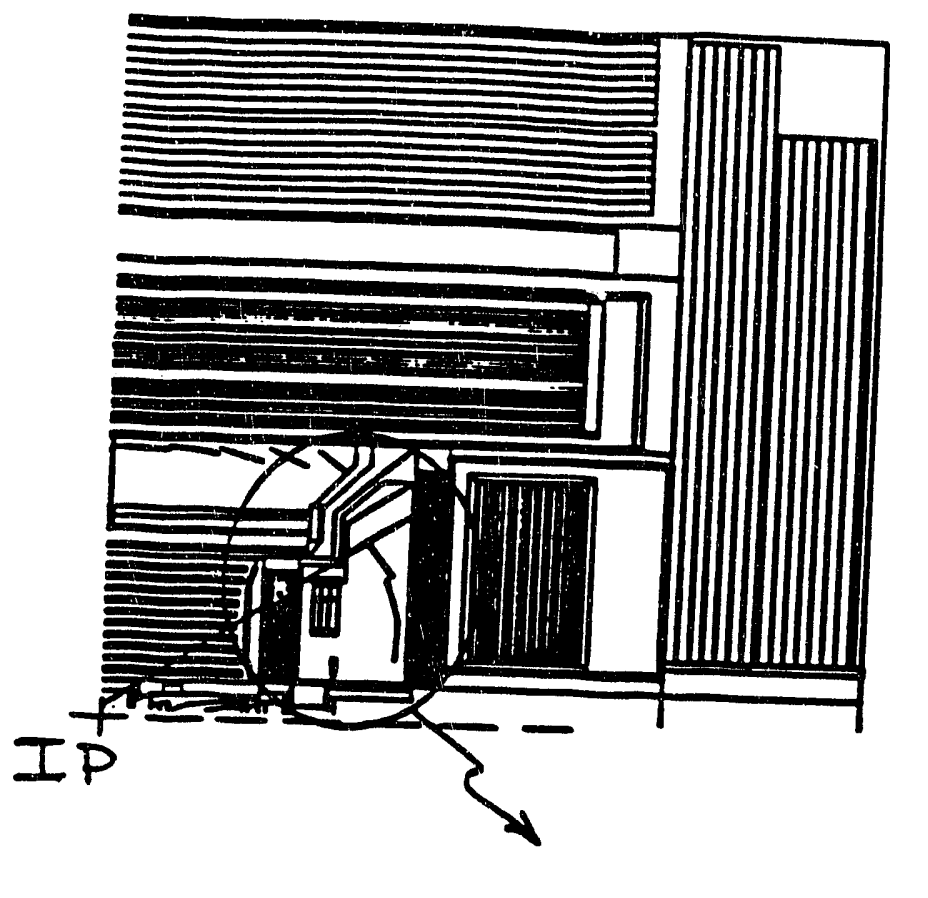


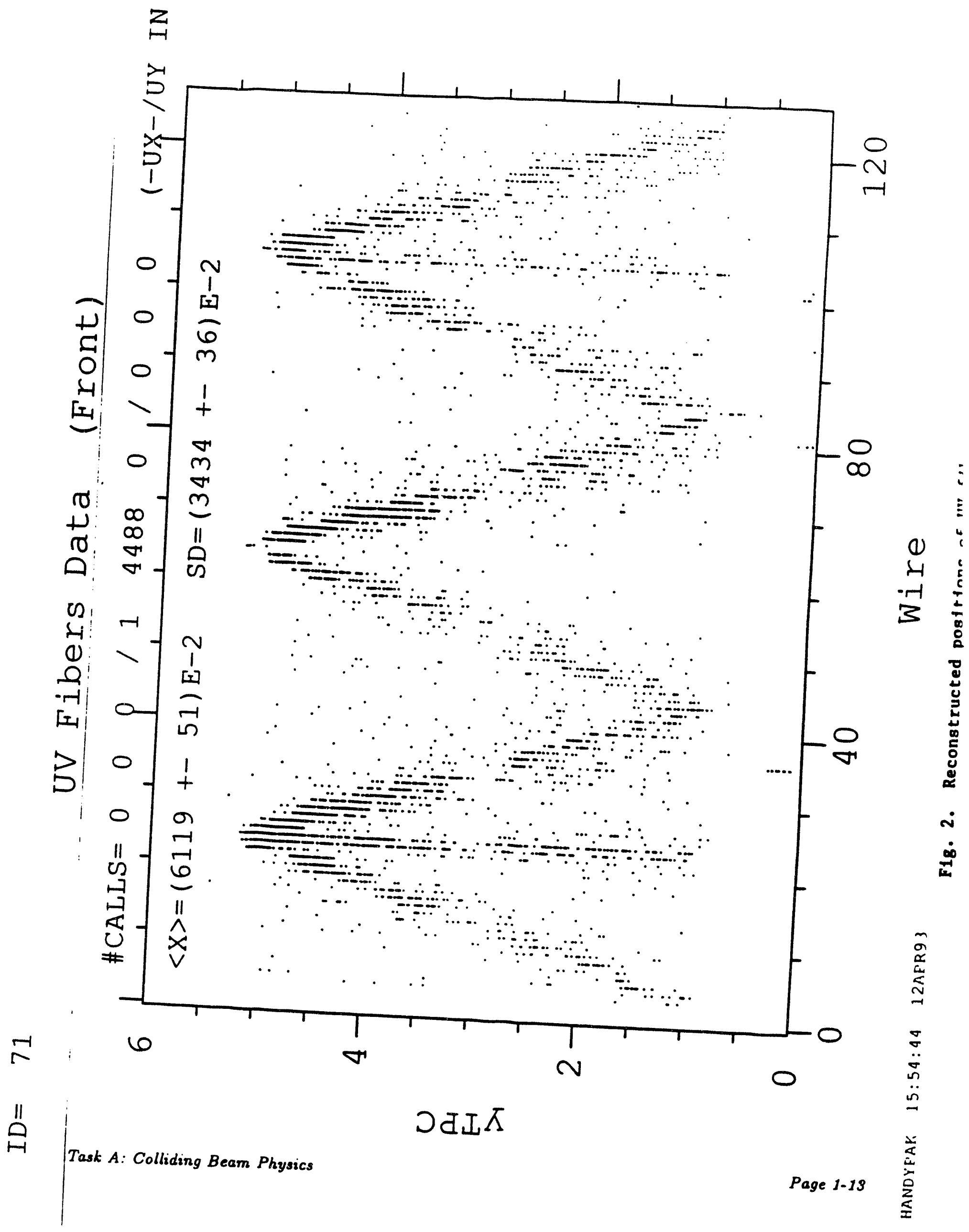



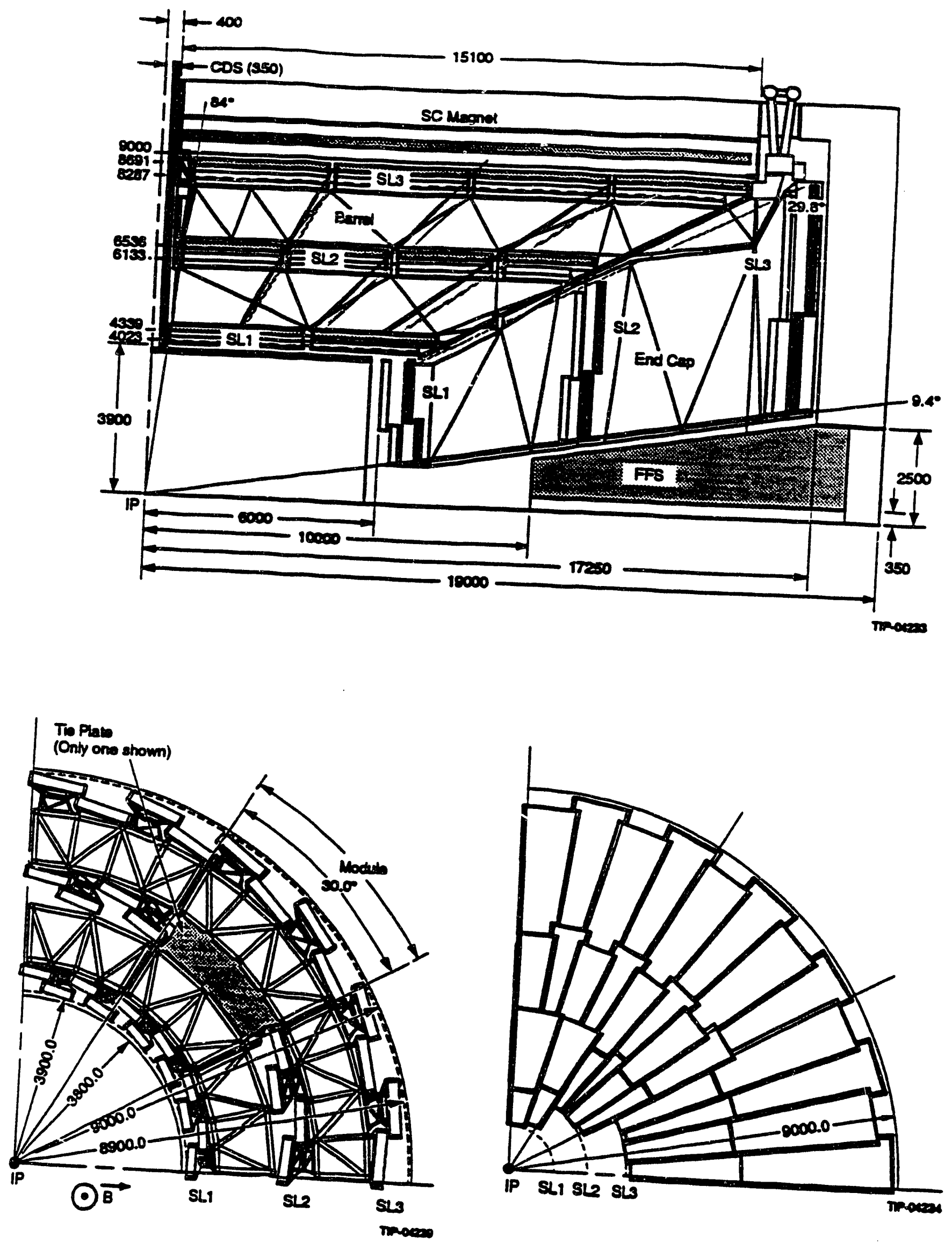

Page 1.14 Fig. 3. Layout of the GEM muon system 


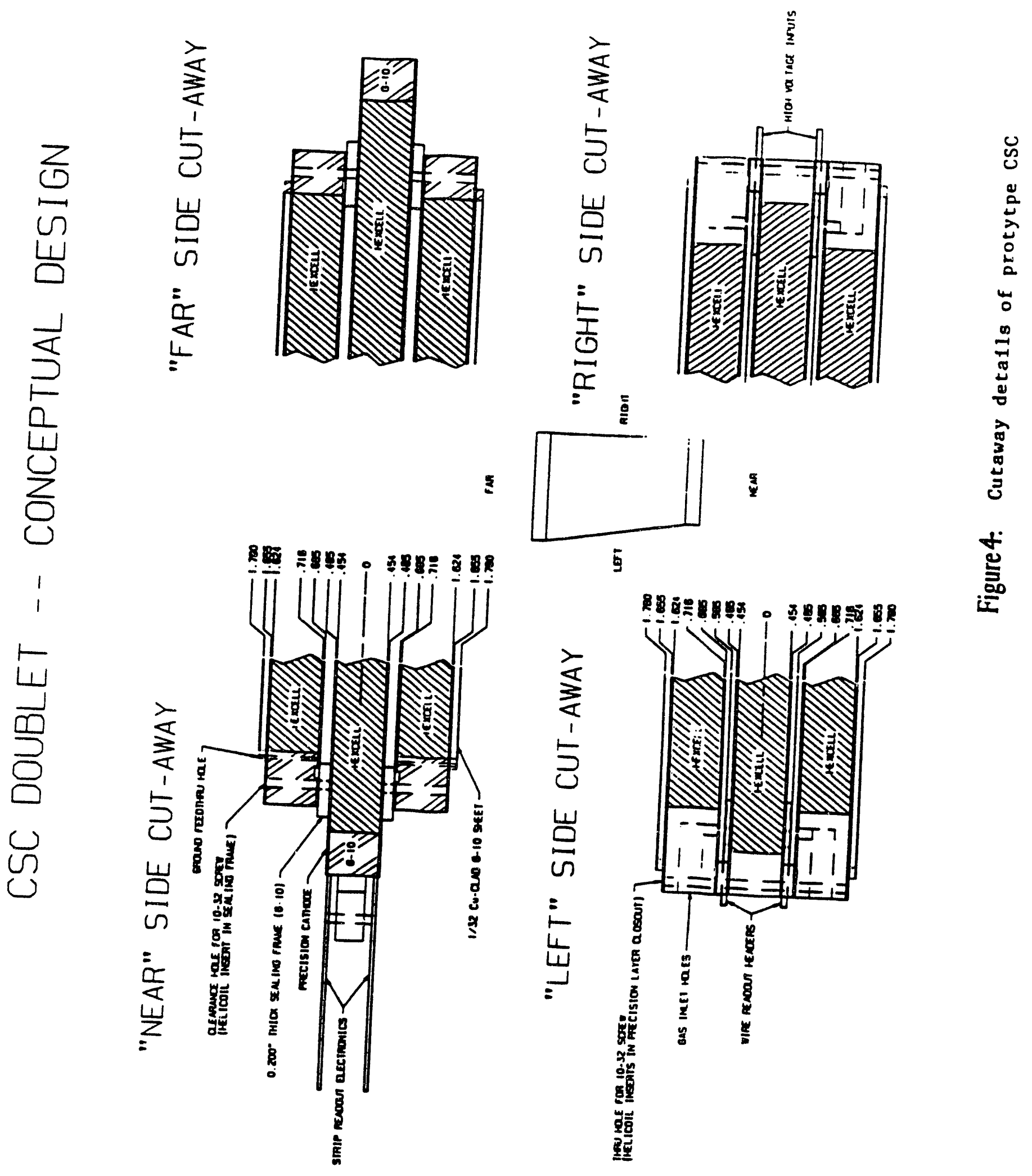

Task A: Colliding Beam Physics 
GEM CSC RDS Data.

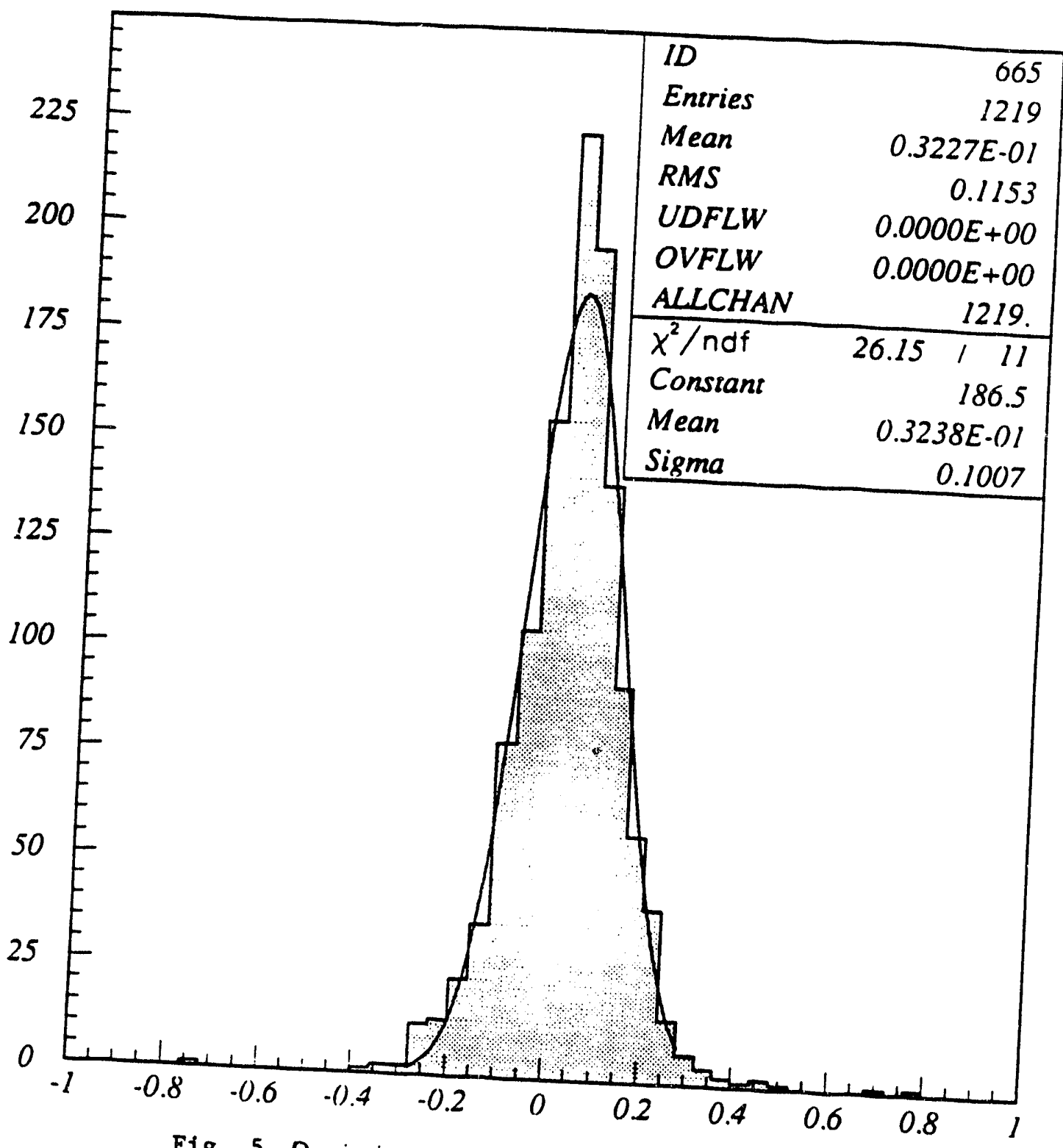

Fig. 5. Deviation of hits in layer 2 from fitted line $(\mathrm{mm})$. 


\section{TASK C: PARTICLE ASTROPHYSICS GROUP}

Faculty:

Research Faculty:

Research Associate:

Graduate Students:

Technician:

Collaborating Institutions

MACRO Experiment: Bari, Bartol, Bologna, Caltech, Drexel, Frascati, Indiana, L'Aquila, Lecce, Michigan, Napoli, Pisa, Potenza, Roma, Sandia, Texas A \& M, Torino

\section{Introduction:}

The Particle Astrophysics Group has research involvement with t'se MACRO experiment which dates to its inception in 1984-85. The building of this experiment has proceeded steadily over the past few years in a staged fashion with parts of the detector being brought online for data taking as others were under construction. The MACRO (Monopole, Astrophysics, and Cosmic Ray Observatory) experiment is located at the Gran Sasso Underground Laboratory near L'Aquila, Italy. It consists of a large area detector dedicated to a search for GUT monopoles and studies of muon and neutrino astrophysics. The MACRO detector is modular and consists of 6 upper and 6 lower supermodules each measuring 12 $\mathrm{m} \times 12 \mathrm{~m} \times 5 \mathrm{~m}$. The lower supermodules consist of 10 layers of streamer tube tracking separated by $32 \mathrm{~cm}$ of crushed rock absorber. This inner tracking section is surrounded by tanks of liquid scintillator viewed by 8-inch photomultiplier tubes (PMTs); the scintillator system is the primary responsibility of the U.S. collaborating institutions. The scintillator system performs muon timing and triggering in addition to its main requirement of sensitivity to slow ionizing particles. The upper supermodules (attico) form a hollow structure with more tanks of liquid scintillator sandwiched with streamer tubes. This cavity houses 
the readout electronics, and allows for future upgrades such as transition radiation detectors, a solenoidal magnet or additional absorber to identify stopping particles. As a final redundancy for monopoles and other heavily ionizing particles, plates of CR39 and Lexan plastic track etch detector cover the central plane and one face of the detector.

The first supermodule of MACRO has been running since February, 1989. The lower 6 supermodules commenced data taking in November 1992 and have recently completed an uninterrupted 6 month run. The attico is mechanically complete and is currently being instrumented. The full MACRO detector is scheduled to be taking data starting in September 1993.

\section{Physics with MACRO}

The idea of the existence of magnetic monopoles dates back to Maxwell's formulation of electricity and magnetism. Then it was seen as a way to symmetrize the Maxwell equations. In 1931, Dirac showed that the existence of a monopole would explain the quantization of charge. More recently, 't Hooft and Polyakov showed that monopoles are stable solutions of any non-Abelian field theory where a larger group undergoes spontaneous symmetry breaking to produce a $U(1)$ group. In the minimal SU(5) theory, monopoles would have a mass of $\sim 10^{16} \mathrm{GeV} / \mathrm{c}^{2}$ though other theories predict ralues that span many orders of magnitude around this value. Such monopoles would be expected to be produced in the early universe, and distributed throughout the universe.

Astrophysical considerations place limits on monopole abundances and velocities in the universe. The Parker limit is based on the observed galactic magnetic field, which would be destroyed in the presence of a large monopole flux. For minimal SU(5) and monopole velocities of $\sim 10^{-3} c$, the upper bound on the flux is $\sim 5 \times 10^{-15} \mathrm{~cm}^{-2} \mathrm{sr}^{-1} \mathrm{~s}^{-1}$. Gravitational and magnetic interactions suggest monopole velocities as low as $10^{-4} \mathrm{c}$. Theoretical and astrophysical considerations suggest an extremely small flux of super-heavy relativeiy slow monopoles. A search for such monopoles, as well as other exotica such as strange quark matter, requires a very large area detector capable of detecting slowly moving, highly penetrating particles.

The large acceptance $\left(\sim 800 \mathrm{~m}^{2} \mathrm{sr}^{-1}\right)$ and high resolution tracking of the MACRO detector allows detailed studies of muons from primary cosmic ray interactions in the upper atmosphere. MACRO will provide an enormous data sample of underground muons 
( $\sim 10^{8}$ single muons and $\sim 10^{6}$ multiple muons per year). The single muon data allow for searches of cosmic ray anisotropies, time variations, and high energy point sources as well as study of the cosmic ray spectrum. The study of the rates and muon separation in multiple muon events will provide insight to both the primary cosmic ray composition and their interaction with nuclei in the upper atmosphere. In addition, MACRO is collaborating with an extensive air shower array located on the top of Gran Sasso (EAS-TOP), which will provide a unique ability to study coincident events passing through both detectors.

Upward-going muons originate from neutrino interactions in the rock below MACRO. These atmospheric neutrinos come from pion and kaon decays in cosmic ray showers in the opposite hemisphere from the detector. It is of great interest to account in detail for the number and energy spectrum of atmospheric neutrinos because this is sensitive to flavor oscillation of massive neutrinos. In addition, upward-going muons can be used for searches for WIMPs accumulating in either the earth or the sun as well as for neutrino astronomy.

The energy released by a gravitational stellar collapse is mostly emitted as $\nu$ and $\bar{\nu}$ of all families during the neutron star cooling phase $(\sim 10 \mathrm{sec})$. The observation of SN1987A by the Kamiokande and IMB experiments largely confirmed the theory of supernova explosion and neutrino emission. Upon completion, MACRO will have a large active mass ( $\sim 600$ ton) allowing us to search for stellar collapse events. The detected reaction would be scattering: $\bar{\nu}+p \rightarrow n+e$ where the neutrino has an average energy around $10 \mathrm{MeV}$. The delayed gamma from neutron capture $(2.2 \mathrm{MeV} \sim 180 \mu \mathrm{sec}$ delayed) provides a unique secondary signal that can be detected with $\sim 25 \%$ efficiency assuming a $1.5 \mathrm{MeV}$ threshold. Scintillation detectors are more sensitive to this secondary signal due to their lower energy thresholds than the water Cherenkov detectors. 


\section{Status of the MACRO Detector}

The MACRO detector has been assembled in a staged fashion, which allowed us the opportunity to commission the first fraction of it for data taking while assembly continued on the rest. The first major stage was the completion of the first supermodule in February 1989. The data from the first one-twelfth of the final design has already provided for three Ph.D. theses (two from Boston University: D. Ficenec and D. Levin) and several publications and conference contributions (see Bibliography). Most importantly, it has provided working experience from which we can benefit as we assemble and commission the remainder of the detector.

The second major stage for MACRO was the completion of the lower 6 supermodules. The streamer tube tracking for supermodule 2 has been operational since May 1990. Several analyses have been performed using the tracking volume of supermodules 1 and 2 alone. The tracking for all six lower supermodules has been operational since July 1991, and provided the data for the Boston University thesis of R. Cormack. In parallel, the U.S. collaborators have commissioned the scintillator system for supermodules 2 through 6 . The lower 6 supermodules (tracking + scintillator) commenced full operation in November, 1992. The upper 6 supermodules of the attico are anticipated to be online by September 1993.

\section{Responsibilities and Future Activities of the BU Group}

In the MACRO detector, timing and energy loss information are provided by the signals from the PMTs viewing the scintillation counters frnm both ends. The BU group was responsible for providing all of the front-end components including the PMTs and associated hardware. This involved the evaluation of candidate PMTs, purchase, and certification of all PMTs $(\sim 1500)$ in the scintillator system. In addition we provided the cables to supply HV to the tube and to bring the signals to the readout electronics. We were also responsible for the development and production of the PMT base PC board, providing HV distribution to the dynodes and anode readout. The wide variety of physics being investigated by MACRO necessitates a number of electronics systems, each requiring an accurate copy of the PMT signal. The BU group designed a fanout module that takes 8 PMT signal inputs and produces 6 output copies of each signal. It is based on the Harris 
HA3-5002-5 buffer amplifier. Six supermodules of MACRO required 720 fanout modules. produced by the $\mathrm{BU}$ group.

Because MACRO is searching for extremely rare effects, it is essential to understand the behavior of the scintillator, PMTs and electronics. The Boston group supplied the calibration system for MACRO. This consists of two systems. The first is a system of lasers, computer controlled attenuators, and optical fibers to introduce precisely controlled amounts of light into the scintillation counters, providing calibrations of timing and saturation effects. The second is a system of LEDs, computer controlled pulse generators and relays, providing information about timing, PMT gain and trigger efficiencies. In the next section of this report we will describe the first measurement of slow monopole sensitivity using the LED system.

The $200 \mathrm{MHz}$ waveform digitizer (WFD) system is one of the last major electronics systems needed for the MACRO experiment. The purpose of this system is to record full wave forms at very high sampling rate ( $5 \mathrm{~ns}$ ) for the various types of signals expected. The WFDs are a key component in our search for slowly moving magnetic ruonopoles. Recognizing the urgent need for this critical circuit, the BU group initiated a design and development effort. A circuit was developed, tested in a CAMAC prototype, and is currently undergoing its final revisions and layout for VMEbus operation. The final system will be multiplexed 2:1 with 4 input channels per VME module. We describe this system in more detail in a subsequent section.

\section{Slow Monopole Sensitivity Mensurement using the LED Calibration System}

The validity of a search for exotic, and as yet undiscovered, particles is contingent upon two factors: a theoretical understanding of the interaction between particle and detector (i.e. energy loss as a function of various particle parameters), and an experimental understanding of the sensitivity of the detector and electronics to such an interaction. Much theoretical work has been done to understand the energy loss mechanisms in liquid scintillator of slow particles such as massive magnetic monopoles and strange quark matter. These have led to response curves of energy loss (measured in li - $^{-} \mathrm{t}$ yield per unit path length) as a function of particle velocity. Thus, MACRO needs to uriderstand its detection probability as a function of those same variables. This would give, for a given particle, the minimum velocity detectable by the MACRO experiment. 
As we have available no slow pariicle test beams, we use Light Emitting Diodes (LEDs) to generate known amounts of light over given pulse durations. Every MACRO tank end contains a mirror designed to collect scintillation light and focus it on the phototube photocathodes. On each mirror is muunted an LED. In the past, these LEDs were driven simultaneously by a single pulse generator. This allowed for only very crude simulations of slow particle energy deposition. No online simulations, consistent with a real particle passage, were possible. At Boston University, we have designed, built, and implemented a CAMAC controlled system that allows us to select, arbitrarily, only those LEDs we wish to fire and to fire each of them with any one of four completely independent pulses. A true slow particle passing through two planes of MACRO scintillator would generate four phototube signals, each with different pulse heights, widths, and delays. This new system allows us to simulate just that, at the most fundamental level possible without using actual particles. Simultaneously, we are testing phototube response, analog signal prosessing, trigger electronics, waveform recording, data storage, and analysis software. Thus, we can directly measure our sensitivity to slow particles taking directly into account every aspect. of the experiment from the phototube to the graduate student.

This system has been operational for the duration of the six-month run. The first and possibly most important piece of information it provided was that, for that run, our monopole sensitivity was limited not by the trigger or by background, but by the waveform digitizer system. Currently, we use LeCroy 2261 waveform digitizers with 16::1 multiplexing at each channel. The fan-ins employed in the multiplexing degrade phototube signals such that trains of separated single photoelectrons are not visible in the recorded waveforms. Thus, events that pass the slow monopole trigger threshold at minimal light levels appear as baseline waveforms in the data. This highlights the importance of bringing online the $200 \mathrm{MHz}$ waveiorm digitizers currently under development at Boston University.

Over the course of the six month run, LED "fake monopoles" were generated in specially designated runs each Tuesday. While the LED events are well documented as such both in the data and in offline summary files, the quality with which they mimic a true monopole signal is good enough that we are very hesitant to insert them into runs officially approved for data analysis. Many thousands of events, were generated. Pulse types were selected from over the entire interesting range in the $\mathrm{dL} / \mathrm{dx}$ vs $\beta$ phase space. These events are used to measure sensitivity and to search for potential systematic problems. 


\section{Waveform Digitizer Development}

One of the best handles on rare phenomena is the waveform of the signal from the PMTs in the scintillator system. Waveform digitization is essential for background rejection in searches for monopoles, and provides a powerful alternative to ADCs and TDCs for charge and time measurement. The narrow pulse width (15 ns) of the scintillation counters requires a system that has a high sample rate. Single photoelectrons are $4 \mathrm{mV}$ high, so we need a high gain amplifier to record small signals; however, the PMTs saturate at $10 \mathrm{~V}$, so we need pulse compression to record large signals. Zero suppression is desirable to keep the quantity of data manageable. These goals will be accomplished with a custom 200 $\mathrm{MHz}$ waveform digitizer (WFD) that has been developed at B.U. and is now entering the production stage.

Circuit operation is most easily separated into two parts, the high speed digitization process and VMEbus operations. For the most part these are independent in that VMEbus operations are not possible while digitization is occurring and vice-versa.

Digitization for a single channel begins with the four signal inputs being amplified and summed into a single output signal which is in the range 0 to $-2 \mathrm{~V}$ for the flash $\mathrm{A} / \mathrm{D}$ converter (FADC). Small signals are amplified by 10 to give $.8 \mathrm{mV} /$ bit resolution. Large signals are attenuated so that $-10 \mathrm{~V}$ input yields $-2 \mathrm{~V}$ at the FADC. The four input signals are also discriminated and the discriminator information is used to control zero suppression as well as provide a handle for distinguishing which of the four inputs contributed to the output pulse.

Each channel has an custom ASIC (Application Specific Integrated Circuit) designed at Boston U. Its function is to transform data appearing once every five nanoseconds from the FADC into parallel data which can be stored in RAM once every 20 nanoseconds. To do this, the sample and discriminator data are sequentially shifted through a series of four registers at a $200 \mathrm{MHz}$ rate. Thus, at any instant of time, registers in the ASIC contain the four most recent data samples and their associated threshold conditions.

If digitization is enabled, then all of the threshold bits for the four most recent samples are examined for non-zero values. Any non-zero bit initiates a sequence of operations via control logic. The first operation performed by this logic is the parallel strobing of the four sample values, the four sets of corresponding threshold bits and an internal sixteen-bit time word into eight eight-bit latches. The data in these latches remains stable for the 
next 20 nanoseconds, during which time it is transferred to a second set of latches and finally stored in appropriate RAM locations. The RAM address, also generated by the ASIC, is sent to external registers at this time. A shift register within the ASIC provides the signals for strobing the data and address information into these external registers and for generating the appropriate write pulses to the RAMs.

The VMEbus function is rather complex. Some of this comes from the need to have block transfer capability (for rapid readout of signals). Other complexity comes from allowing VMEbus to perform both write and read operations on the RAM in order to allow for memory testing.

The VMEbus addresses the RAM of each four channel circuit as a block of 256k bytes requiring 18 bits of address, the upper two bits used to select one of the four channels. A five bit switch allows the address of each waveform digitizer to be moved in blocks of $256 \mathrm{k}$ locations. This switch is intended to be used to set the block address to correspond to the slot number in the crate. An additional 9 bit switch allows the overall address to be moved in steps of $8 \mathrm{M}$ bytes over a range of $4000 \mathrm{M}$, the full 32 bit address range of VMEbus. All types of VMEbus data access are allowed, that is, single, double, triple, or quad byte operations. For block transfer operations, up to 256 bytes may be read or written at a time, but using only the lower eight address bits. The details of VMEbus operation involve the interaction of several control signals with rather stringent timing constraints.

The first prototype PC board underwent benchtop tests in early 1992 and was tested at the detector at Gran Sasso Laboratory in August 1992. Several design modifications were incorporated and the production prototype is currently being tested at Boston and will be tested at Gran Sasso in August 1993. If all goes well, production will begin immediately, to be completed by the end of 1993.

\section{Data Analysis at $B U$}

In addition to analysis with data from the the first stages of the MACRO detector, the Boston University group is continuing its analysis program with the recent data sample acquired from November 1992 to May 1993. This data consists of six complete supermodules of tracking and scintillation detector. 


\section{Atmospheric Neutrinos}

Because of their apparent stability and small probability for interaction, neutrinos can provide a window into physics that cannot be explored in any other manner. Cosmic rays interact with air nuclei in the atmosphere producing showers of particles. Among the particles generated are pions and kaons. The dominant decay modes of these short-lived particles produce muons and neutrinos. The muons generated here will decay to produce additional neutrinos. The neutrinos produced in these decay chains of the shower particles are considered atmospheric neutrinos.

In underground experiments, depending upon the phenomena one wishes to explore, these neutrinos can serve as either background or source. Searches for astrophysical sources, dark matter and proton decay see the atmospheric neutrinos as a background that must be well known so that it may be distinguished from a possible signal. Searches for neutrino oscillations, on the other hand, treat these neutrinos as a source flux that must be studied for variations from the expected signal. In either case, a good understanding of the characteristics of the neutrino flux must exist in order to interpret the results.

In MACRO, the atmospheric neutrinos cannot be observed directly. Instead, upwardgoing muons, the muons that are produced when neutrinos interact in the rock below the detector are detected. The flux of these atmospheric neutrino-induced muons at the level of the detector is given by:

$$
\frac{d N_{\mu}}{d E_{\mu}}=N_{A} \int_{E_{\mu}}^{\infty} d E_{\nu} \int_{0}^{\infty} d X \int_{E_{\mu}}^{E_{\nu}} d E_{\mu}^{\prime} g\left(X, E_{\mu}, E_{\mu}^{\prime}\right) \frac{d \sigma}{d E_{\mu}^{\prime}} \frac{d N_{\nu}}{d E_{\nu}}
$$

In this equation, $N_{A}$ is Avagadro's number, $d \sigma / d E_{\mu}$ is the cross section per nucleon for the process,

$$
\nu_{\mu}+N \rightarrow \mu+X
$$

and $d N_{\nu} / d E_{\nu}$ is the differential neutrino flux at zenith angle $\theta$. The term $g\left(X, E_{\mu}, E_{\mu}^{\prime}\right)$ is the probability that a muon of energy $E_{\mu}^{\prime}$ will have energy $E_{\mu}$ once it has traveled a distance $X$, and describes the energy loss of the muon in the rock.

GMACRO, a GEANT based simulation of MACRO, has been created for testing the detector response. This program accounts for all elements, active and passive, of the detector. Using GMACRO, with a variety of muon flux calculations, the signal of upward-going atmospheric-neutrino induced muons expected in MACRO due to has been studied. Approximately 200 of these muons per year are expected within the lower 6 supermodules of 
MACRO. In the future, when a large sample of upward-going muons has been found, comparisons of the angular distribution and overall rate of detected upward muons with those predicted by Monte Carlo will be performed. Based upon these comparisons, statements as to which, if any, flux model is favored by our data will be made.

Another way in which atmospheric neutrinos can be studied is through muons that stop in the detector. Observation of the stopping upward-going muons will allow a different region of the atmospheric neutrino spectrum to be examined. This analysis, when used in conjunction with the through-going muons can correct for ambiguities that exist in the neutrino flux and cross section used in the calculation of the neutrino-induced muon flux. As an example, the overall normalization of the atmospheric neutrino flux has been said to have an uncertainty of $20 \%$. If the ratio of stopping to through-going upward muons is used, the result is less dependent on this overall normalization. Monte Carlo studies have shown that approximately 50 upward-going stopping muons can be expected with one year of running with the lower 6 supermodules of MACRO. As with the through-going muons, by examining their overall rate and angular distribution, the stopping muons will help determine the most likely atmospheric neutrino model.

The inclusion of the attico will increase the rate of upward-going muons detected in MACRO. With the addition of the attico, the signature of stopping, upward-going muons would be an upward-going muon in the lower part of the detector which failed to exit through an attico plane. Also, depending upon the designs for the interior of the attico (open space or rock absorber), even more information can be gained from the stopping muons. As has been said before, the study of the stopping muons is important because it explores a different part of the atmospheric neutrino spectrum. Also, one of the methods that has been employed for searching for neutrino oscillations is to examine the ratio of stopping to through-going upward-going muons.

\section{Cosmic Ray Composition}

Cosmic rays are the only available samples of matter from beyond the solar system, and the origin of these ionized nuclei remains a mystery. Their composition reflects the nature of mechanisms that accelerate them to energies of $E \sim 10^{11} \mathrm{GeV}$ and the interactions that they undergo while traveling the galaxy. The "knee" of the cosmic ray energy spectrum at $\mathrm{E} \sim 10^{6} \mathrm{GeV}$ is the feature which theories of cosmic ray origin and propagation seek to explain. Competing theories differ in their predictions of the chemical composition at 
high energy. The primary flux at energy $E>\sim 10^{6} \mathrm{GeV}$ is expected to be dominated by nuclei of either the highest charge (iron) or the lowest charge (proton) and measurement of the primary composition in the region of the "knee" is an important to improve our understanding of our galaxy.

Experiments carried out by high altitude balloons and spacecraft have provided direct measurements of the cosmic ray flux at relatively low energies. The highest energy measurements approach the energy of the "knee," but they suffer from poor statistics due to the low particle flux and limited flight duration. Fortunately large ground based experiments may probe the composition above the "knee".

A number of deep underground detectors have attempted to infer the high energy composition from observed rates of multiple muon events. The sensitivity to composition arises from the increase of expected muon multiplicity with increasing nuclear mass. Measurements were performed with detectors having a typical size of $\sim 10 \mathrm{~m}$, producing event samples ranging from $10^{3}$ to $10^{4}$ events, and Monte Carlo simulations using simple models of underground muon rates. Poor statistics at high multiplicity limited the ability of such experiments determine the high energy composition.

MACRO is the largest area deep underground muon detector in the world and provides the means for detailed study of underground multiple muons. It has collected over $4 \times 10^{6}$ single muon and $2 \times 10^{5}$ multiple muon events containing muon pairs having $\sim 70 \mathrm{~m}$ separation. Analysis of the relative rates of muon multiplicity has been performed and compared to Monte Carlo predictions for composition models having different high energy behavior. Measurement of the separation of muon pairs provides a cross check of the interaction model used in the simulations.

MACRO provided the first test of the parameterizations of Forti et al. for muon separations up to $72 \mathrm{~m}$. The parameterizations were developed from results of simulations carried out with the HEMAS program for air shower simulation and are widely used by underground muon detectors. Underground muon events were generated for two composition models having values of $\langle\ln (A)>$ of $\sim 0$ (light composition) and $\sim 4$ (heavy composition). The observed multiplicity distribution is similar to that resulting from the simulation of the light composition model. However, muons were observed to have a greater separation than predicted by Monte Carlo. The result of the multiplicity analysis is similar to those from other deep underground detectors, but the separation distribution is the only mea- 
surement carried out beyond $\sim 20 \mathrm{~m}$. The deficiencies of the parameterizations prompted work using full shower simulation in the framework of HEMAS.

Full shower simulation allowed a number of improvements in the simulation of underground muon events. A more realistic method of nuclear fragmentation replaced the superposition model used to generate the parameterized muon distributions, and traching of individual particles allowed more realistic propagation of the muons through the overburden. However, simulation of complete air showers and subsequent propagation of muons require far more processor time. A new method of producing predictions for a given composition model was developed to be able to study various compositions in a reasonable period of time.

Completion of the work showed that the observed multiplicity distributions prefer Monte Carlo predictions based on the light composition model for primary energies up to $E \sim 10^{4} \mathrm{TeV}$. The preference of a light composition is not subject to changes in the simulation of the lateral spread of the showers, or in the depth of the detector. Variation of the flux above the knee, between $100 \%$ proton and $100 \%$ iron, indicated the maximum fraction of iron that would be consistent with observation to be $30 \%$. This work represents the first analysis based on complete shower simulation as opposed to the parameterizations of Forti et al.

A light high energy composition limits the possible theories of cosmic ray production and propagation which are consistent with the data. Theories that attribute the knee of the cosmic ray spectrum to acceleration mechanism inefficiencies or the onset of escape from the galaxy naturally result in the cosmic ray flux becoming predominantly heavy nuclei above energies of $\sim 10^{5} \mathrm{GeV}$ which is not consistent with the results of this work.

MACRO has compiled a large data set of multiple muons, and will continue to add to it for some time. Multiple muon analysis is no longer limited by low statistics of underground muon data, but more detailed work is needed to reduce the systematic errors in the measurement. Multiplicity analysis based on a complete detector simulation would provide a better measure of multiple muon rates, thus improving the limit for the maximum allowable fraction of iron. Using data from the scintillator system will also help to unravel event multiplicities. By measuring the energy loss in the scintillator, an estimate of the number of minimum ionizing particles passing through the tank can be made. New 200 $\mathrm{MHz}$ waveform digitizers may also be useful by providing information on the time structure 
of light arriving at the end of the tanks. Information from the scintillator system could be important for muon counting in very high multiplicity events where there is a significant probability of having many overlapping tracks.

Measurement of the energy of the primary cosmic ray on an event by event basis would improve the analysis by allowing composition studies to be carried out over smaller regions of the energy spectrum as opposed to the averaging over the entire spectrum that is currently done. The EAS-TOP experiment on top of Gran Sasso provides a measure of the electron component of cosmic ray showers. Preliminary tests of the GRACE experiment indicate that Ĉerenkov telescopes may also be useful in observing coincident surfaceunderground events. Work involving cooperation between these surface experiments should be continued. With the completion of the LVD detector in Hall A of the LNGS, it will become possible to observe portions of cosmic ray showers larger than MACRO alone can observe.

Multiplicity analyses are also subject to uncertainties in the Monte Carlo. The statistics of the pair separation distribution are not so good as the data and is determined by the contributions of the low energy primaries. Further simulations could be carried out for protons in the region of $50<E<200 \mathrm{TeV}$. More work is also required to determine the uncertainties in the interaction model used in HEMAS. Discussions with the authors indicate that the model used in HEMAS will over-predict the $p_{t}$ of secondary particles thus, over-predicting the lateral spread of muons. Work is proceeding on a new interaction model that better simulates secondary particle production.

Cosmic rays have played an important role in the development of particle physics. Now particle physics detectors are playing crucial roles in increasing our understanding of cosmic rays and, in turn, the nature of our galaxy. Though MACRO has logged only a small part of its expected life, it has already contributed much to cosmic ray physics, and it will continue to play an important role in the years to come.

\section{Magnetic Monopoles}

J.T. Hong has completed a search for slow-moving magnetic monopoles with the data collected over a period of two years from October 1989 to November 1991 using the first supermodule of the MACRO detector in his Ph.D. thesis.

Being 1/12 of the full MACRO detector, the first supermodule is surrounded on five sides by large tanks of liquid scintillator viewed by $20 \mathrm{~cm}$ hemispherical photomultiplier 


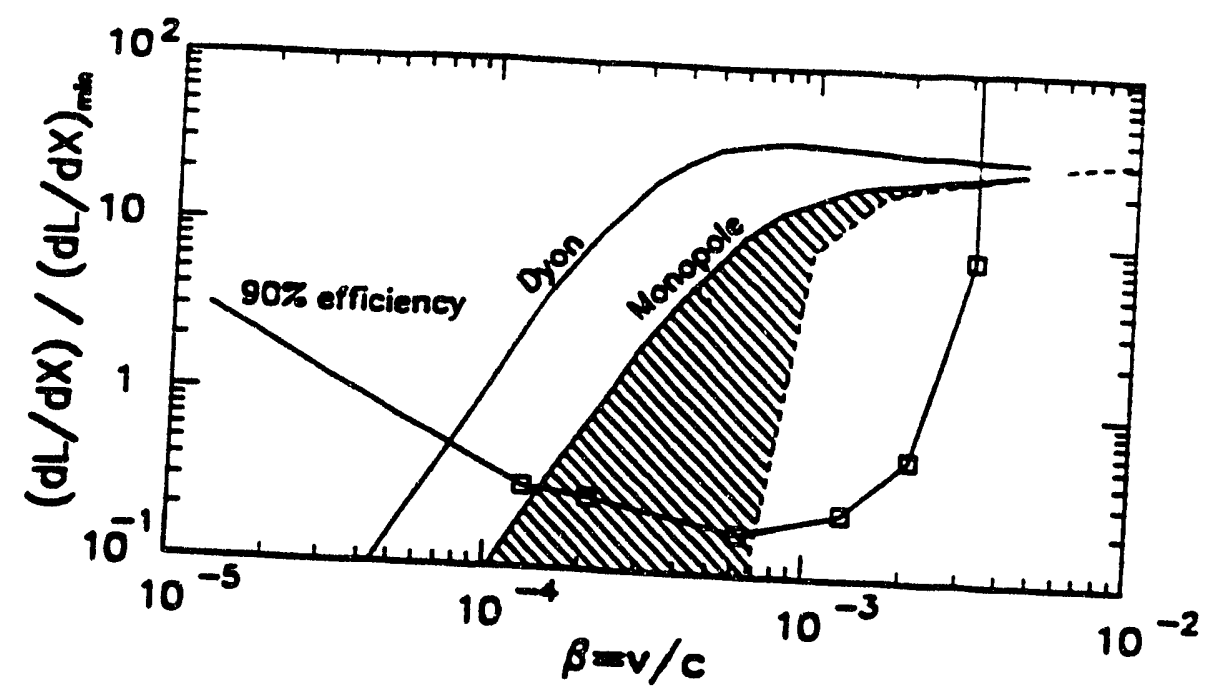

Figure 1: The measured slow monopole trigger sensitivity in horizontal tanks as compared with the expected light yields of monopoles measured curve to fire the slow a particle with light yield above the measured curve to fire the slow monopole trigger is greater than $90 \%$.

tubes. There are two types of tanks, horizontal and vertical. A specialized trigger circuit is used to identify the signature of a slow-moving particle crossing the thick scintillator layer (the slow monopole trigger). This trigger selects wide phototube pulses or long trains of single photoelectron pulses, rejecting any large but sharp pulses caused by muons or isolated background radioactivities. In the first supermodule, when a trigger occurred, the waveforms of the phototube response were recorded by two sets of waveform digitizers; the fast one was clocked at $50 \mathrm{MHz}$ and the input signal was attenuated to accommodate large pulse height, while the slow one was clocked at $20 \mathrm{MHz}$ and the input signal was amplified and stretched to resolve single photoelectron trains.

The sensitivity of this trigger to slow monopoles was measured by simulating the expected signals using LED pulses. The measured amount of light required to achieve $90 \%$ trigger efficiency (normalized to the light yield of minimum ionizing particles) as a function of the monopole velocity is shown in Figure 1.

Also shown are the expected light yields from bare monopoles and dyons (monopoles carrying a unit electric charge, or monopole-proton bound states). The shaded areas 
extend from the expected light yield based on the slow proton scintillation measurements by Ficenec et al. down to the most conservative estimate of light vield by Ahlen-Tarlé.

The data were collected from October 1989 to November 1991 and a detailed analysis was performed to search for monopoles. We required a minimum of two consecutive hours of good running conditions to insure stable operation of the detector. The integrated livetime for the slow monopole trigger analysis was 541 days, and there were 583,999 events with the slow monopole trigger present in at least one scintillator plane. After excluding events which also fired a fast two-plane muon trigger (time-of-flight $<1 \mu \mathrm{s}$, corresponding to $\beta>1.5 \times 10^{-2}$ ), we had 541,918 remaining events, the majority of which were caused by radioactivity pileups (many background radioactivity pulses accidentally occurring within a short time interval) in a single plane. We then selected events in which the slow monopole trigger was present in two separate planes within $600 \mu$ s (the longest time-of-flight for a $\beta \sim 10^{-4}$ monopole to cross the first supermodule). This left us with 573 events, each of which was examined and classified.

The majority of these events (388 events) were easily identified as electrical noise by the presence of bipolar oscillations in their recorded waveforms. Another 169 events had no feature in the waveform data; we interpreted these as noise on the trigger input. Another eight events had long pulse trains $(>4 \mu \mathrm{s})$ simultaneously present in every channel, inconsistent with the passage of particles. The only effect on monopole analysis of the above electrical noise events is a negligible reduction $\left(10^{-8}\right)$ in livetime because a monopole in accidental coincidence with one of the above noise events may be missed. Another two events are identified as muons because of their time-of-flight and pulse shapes; they escaped the fast muon veto because they occurred during a period when the fast muon trigger failed. Three other events had muon signals in one hit face and radioactivity pileups in the other hit face. Finally, the remaining three events had waveforms consisting of 4-8 narrow pulses in sequence and each pulse typically had a pulse height at least several times larger than the average single photoelectron pulse height. These three best candidates can be rejected based on the following three arguments: First, their waveforms are consistent with accidental coincidence of radioactivity pileups in both faces, but inconsistent with the passage of slow particles for which, as demonstrated by LED-simulated pulse trains, the photoelectrons should be randomly but uniformly distributed to produce a much smoother pulse than was observed. Second, no streamer tube signals were observed for these three 


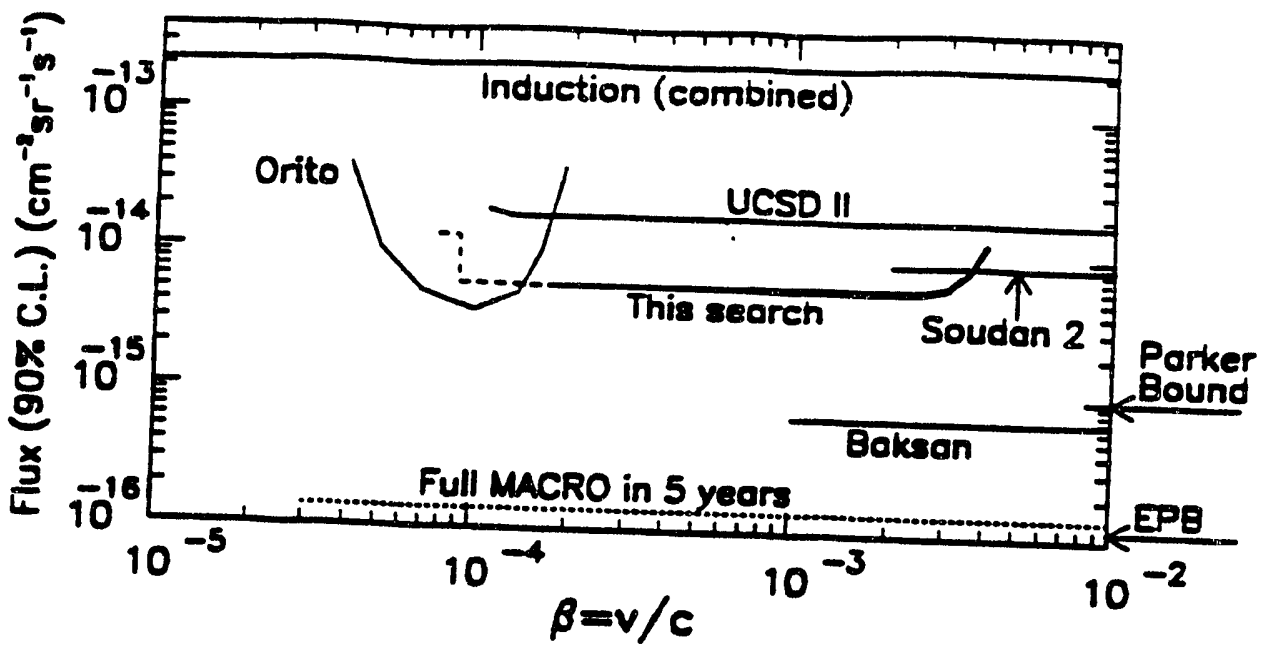

Figure 2: The upper limit on GUT monopole flux. See text for interpretation of this limit as a function of $\beta$.

signals. Third, trajectories clipping corners of scintillator tanks were required for these three events to reconcile the time-of-flight and the pulse train durations; since they use only a tiny fraction of the total acceptance, the probability for such corner-clipping events to occur is very small. The effect of these cuts on our monopole acceptance is negligible. Therefore, we concluded that no evidence was found for the passage through the apparatus of any slow-moving ionizing particles.

This negative search is used to establish an upper limit on the isotropic flux of GUT monopoles. The sensitive velocity range is determined by the trigger sensitivity versus light yield shown in Figure 1. The flux limit at $90 \%$ confidence level is shown in Figure 2.

We indicate with a bold solid line the most conservative velocity range that assumes the steep cutoff of the Ahlen-Tarlé model. The plain solid line indicates the additional sensitive velocity range derived from Ficenec et al.'s proton scintillation measurements. The dashed line extending below $\beta=10^{-4}$ assumes that the monopole is a dyon carrying a unit electric charge. This result increases below $\beta=9 \times 10^{-3}$ because only the horizontal tanks can be used in this region. Also shown is the anticipated limit reachable by the full MACRO detector after five years of operation, the astrophysical Parker bound, the recently-extended Parker bound (EPB) based on the survival of a small Galactic "seed" 
field, and the results from several previous searches: Induction (Combined). UCSD II ( $\left.\mathrm{He}-\mathrm{CH}_{4}\right)$, Soudan $2\left(\mathrm{Ar}-\mathrm{CO}_{2}\right)$, Baksan (scintillator), and Orito (CR-39).

\section{Summary of Publications by the BU Particle Astrophysics Group}

\section{Bibliography}

\section{Refereed Journals}

1. "Arrival Time Distributions of Very High Energy Cosmic Ray Muons in MACRO," S. Ahlen et al., Nucl. Phys. B370 (1992) 432.

2. "Study of the Ultrahigh-Energy Primary Cosmic Ray Composition with the MACRO Experiment," S. Ahlen et al., Phys. Rev. D46 (1992) 895.

3. "Search for Nuclearites Using the MACRO Detector," S. Ahlen et al., Phys. Rev. Lett. $\underline{69}$ (1992) 1860.

4. "Search for Muon Neutrino Oscillations with the Irvine-Michigan-Brookhaven Detector," R. Becker-Szendy et al., Phys. Rev. Lett. $\underline{69}$ (1992) 1010.

5. "Review of Particle Properties," Hernandez et al. (Particle Data Group), Phys. Rev. D45 (1992) 1.

7. "Search for Neutrino Bursts from Collapsing Stars with the MACRO Detector," S. Ahlen et al., Astroparticle Physics 1 (1992) 11.

8. "The Electron- and Muon-Neutrino Content of the Atmospheric Flux," R. BeckerSzendy et al., Phys. Rev. D46 (1992) 3720.

9. "Measurement of the Decoherence Function with the MACRO Detector at Gran Sasso," S. Ahlen et al., Phys. Rev. D46 (1992) 4836.

10. "Inclusive Jet Cross-Section in $\bar{p} p$ Collisions at $\sqrt{s}=1.8 \mathrm{TeV}, \mathrm{F}$. Abe et al., Phys. Rev. Lett. $\underline{68}$ (1992) 1104.

11. "The Topology of Three Jet Events in $\bar{p} p$ Collisions at $\sqrt{s}=1.8 \mathrm{TeV}$," F. Abe et al., Phys. Rev. D 45 (1992) 1448.

12. "A Lower Limit on the Top Quark Mass from Events with Two Leptons in $\bar{p} p$ Collisions at $\sqrt{s}=1.8 \mathrm{TeV}, "$ F. Abe et al., Phys. Rev. Lett. $\underline{68}$ (1992) 447.

13. "A Search for New Gauge Bosons in $\bar{p} p$ Collisions at $\sqrt{s}=1.8 \mathrm{TeV}, " \mathrm{~F}$. A be et al., Phys. Rev. Lett. $\underline{68}$ (1992) 1463. 
14. "Properties of Events with Large Total Transverse Energy Produced in $\bar{p} p$ Collisions at $\sqrt{s}=1.8 \mathrm{TeV}, "$ F. Abe et al., Phys. Rev. D $\underline{45}$ (1992) 2249.

15. "A Limit on the Top Quark Mass From $\bar{p} p$ Collisions at $\sqrt{s}=1.8 \mathrm{TeV}$," F. Abe et al., Phys. Rev. D $\underline{45}$ (1992) 3921.

16. "Measurement of the Isolated Prompt Photon Cross-Sections in $\bar{p} p$ Collisions at $\sqrt{s}=$ $1.8 \mathrm{TeV}, "$ F. Abe et al., Phys. Rev. Lett. $\underline{68}$ (1992) 2734.

17. "Measurement of the Ratio $\sigma B(W \rightarrow \tau \nu) / \sigma B(W \rightarrow e \nu)$ in $\bar{p} p$ Collisions at $\sqrt{s}=1.8$ TeV," F. Abe et al., Phys. Rev. Lett. 68 (1992) 3398.

18. "A Measurement of the $B$ Meson and $B$ Quark Cross-Sections at $\sqrt{s}=1.8 \mathrm{TeV}$ Using the Exclusive Decay $B^{ \pm} \rightarrow J / \psi K^{ \pm}$," F. Abe et al., Phys. Rev. Lett. 68 (1992) 3403.

19. "Lepton Asymmetry in $W$ Decays from $\bar{p} p$ Collisions at $\sqrt{s}=1.8 \mathrm{TeV}$ " $F$. Abe et al., FERMILAB-PUB-91-263-E; submitted to Phys. Rev. Lett.

20. "A Measurement of the Production and Muonic Decay Rate of $W$ and $Z$ Bosons in $\bar{p} p$ Collisions At $\sqrt{s}=1.8 \mathrm{TeV}, " F$. Abe et al., Phys.Rev.Lett. $\underline{69}$ (1992) 28 .

21. "Inclusive $J / \psi, \psi(2 s)$ and B-Quark Production in $\bar{p} p$ Collisions at $\sqrt{s}=1.8 \mathrm{TeV}, \mathrm{F}$. Abe et al., Phys. Rev. Lett. $\underline{69}$ (1992) 3704.

22. "Search for Squarks and Gluinos from $\bar{p} p$ Collisions at $\sqrt{s}=1.8 \mathrm{TeV}$," F. Abe et al., Phys. Rev. Lett. $\underline{69}$ (1992) 3439.

23. "Limit on the Rare Decay $W^{ \pm} \rightarrow \gamma \pi^{ \pm}$in $\bar{p} p$ Collisions at $\sqrt{s}=1.8 \mathrm{TeV}$," F. Abe et al., Phys. Rev. Lett. $\underline{69}$ (1992) 2160.

24. "The Dijet Angular Distribution in $\bar{p} p$ Collisions at $\sqrt{s}=1.8 \mathrm{TeV}$," F. Abe et al., Phys. Rev. Lett. $\underline{69}$ (1992) 2896.

25. "Limits on the Production of Massive Stable Charged Particles," F. Abe et al., Phys. Rev. D46 (1992) 1889.

26. "A Search for First Generation Leptoquarks in $\bar{p} p$ Collisions at $\sqrt{s}=1.8 \mathrm{TeV}$," $F$. Abe et al., FERMILAB-PUB-93-070-E, Apr. 1993. 20pp. Submitted to Phys. Rev. D.

27. "Measurement of the Bottom Quark Production Cross-Section Using Semileptonic Decay Electrons in $\bar{p} p$ Collisions at $\sqrt{s}=1.8 \mathrm{TeV}, " F$. Abe et al., FERMILAB-PUB93-091-E, Apr. 1993. 18pp. Submitted to Phys. Rev. Lett. 
28. "Measurement of Jet Multiplicity in W Events Produced in $\bar{p} p$ Collisions at $\sqrt{s}=$ $1.8 \mathrm{TeV}$, F. Abe et al., FERMILAB-PUB-93-063-E, Apr. 1993. 14pp. Submitted to Phys. Rev. Lett.

29. "The Center-of-Mass Angular Distribution of Prompt Photons Produced in $\bar{p} p$ Collisions at $\sqrt{s}=1.8 \mathrm{TeV}, "$ F. Abe et al., FERMILAB-PUB-93-032-E, Mar. 1993. 15pp. Submitted to Phys. Rev. Lett.

30. "Measurement of the Dijet, Mass Distribution in in $\bar{p} p$ Collisions at $\sqrt{s}=1.8 \mathrm{TeV}, " \mathrm{~F}$. Abe et al., FERMILAB-PUB-93-n17-E, Feb. 1993. 32pp. Submitted to Phys. Rev. D.

31. "Measurement of the Cross-Section Sx Production of Two Isolated Prompt Photons in in $\bar{p} p$ Collisions at $\sqrt{s}=1.8 \mathrm{TeV}, " F$. Abe et al., Phys. Rev. Lett. $\underline{70}$ (1993) 2232.

32. "Comparison of Jet Production in $\bar{p} p$ Collisions at $\sqrt{s}=546 \mathrm{GeV}$ and $1800 \mathrm{GeV}$," F. Abe et al., Phys. Rev. Lett. 70 (1993) 1376.

33. "Search for $\Lambda_{B} \rightarrow J / \psi \Lambda_{0}$ in $\bar{p} p$ Collisions at $\sqrt{s}=1.8 \mathrm{TeV}$," F. Abe et al., Phys. Rev. D47 (1993) 2639.

34. "A Measurement of Jet Shapes in $\bar{p} 0$ Collisions at $\sqrt{s}=1.8 \mathrm{TeV}$," F. Abe et al., Phys. Rev. Lett. 70 (1993) 713.

35. "The IMP-3 Detector: A Large Water Cherenkov Detector for Nucleon Decay and Neutrino Interactions," R. Becker-Szendy et al., Nucl. Inst. \& Meth. A324 (1993) 363.

36. "First Supermodule of the MACRO Detecior at Gran Sasso," S. Ahlen et al., Nucl. Inst. \& Meth. A324 (1993) 337.

Recent Invited Talks, Seminars, Colloquia of the BU Group

1. J. Stone, "Neutrino Physics with the MACRO Detector at Gran Sasso," invited talk, International Symposium on Neutrino Astrophysics, Takayama, Japan, Oct. 1992.

2. J. Stone, "Physics Where the Sun Doesn't Shine," colloquium, Louisiana State University, Baton Rouge, Feb. 1993.

3. J. Stone, "Physics with the Super-Kamiokande Detector," seminar, Bartol Research Institute, Newark, Mar. 1993.

4. J. Stone, "Proton Decay Experiments: Present Status and Future Prospects;" "Solar Neutrino Experiments: An Overview;" "Atmospheric Neutrino Measurements: An 
Overview," invited lectures, Third School on Non-Accelerator Particle Astrophysics. International Centre for Theoretical Physics, Trieste, Jul. 1993.

5. E. Kearns, "Recent Results from MACRO," seminar, Brookhaven National Laboratory, Sept. 1992.

6. J. Stone, "Physics with the Super-Kamiokande Detector," seminar, University of Maryland, Feb. 1993.

7. E. Kearns, "Search for Slow Ionizing Penetrating Particles with the MACRO Detector," invited talk, 7th Meeting of the APS Division of Particles and Fields, Batavia, Illinois, Nov. 1992.

8. R. Cormack, "Widely Separated Muons in the MACRO Detector," invited talk, 7th Meeting of the APS Division of Particles and Fields, Batavia, Illinois, Nov. 1992.

9. J. T. Hong, "Search for Slow-Moving GUT Magnetic Monopoles," contributed talk, The 1993 Joint April Meeting of the APS and the AAPT, Washington D.C., April 1993.

10. J. T. Hong, "Search for Slow-Moving Magnetic Monopoles with the MACRO Detector," contributed talk, 23rd International Cosmic Ray Conference, Calgary, July 1993. 


\title{
TASK D: NEUTRINO ASTROPARTICLE PHYSICS AND PROTON DECAY
}

\author{
Faculty: $\quad$ Professor L.R. Sulak (co-PI) \\ Associate Professor J. Stone \\ Research Associate Professor S.T. Dye (co-PI) \\ Research Associates: T. Miller or M. Masuzawa \\ Graduate Students: to be named
}

\section{Introduction}

Boston University continued to play a leadership role in the activities of the IMB collaboration. Implementation of the four-year plan outlined in our earlier proposal progressed very well. This plan called for active participation in Super-Kamiokande and DUMAND to eventually replace our efforts on IMB, which terminated data-taking in April 1991. The Super-Kamiokande initiative became reality with approval and funding of the proposed participation of the U.S. group. With Stone providing vigorous leadership as co-spokesman, Boston University played a pivotal role in this new endeavor. The joint Japanese-U.S. calibration of water Cerenkov detectors using charged particle beams at KEK advanced with minor schedule delays. The IMB-3 instrumentation, which will be used at KEK, was assembled for testing at Boston University. Our hardware contribution to DUMAND neared completion and preparations for data analysis were completed. In parallel with the efforts on the new initiatives, good progress was made analyzing IMB-3 data. Within the last year, four IMB papers were published in journals. In addition to an instrumentation paper describing the IMB-3 detector in detail, these papers reported analyses of the contained data, the upward-going muon data, and the downward-going muon data. Papers reporting a search for magnetic monopoles and astrophysical sources of low energy neutrinos were submitted for publication.

In the following sections we describe in more detail the progress on the new initiatives and on analyzing IMB data. We begin with an overview of Super-Kamiokande. A discussion of the accelerator-based calibration tests of a large water Čerenkov detector follows. Next, we outline the DUMAND fast time digitizer task. In conclusion, we present the latest physics results derived from analysis of IMB data. 


\section{Super-Kamiokande}

Super-Kamiokande is a 50,000 ton ring-imaging water Cerenkov detector to be constructed at a depth of 2700 meters water equivalent (mwe) in the Kamioka Mozumi mine in Japan. A schematic representation of the detector is shown in Figure D.1. It consists of a stainless steel tank in the shape of a right circular cylinder, $39 \mathrm{~m}$ diameter and $41 \mathrm{~m}$ height, filled with purified water. The detector is optically segmented into an inner volume $(34 \mathrm{~m}$ diameter, $36 \mathrm{~m}$ height) and an outer (anti-coincidence) region of $2.5 \mathrm{~m}$ thickness on top, bottom, and sides of the inner volume. The inner detector is viewed by 11,200 photomultiplier tubes (PMTs) of $50 \mathrm{~cm}$ diameter, uniformly distributed on the inner boundary giving a $40 \%$ photocathode coverage. This extraordinary photocathode coverage and time resolution (2.5 ns at 1 p.e.) allows the detector to attain an energy threshold of $5 \mathrm{MeV}$ and a vertex resolution of $10 \mathrm{~cm}$ for processes such as $\mathrm{p} \rightarrow \mathrm{e}^{+} \pi^{0}$. For through-going muons, the PMT configuration yields an angular resolution of $1^{\circ}$. The total mass of water inside the inner detector PMT surface is 32,000 tons. The fiducial mass for the proton decay search, defined to be $2 \mathrm{~m}$ inside the PMT plane, is 22,000, tons allowing for partial lifetime sensitivities of $\geq 10^{34}$ years for several modes.

The outer annulus of the detector is an anti-coincidence region used to tag entering muons and low energy components as well as to attenuate low energy gammas and neutrons which cause background in the sensitive volume. It also complements calorimetry in the inner detector by measuring the energy loss due to exiting particles. This outer detector region is viewed by 2200 PMTs of $20 \mathrm{~cm}$ diameter with wavelength-shifter plates in the style of IMB-3. The walls of the anti-coincidence region are made reflective to enhance light collection. The PMTs are mounted facing outwards on the same super-structure as the 50 cm PMTs of the inner volume. An optical barrier is mounted on the same structure to separate the inner and outer regions.

\section{Nucleon Decay in Super-Kamiokande}

Nucleon instability, expected at some level in many extensions of the standard model, still offers a unique probe in searches for the ultimate theory of interactions. In this respect, the observation of nucleon decay would have more significant and far-reaching consequences than those from experiments seeking to fill existing gaps in the standard model, for example by finding the top quark or the Higgs. The lifetime of the proton depends on the scale 
of grand unification as determined by the convergence of the three fundamental running coupling constants at a single point at very high energy. Recent measurements of these coupling constants at LEP have resulted in much better predictions of the proton lifetime than was previously possible, and these lifetimes are within the reach of a detector with the size and resolution of Super-Kamiokande.

Super-Kamiokande will be the largest nucleon decay detector ever constructed. In terms of its sensitive mass (22,000 metric tons) it is larger than the sum of the fiducial masses of all the other detectors ever built (NUSEX, Soudan I, IMB, Kamioka, Soudan II, Frejus, and $\mathrm{KGF}$ ). Super-Kamiokande will not only have seven times the fiducial volume of IMB, it will also have ten times the light collection capability. This will result in a substantially higher efficiency for low-light level decay modes. When coupled with lower background rates from atmospheric neutrinos (due to better energy and track resolution), this will lead to at least an order-of-magnitude increase in nucleon decay detection sensitivity for most decay modes. It is this ten-fold improvement that will allow Super-Kamiokande to test theories (such as Flipped $S U(5) \times U(1))$ that predict proton decay in the range $10^{33}-10^{35}$ years.

\section{Atmospheric Neutrinos in Super-Kamiokande}

The sheer size of Super-Kamiokande is very important for refined measurements of the atmospheric neutrino flux and composition. The IMB detector recorded about one contained atmospheric neutrino event per day of operation whereas Super-Kamiokande will record about seven. Systematic uncertainties will dominate the statistical uncertainty in SuperKamiokande but the improved statistics will allow the data to be cut into different classes for systematic studies.

However, it is not only size that separates Super-Kamiokande from the previous generation of underground detectors. The pattern recognition method of particle identification now suffers due to modest light collection (about 1-5 pe/MeV) and vertex resolution (about $1 \mathrm{~m}$ ); for example, a misfit event vertex could turn a muon track into an apparent electron track and vice versa. Thus, the efficiency for correct particle identification in IMB-3 was slightly better than $90 \%$ and in Kamiokande it is about $98 \%$. The enhanced light collection $(7 \mathrm{pe} / \mathrm{MeV}$ ) and greatly improved vertex resolution (about $0.1 \mathrm{~m}$ ) will mean the particle identification efficiency will be greater than $99 \%$. 
Other properties of Super-Kamiokande are important for the study of atmospheric neutrinos. The muon decay detection efficiency for $\mu^{+}$in IMB-3 was about $80 \%$, and in Kamiokande it is $87 \%$. However, the faster electronics, better light collection, and lower energy threshold in Super-Kamiokande will allow a $\mu^{+}$decay to be identified $95 \%$ of the time. This will mean that this simple method of determining the particle identification will be about as efficient as the current pattern recognition methods.

The underground measurements of the atmospheric neutrino spectrum and composition by Kamiokande and IMB-3 are in good agreement. The disagreement between these measurements and the theoretical predictions is, however, statistically quite significant. The cause of the disagreement must be either an unidentified systematic effect in the flux calculation, neutrino model, or detector simulation, or some new physics.

Investigation of systematic effects (the neutrino model or detector simulation) requires controlled experiments. Such experiments include a charged particle beam test, now underway, and a future neutrino beam test. Both of these tests are going to use a large water Cerenkov detector at KEK. Isolation of potential systematic problems with the neutrino flux calculations will require, among other things, much more atmospheric neutrino data to search for energy, zenith angle, or other effects that might indicate the nature of the problem.

The unambiguous confirmation that the atmospheric neutrino problem is the result of some exciting new physics will require a vast increase in the size of the neutrino event sarnple. The most compelling explanation involving new physics is that neutrino oscillations are causing the $\nu_{\mu}$ 's to disappear before they reach the underground detectors. Several complementary approaches are likely required to completely probe this possibility. One approach would use the greater statistics in the neutrino sample, along with the greatly improved resolutions and particle identification efficiencies of Super-Kamiokande, to allow a detailed systematic search for possible energy or path-length deviations that would indicate neutrino oscillations.

Another approach to this problem will be accelerator-based long-baseline neutrino oscillation experiments. Because of the range of neutrino masses implied by the atmospheric anomaly, such an experiment will require that the neutrino beam energy and path length satisfy $L / E \geq 100 \mathrm{~km} / \mathrm{GeV}$. These possibilities are being investigated in proposals at FNAL, 
BNL, and CERN for long-baseline neutrino experiments. An $\sim 1 \mathrm{GeV}$ neutrino beam from KEK pointed at Super-Kamiokande would fulfill this requirement with the added advantage of using an existing, well understood, very large detector with excellent resolution.

Clearly Super-Kamiokande provides a unique opportunity to study atmospheric neutrinos in great detail. Hints of the atmospheric neutrino problem were first uncovered in data from the IMB detector. It is very likely that the solution will come with data from Super-Kamiokande.

\section{Boston University's Hardware Contribution}

Boston and Stony Brook are the lead institutions responsible for developing the low energy triggering system for Super-Kamiokande. A $5 \mathrm{MeV}$ trigger is needed to increase the detector's sensitivity to nucleon decay. When, for example, a proton decays inside the oxygen nucleus, the isotope ${ }^{15} \mathrm{~N}$ is formed. There is a $50 \%$ probability that ${ }^{15} \mathrm{~N}$ is excited to the $P_{3 / 2}$ state which decays immediately by emission of a $6.3 \mathrm{MeV} \gamma$. Detection of this prompt low energy $\gamma$ is crucial to reducing background to $p \rightarrow \nu K^{+}$where the $K^{+}$decays to $\mu^{+} \nu$. Since the $K^{+}$is a heavily ionizing particle it decays at rest with high probability. Thus the $\mu^{+}$is monoenergetic and follows the $\gamma$ on average by the $K^{+}$lifetime of $12 \mathrm{~ns}$. The coincidence of a $236 \mathrm{MeV} / \mathrm{c}_{\mu^{+}}$preceded by a $6.3 \mathrm{MeV} \gamma$ is an unambiguous signal of $p \rightarrow \nu K^{+}$. Ability to search efficiently for this coincidence removes the limitation from the atmospheric neutrino background for this important SUSY decay mode.

An energy threshold of $5 \mathrm{MeV}$ is the goal of the trigger for the inner detector of SuperKamiokande. The trigger design is constrained by the input signals from the PMTs through the front-end electronics. Both the PMTs and the front-end electronics are briefly described below.

The inner detector of Super-Kamiokande will employ the new $50 \mathrm{~cm}$ Hamamatsu R1449 photomultiplier tube. This tube, an improved version of Hamamatsu's original $50 \mathrm{~cm}$ PMT developed for Kamiokande, resulted from a four-year development program between KEK, Tokyo Institute of Technology, and Hamamatsu.

The most important tube characteristic for event reconstruction in an inaging Cerenkov detector is the time jitter (transit time spread). This is significantly improved in the R1449. 
The measured jitter (at $1 \mathrm{pe}$ ) is $\sim 2.5 \mathrm{~ns}(1 \sigma)$. This is to be compared with a $4 \mathrm{~ns}$ jitter for the original $(50 \mathrm{~cm})$ Kamioka PMTs and the $\mathrm{T}$ ns of the Hamamatsu 8 -inch tubes used by IMB.

Energy resolution is a second important factor, enhancing sensitivity to low energy phenomena and the discrimination against backgrounds from the radioactivity of the detector components and from the outside environment. The pulse height response of the new tube shows a significant improvement in resolution over the old Kamioka tube, with a single-pe peak which is well separated from the dark noise distribution. The combination of improved precision in event reconstruction and improved energy resolution are essential in realizing the $5 \mathrm{MeV}$ energy design threshold for Super-Kamiokande.

The average dark noise for the initial production run of new tubes was $20 \mathrm{kHz}$, higher than the target rate of $3 \mathrm{kHz}$. A factor of 4 reduction will be achieved in the detector where the water temperature is maintained at $11^{\circ} \mathrm{C}$. Previous experience with the $50 \mathrm{~cm}$ tubes shows that the average dark count rate can be expected to decrease as the production run proceeds. The accidental trigger rate for low energy phenomena is a strong function of individual PMT dark noise and this situation will be monitored closely.

The front-end electronics accept PMT pulses, convert them to digital form, place the data onto the digital pathways of the data acquisition system, and provide PMT hit information (HITSUM/PMTSUM) to the detector triggering system. Figure D.2 shows the basic unit of the front-end electronics: the Analog Timing Module or ATM. Each ATM handles 12 PMT channels, a group of which is called a "supertube". PMTSUM is an analog sum of up to 12 PMT pulses. Pulses of $10 \mu \mathrm{A}$ (peak) are produced for $1.6 \mathrm{pC}$ PMT pulses which correspond to 1 pe. The sum of PMTSUM pulses is a current proportional to the instantaneous Cerenkov energy deposition in the detector. HITSUM is a current source which is modulated by the number of discriminators in the on-state. In the off-state, there is no current, and for each discriminator which is on, the current is increased by $100 \mu \mathrm{A}$.

Although the Cerenkov light patterns from different types of events are quite distinct, pattern recognition at the trigger level is complicated. The simplest trigger could, as was done in IMB, merely require a certain number of PMTs above thre shold within a coincidence time window. If it is assumed that each PMT corresponds to 1 pe, then the total number of 
50.000 ion Water Cherenkov Detector

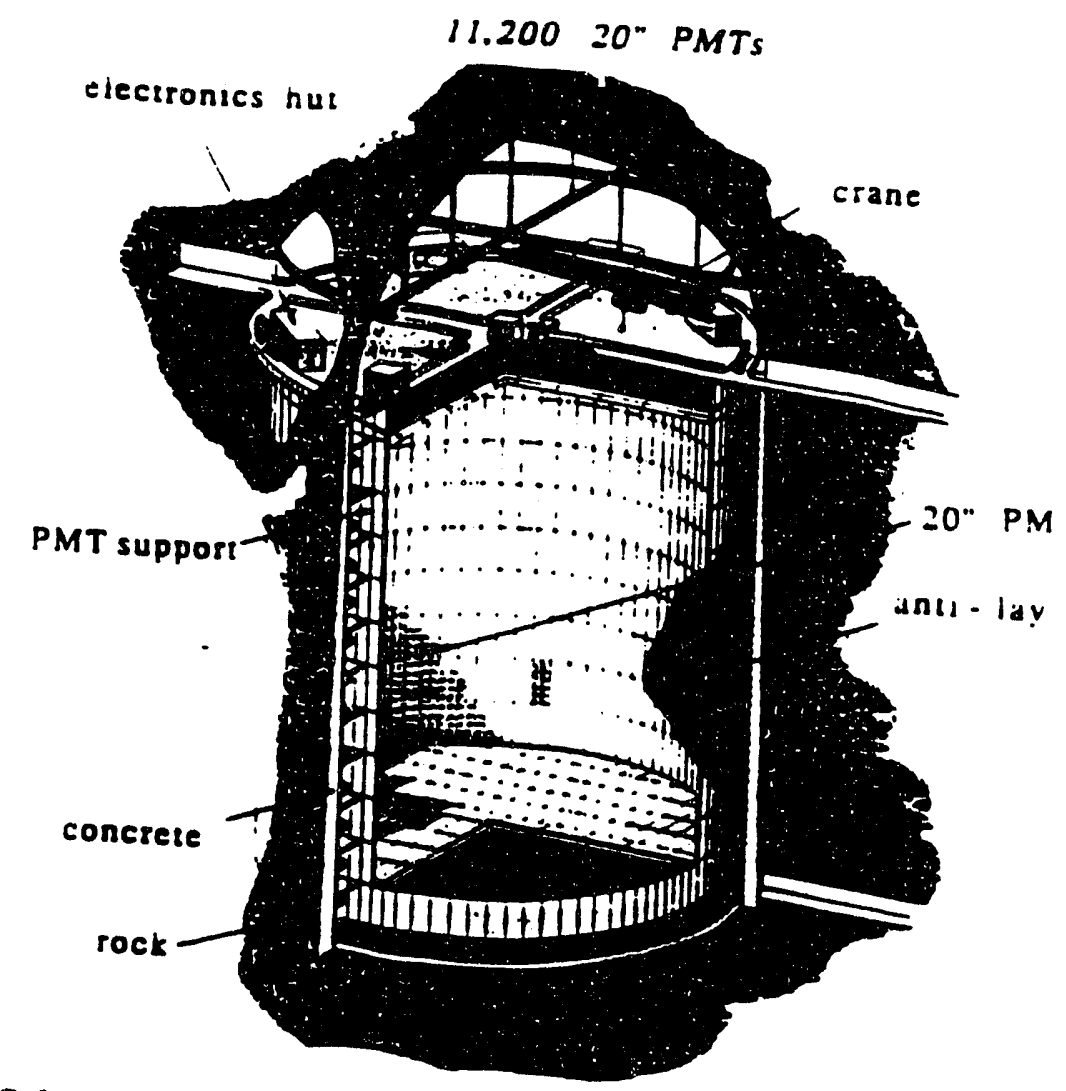

Figure D.1 Schematic representation of the Super-Kamiokande detector.

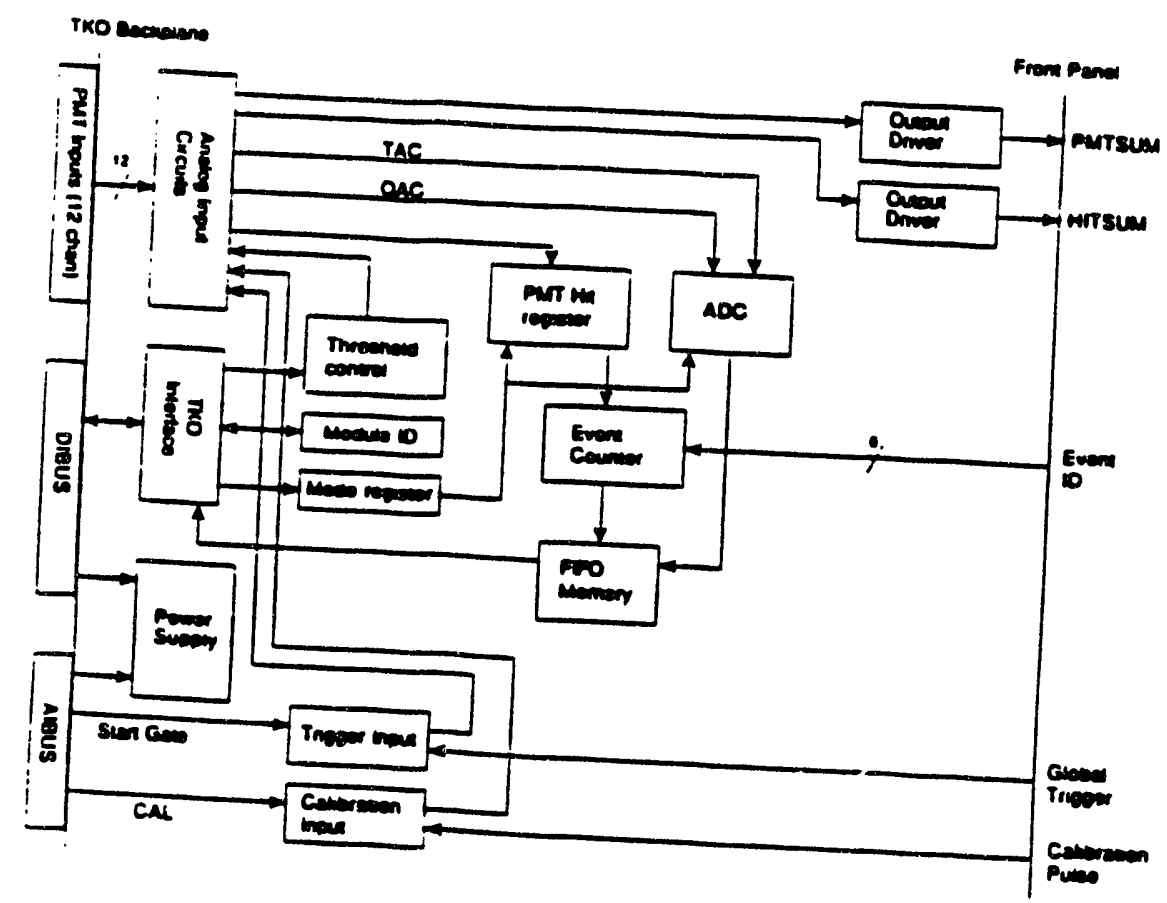

Figure D.2 Block diagram of the Analog Timing Module (ATM) used in the front-end elecTask D: Neztrino Aetrophyolcs and Prolon Decey 
PMTs can be related to energy. In Super-Kamiokande $1 \mathrm{MeV}$ of energy deposition produces roughly 7 pe. In this case a $5 \mathrm{MeV}$ trigger threshold corresponds to about 35 PMTs in coincidence.

Because of the large number of PMTs in Super-Kamiokande and the large size of the detector, which dictates the duration of the coincidence time window, the accidental trigger rate due to PMT dark noise needs to be addressed. The photon flight time across the greatest distance between PMTs in the inner detector is about $210 \mathrm{~ns}$. This sets the duration of the coincidence time window. If the average PMT dark noise rate is $5 \mathrm{kHz}$, a $35 \mathrm{PMT}$ coincidence in $210 \mathrm{~ns}$ produces an accidental trigger rate of almost $10 \mathrm{~Hz}$. If an average PMT dark noise rate of $3 \mathrm{kHz}$ is realized, then the accidental trigger rate falls by some four orders of magnitude. Since there is some uncertainty in the actual PMT dark noise rate it seems prudent to design the trigger system to function properly with an average PMT dark noise rate of $10 \mathrm{kHz}$. However, the accidental trigger rate is about $1 \mathrm{MHz}$ when the average PMT dark noise rate is $10 \mathrm{kHz}$. Clearly a simple coincidence of 35 PMTs in $210 \mathrm{~ns}$ is not a feasible trigger algorithm for Super-Kamiokande.

The data acquisition system consists of a control station and four substations. Each substation services one quadrant of the detector (2800 PMTs). The trigger system is also made of four substations and a control station which issues the global trigger signal. It is perhaps more natural to form trigger decisions based on PMT activity in quadrants or smaller segments of the detector. The number of PMTs and the coincidence time window are reduced, which alleviates the accidental trigger rate problem. Figure D. 3 plots the accidental trigger rate for $1 / 4,1 / 8$, and $1 / 16$ of the detector as a function of the number of PMTs in coincidence. It is possible to trigger directly on coincidences within the individual segments or to form coincidences between pairs of segments. Figure D.4 plots the accidental trigger rate for coincidences between segment pairs of $1 / 4,1 / 8$, and $1 / 16$ of the detector as a function of the accidental rate in the individual segments. By employing multiple trigger algorithms it seems possible to achieve a threshold of $5 \mathrm{MeV}$. The efficiencies of the algorithms need to be estimated through Monte Carlo simulation of low energy events near threshold. However, it is clear that the trigger system must be versatile if the goal of $5 \mathrm{MeV}$ is to be achieved.

The trigger electronics must efficiently detect energy deposition of $5 \mathrm{MeV}$ or more in the detector and issue a signal to the ATMs within $1 \mu \mathrm{s}$. Other design criteria include Page 3-8 


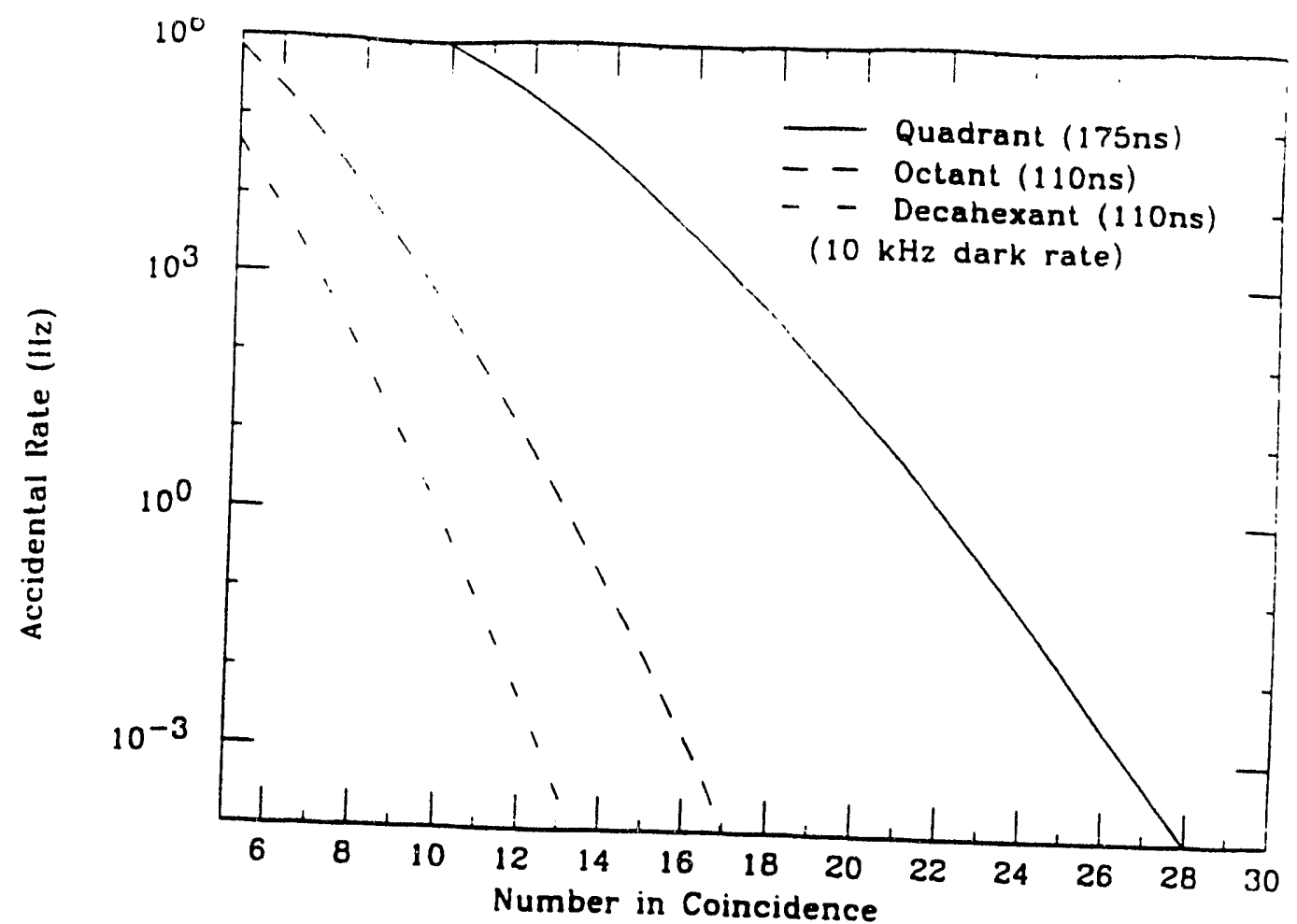

Figure D.3 Accidental trigger rate of different size segments of the Super-Kamiokande detector for an average PMT dark noise rate of $10 \mathrm{kHz}$.

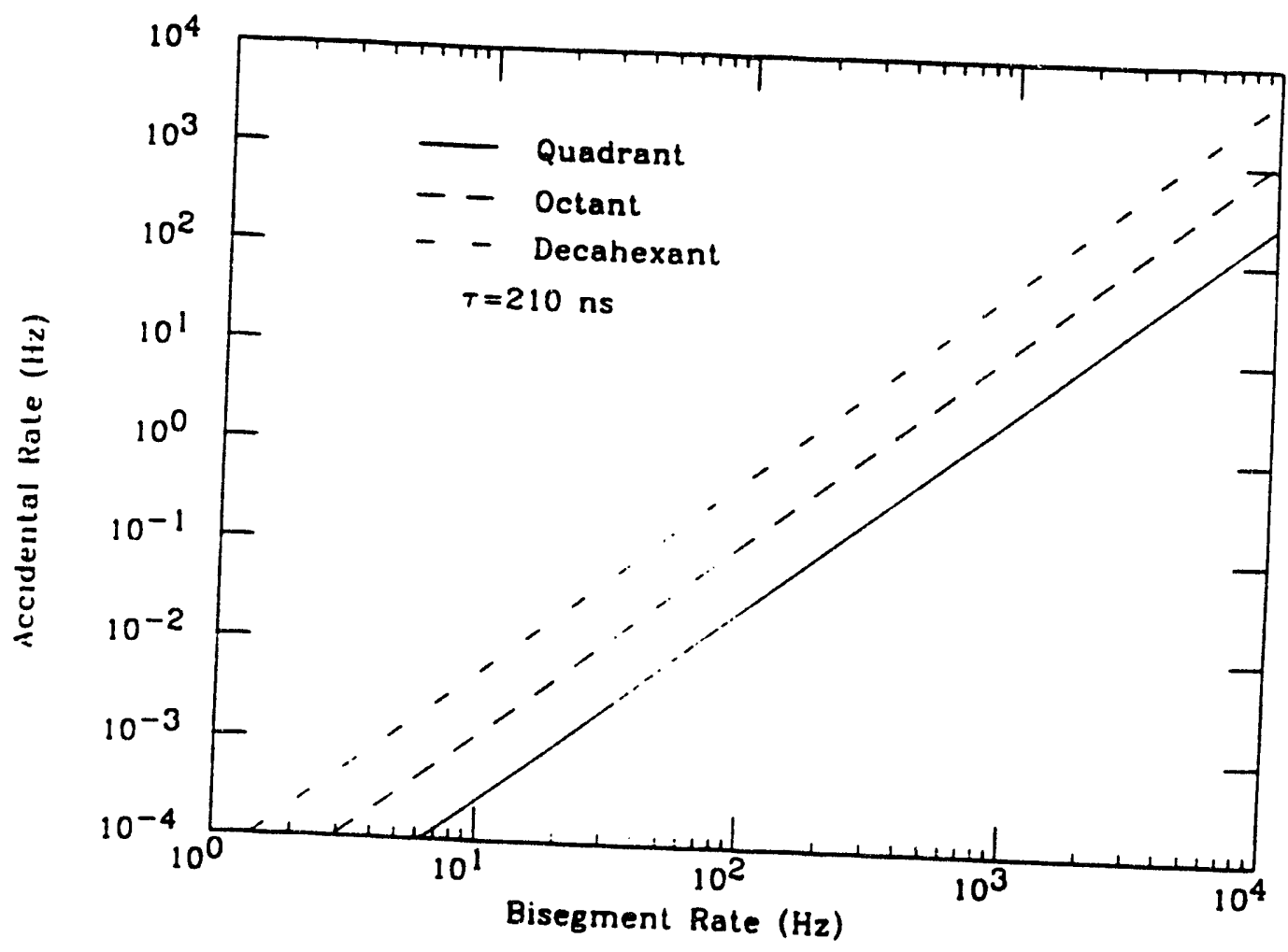

Figure D.4 Accidental trigger rate for pairs of segments in coincidence in the Super-Ka-
miokande detector.

Task D: Nextrino A atrophyoics and Proton Decay 
reliability, serviceability, and versatility. Trigger systems which satisfy these requirements are being investigated by the Boston University electronics shop. One possible design is shown in Figure D.5. It employs current-voltage converters, discriminators, and flash ADCs for the substations, and programmable trigger logic for the control station. The HITSUM current pulse from each supertube is converted to a voltage pulse which is discriminated. The discriminator output current is proportional to the multiplicity of input signals above the preset threshold. Current outputs from all discriminators are combined and digitized by the flash ADC which sends the digital information to the programmable trigger logic in the control station. If any trigger conditions are satisfied, a global trigger signal is issued to the data acquisition substations.

\section{Beam Tests at KEK}

The U.S. IMB group has joined forces with the Japanese Kamioka group to perform a series of accelerator beam tests of water Čerenkov detectors. The objectives are several fold. The initial beam test at KEK will measure the identification efficiencies for muons and electrons in a scaled down (10 m diameter, $10 \mathrm{~m}$ height) water Čerenkov detector using both

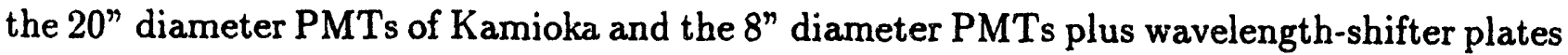
of IMB-3. These data will serve to calibrate the Kamioka and IMB-3 detectors, respectively. The goal of both the Japanese and U.S. groups is to study any systematic effects related to muon and electron identification in order to understand better the apparent anomalous measurement of the $\nu_{\mu}$ to $\nu_{e}$ ratio in the atmospheric neutrino flux. The schedule of tasks for these tests, which will be in the spring of 1994, is presented in Figure D.6. In addition, a proposal is being submitted to KEK for a neutrino beam exposure using the water Čerenkov detector. This test would allow the direct measurement of neutrino - oxygen cross sections and topologies which could be used for background corrections of the proton decay searches of Kamioka and IMB experiments as well as for the atmospheric neutrino composition question.

For the 1994 calibration beam test, the U.S. group has the responsibility to provide all components of the IMB-3 detector (PMTs, wavelength-shifters, cables, HV, readout electronics, data acquisition, etc.) required to perform the tests. In addition, the U.S. group will provide the reverse osmosis water purification system and laser calibration system to be used by both groups. The Japanese will provide the water tank, utilities, and the required 


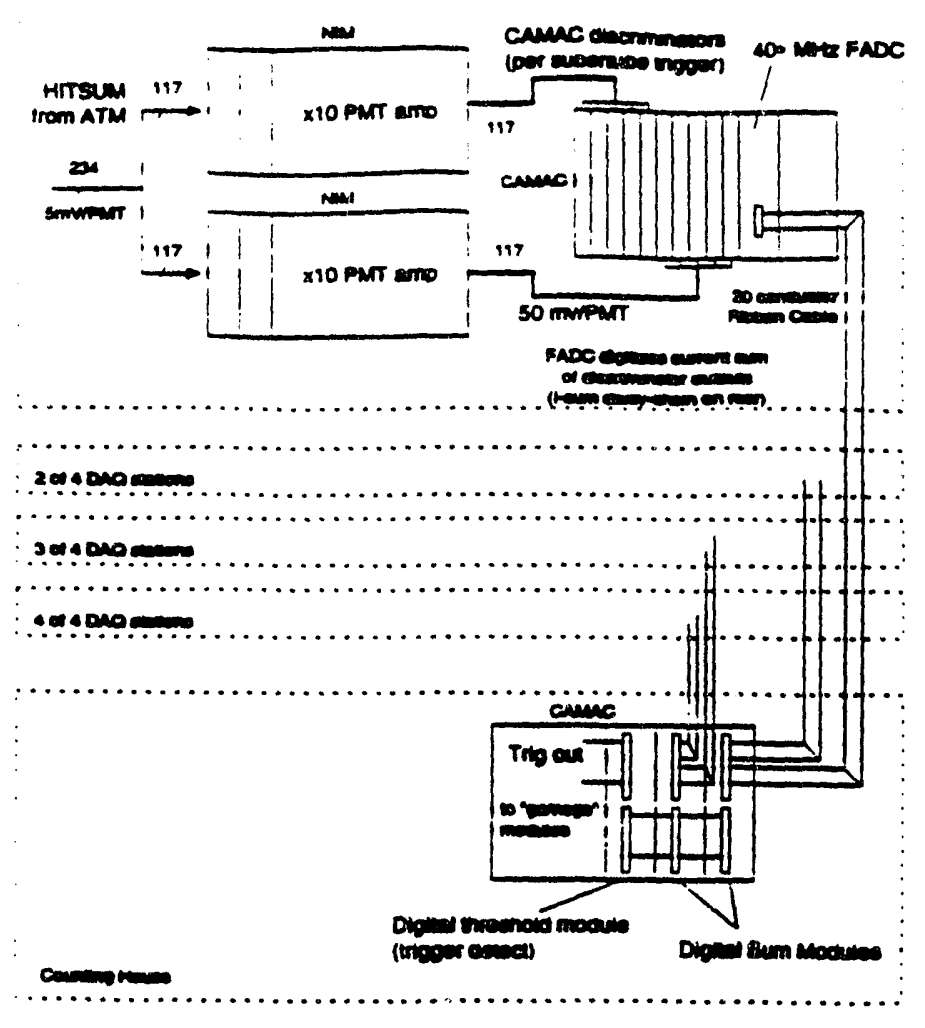

Figure D.5 Block diagram of a possible trigger system for Super-Kamiokande.

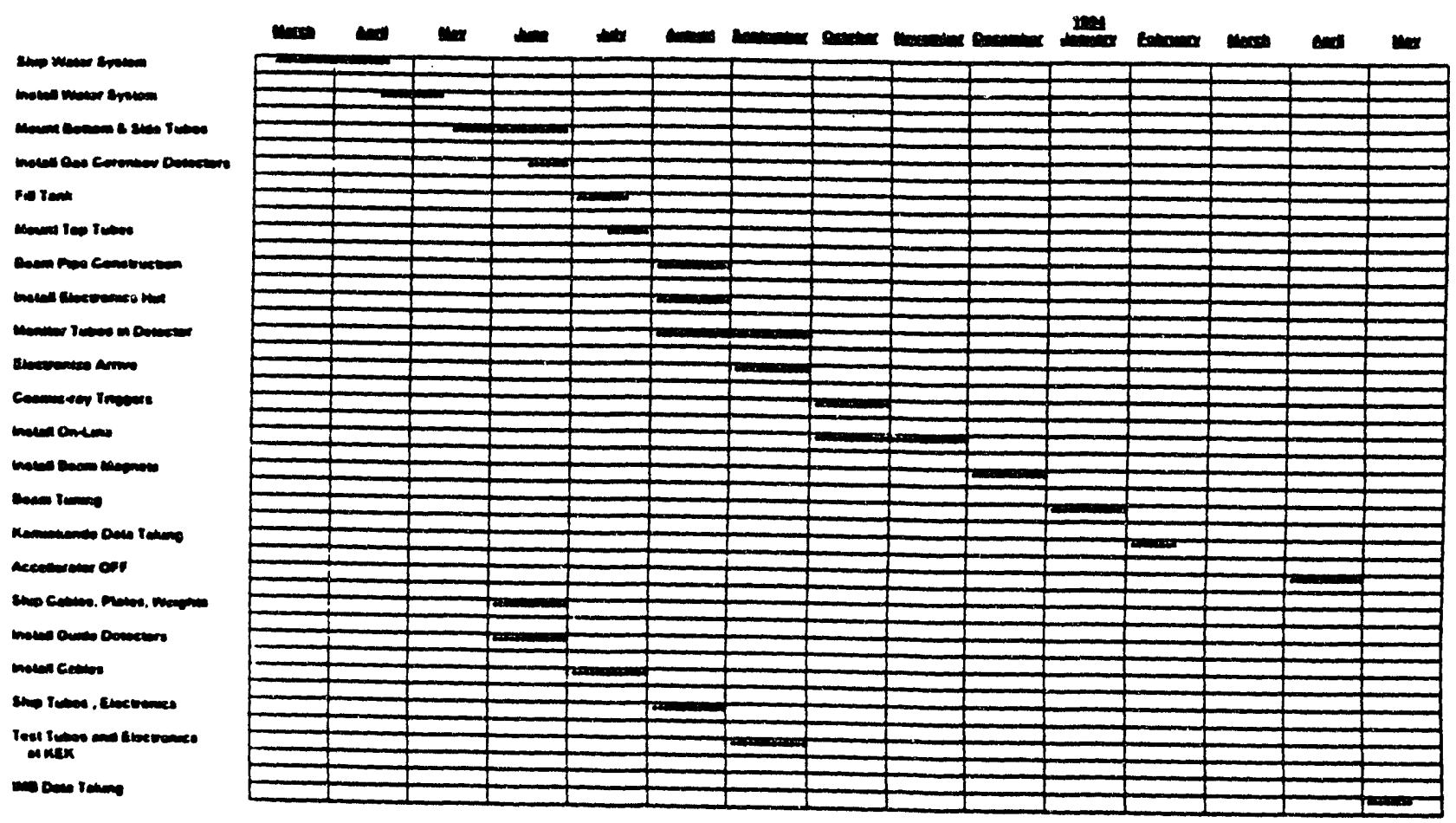

Figure D.6 Schedule of activities and tasks for the beam test at KEK. 
components of the Kamioka detector. The Boston University group has assumed responsibility for the PMTs and related cables. bases, HV, and digitizing electronics (ADCs and TDCs known as IMB custom electronics).

\section{Physics Background}

The IMB search for nucleon decay has yielded a large sample of atmospheric neutrino interactions contained in the detector volume (500 events in IMB 1-2 and 935 events in IMB-3). These interactions are the major background in a search for nucleon decay, yet they are also a source of physics. For example, the atmospheric neutrinos which impinge on the IMB detector isotropically, allow for a unique study of neutrino oscillation. Produced in the upper atmosphere by primary cosmic ray interactions, they travel distances ranging from a few $\mathrm{km}$ (directly above) to $12000 \mathrm{~km}$ (directly below). Such large oscillation distances are not available with other neutrino beams in this energy range and for these neutrino flavors. The low mean neutrino energy coupled with the large distances allow a study of oscillations with $\delta \mathrm{m}^{2}$ as low as a few $\times 10^{-5} \mathrm{eV}^{2}$. Although there are some drawbacks, IMB's large sample of contained neutrino interactions constitutes a valuable source of physics. The most accurately predicted feature of the atmospheric neutrinos $( \pm 5 \%)$ is the ratio of the muon neutrino to electron neutrino flux. If oscillations of any kind occur, this ratio could change.

Using a subsample of events with one visible ring, IMB has developed a method of identifying "showering" (due to $e^{ \pm}, \gamma$ ) from "non-showering" (due to $\mu^{ \pm}, \pi^{ \pm}$) rings. This method uses the pattern of illuminated phototubes caused by the difference in the mechanisms of energy deposition by these two classes of events. In the visible energy range $100 \mathrm{MeV}<E_{c}<$ $1500 \mathrm{MeV}$, IMB observes about a $3 \sigma$ deficit in the non-showering fraction. A similar, more significant deficit is observed in the Kamioka data. The Kamiokande collaboration suggests that the deficit could be a manifestation of muon neutrino oscillation, with most probable $\delta m^{2} \sim 10^{-2}$ and mixing as high as $\sin ^{2}(2 \theta) \sim 0.69$.

Many of the same problems of particle identification are involved in understanding the atmospheric neutrino background to nucleon decay. There are no good low energy neutrino data available on water or a similar target. Deficiencies in the neutrino interaction models, including difficulties modeling nuclear effects limit our precision in predicting the background to various modes of nucleon decay. 


\section{The KEK Experiment}

An experiment, jointly proposed by the Japanese and U.S. groups, will study systematic effects of particle identification in water Cerenkov detectors. The experimental technique will consist of directing beams of charged particles: $e, \mu, \pi$ of well defined momenta in the range of $100-2000 \mathrm{MeV} / \mathrm{c}$, and low energy neutrinos $\left(E_{\nu} \leq 2 \mathrm{GeV}\right)$ into a 1000 ton water Cerenkov detector. The detector will be instrumented with $20^{\text {" }}$ Hamamatsu phototubes with photocathode coverage equal to that of the Kamiokande-II detector and later with 8 " phototubes and wavelength-shifter plates as in the IMB-3 detector. The readout and control electronics will be the same as used in (:ach of these experiments. The charged particle beams will allow us to collect data in the setector from identified particles of well known momenta and geometry. The particles will be injected into the detector by means of a beam pipe penetrating the detector wall at 6 different locations and 3 different depths. The analysis of the data collected in this way will allow us: i) to verify track reconstruction procedure; ii) to verify track identification meihods; iii) to provide a precise energy calibration; iv) to veriry the light tracking Monte Carlo simulation; and v) to verify the muon decay detection efficiency. All of these factors will allow us to re-analyze the IMB-3 data with improved knowledge of systematic uncertainties.

The neutrino beam exposure will allow us to collect a sample of neutrino interactions in the detector volume which include the neutrino-oxygen cross sections and topologies. The beam will be well understood and have a small $\nu_{e}$ component. Analysis of this data will allow us: i) to study the neutrino interaction in water and the outgoing lepton momentum distribution; and ii) to study final states of the neutrino interactions to provide better predictions of nucleon decay background from atmospheric neutrino interactions. With these data, we anticipate that background corrections cor"d be made to IMB-3 results yielding improved limits for several nucleon decay modes. A neutrino beam at KEK offers an exciting possibility to perform a long baseline neutr ino oscillation experiment in conjunction with Super-Kamiokande.

\section{Boston University's Hardwre Contribution}

The Boston University group has taken on complete responsibility for the front-end electronics, high voltage system, HV/PMT signal cables, preparing and testing all PMTs, 
and for mounting of the top and bottom PMTs. These responsibilities were natural for our group due to our past experience on the IMB and MACRO projects.

The front-end electronics and HV system are clean, tested, working, and ready for service. A selection of the highest quality front-end electronics is to be used at KEK. The HV/PMT signal cables are clean, tested, repaired, and at KEK. Each cable was completely tested for water tightness since small nicks or pinholes, which allow water to fill the PMT housing, could damage the PMT. The cable connectors and PMT water-tight housing nylaflow fittings were replaced, since the originals were sacrificed during the emergency removal of the PMTs from the IMI detector. The PMT bases have been tested and repaired as necessary.

Tive entire system to be shipped to KEK is being integrated and tested at Boston University. Office space hos been converted to a dark room which now contains all 400 of the selected PMTs to be used at KEK. These PMTs have all been tested and are working. The IMB laser system has been installed and, in addition to a LED system, is capable of triggering the system for testing.

The mechanical mounting of the PMTs in the water tank at KEK has been studied. We plan to use the same technique of neut-al buoyancy and suspension of the PMTs which was in use for the IMB detector. Yowever, this system must be designed and integrated with the Japanese scheme for the tank and mounuing of their PMTs since both sets of PMTs will be in the KEK tank at the same time. A method has been found to accommodate both the IMB and Kamioka PMTs with one set covered with a light barrier while the other set is taking beam. Wie believe that we have a method which will work, however, it will require that we build a superstructure above the tank at KEK similar to the catwalk - rail system which we used for the IMB detector. In this way, we will be able to re-use many of the components from the IMB salt mine facility.

One of the more challenging aspects of placing IMB PMTs in the KEK tank which is cylindrical as opposed to the rectangular IMB detector is the suspension of the top and bottom planes. We plan to re-use the PVC beam structure of IMB by having parallel beams of different lengths. Light barriers made of stiff, black plastic sheets will be attached to each beam. To implement this scheme, certain modifications to the beams will be made in the 
Boston machine shop. Although unknown at this time, it may be necessary to have a diver in the KEK tank to assist the installation of the top and bottom planes of PMTs.

\section{DUMAND}

DUMAND II is an array of 216 optical modules deployed in nine vertical strings that will be placed in an octagonal configuration, of $100 \mathrm{~m}$ diameter with one string in the center, at a depth of $4760 \mathrm{~m}$ at the bottom of the ocean $25 \mathrm{~km}$ from the island of Hawaii (see Figure D.7). The primary purpose of the experiment is to search for astronomical sources of very high energy and ultra high energy neutrinos. Data-taking is expected to begin this fall when the first three strings with 72 optical modules are scheduled for deployment.

The unexpected observation by GRO of intense gamma-rays in the $100 \mathrm{MeV}$ to $10 \mathrm{GeV}$ region emitted by over a dozen active galaxies has demonstrated that these sources emit more power at high energies than in any of the lower energy spectral bands. One of the GRO sources, Mrk421, has been reported at TeV energies. These sources are likely to be emitting very high energy neutrinos of comparable luminosity. Models of the central engines of active galaxies strongly suggest that these are also sources of VHE and UHE neutrinos that will be detectable by the nine string DUMAND II array.

\section{Summary of the Status of DUMAND II Construction}

The design of DUMAND II is complete and components for the first three strings are currently being assembled and tested. Optical modules (OM's) from Japan and Europe are being shipped to Hawaii for final testing and incorporation in the strings. Final testing is being accomplished in Hawaii in a $15 \mathrm{~m}$ long water tank using $10 \mathrm{~m}$ trigger scintillation counters given to us by the MACRO group at CalTech. String hardware components are all on hand as of March 1993, and final assembly is about to begin. The instrumentation on one string consists of $24 \mathrm{OMs}$, plus 1 or 2 laser calibration modules (on alternating strings), 5 hydrophones, and some environmental monitoring transducers. There is a flotation package at the top, with beacons and recovery hardware. The string base has anchoring, an acoustic release for emergency retrieval (which can also be accomplished with a submersible), and umbilical cable and connectors. A $100 \mathrm{~m}$ tare height is left without instruments above the anchor. The OMs are spaced $10 \mathrm{~m}$ apart, and the floats occupy another $100 \mathrm{~m}$ above, for a total string length of $430 \mathrm{~m}$. 
Signals from the modules for each string are transmitted via multi-mode fiber optics to a string electronics package, located in the geometric center of each string. A $2 i$-channel pipelined VLSI digitizer chip with 1 ns resolution has been designed and tested at Boston University. It records the arrival time and duration of each $O M$ pulse, with appropriate buffering, and passes 2 words every 80 ns to a 40 bit parallel-to-serial converter for transmission to shore at $500 \mathrm{Mbd}$. A specially designed cable carrying 12 single mode fibers and capable of delivering $10 \mathrm{~kW}$ of power has been constructed. On shore the data is to be time corrected to Universal Time, and passed to digital trigges processing units. There are several triggering algorithms, but the basic first level simply looks for nearest neighbor coincidences upon strings. The second level seeks various combinations of such coincident hits. The shore station is presently under construction, but a pipe for bringing the cable through the surf zone has already been installed.

An acoustic positioning system, which will allow for continuous determination of the position of the elements of the array to within $10 \mathrm{~cm}$ is undergoing final testing. The hydrophones will also be employed to search for acoustic pulses associated with UHE interactions.

Deployment, slated for October 1993, will begin with placement of the central string of instruments, an environmental package with TV and lights at its base, and a junction box at the bottom. The junction box carries the shore cable termination, sea water return electrode, and 12 electro-optic connection ports (of which two are spares). The main cable will then be laid in to shore. The instruments will be monitored for functionality during the operation. The process is designed to be reversible if problems are encountered. Following this, two more strings will be put into position on the ocean floor, $50 \mathrm{~m}$ away from the junction box. In the final step, a submarine, or alternatively a deep ocean remotely-controlled vehicle, will be employed to pull umbilical cables from the string bases to the junction box and make the electro-optic connections. There are also 4 outrigger transponding packages to be connected to the junction box from their $300 \mathrm{~m}$ distant locations. The connection operation was successfully practiced at the DUMAND site in October 1992.

Much effort has been devoted to reliability considerations in design and testing, with particular attention to corrosion resistance. For the latter purpose, most components are either non-metallic or made of titanium. Pressure and temperature cycling of all components is a requirement. 
Capabilities of the Three String Array

The full nine strings of DUMAND II are needed to obtain the high effective area $(20.000$ $\mathrm{m}^{2}$ at $3 \mathrm{TeV}$ ) and angular resolution $\left(1^{\circ}\right)$ needed to begin serious very high energy neutrino astronomy. Yet, the three strings to be deployed this fall will have some limited capabilities. Monte Carlo calculations indicate that through-going muons can be reconstructed with reasonable precision and with good background rejection for those events that produce multiple coincidences on adjacent modules in all three strings. For example, we can demand that two strings have at least a three-fold coincidence, while the third string as at least a two-fold. Or, we can demand a five-fold and two two-folds. In this case, the background of fake events will be sufficiently low to allow for the detection of a signal of ten events per year from a point source.

The effective area and angular resolution for events reconstructed according to these criteria are strong functions of energy. While the nine-string octagon has good capabilities down to $100 \mathrm{GeV}$, the three string triad requires muons of at least $3 \mathrm{TeV}$ for significant detection efficiency and pointing accuracy. The effective area at $10 \mathrm{TeV}$ is about $3100 \mathrm{~m}^{2}$ and scales roughly with $\log$ (energy). Note that our definition of effective area includes all identified efficiencies through reconstruction of events. The median pointing accuracy is $5.5^{\circ}$ at $1 \mathrm{TeV}$ and $3.6^{\circ}$ at $10 \mathrm{TeV}$. The atmospheric neutrinos, which are the primary background for extraterrestrial sources, have a much steeper spectrum than expected from these sources. The Triad will also have substantial capability for discovery of UHE cascades at distances of several hundred meters, and could provide the first evidence for diffuse AGN fluxes.

Muon detection also permits studies of downward-going cosmic ray muons for muon astronomy and cosmic ray composition studies. Upward-going muons will be examined for evidence of neutrinos from WIMP annihilations. Other than searching for muons and cascades (light from particles traveling at $c$ and point sources of light), we will have triggers to search for slow-moving sources $\left(\beta>10^{-3}\right)$, and for time clusters of high amplitude hits in OMs (possible supernova detection).

\section{Boston University's Hardware Contribution}

A monolithic multi-hit digital time-to-digital converter (TDC) has been developed for the DUMAND II experiment at Boston University. It has a pipelined architecture, 27 channels, 
and Ins least count. Buffers handle up to 48 input pulses. A maximum data rate of 100 $\mathrm{kHz}$ per channel is accommodated. Readout is continuous, deadtime-less, and time-ordered. The mean time between failures is greater than ten years.

Reliability and speed requirements made a monolithic digitizer an obvious choice. The requirement of continuous operation eliminated conventional ramp-type TDCs. We studied several all-digital solutions involving various combinations of custom and off-the-shelf integrated circuits (ICs), and finally decided to attempt an implementation based on a single large IC, containing the entire digitizer, multiplexer, and memory. We estimated that the IC would require about 60,000 two-input equivalent gates, consistent with available applicationspecific IC (ASIC) technology.

We studied several digital IC fabrication techniques: full-custom, standard cell, and gate arrays. In a full-custom design, an experienced IC design team is required, as all details of the layout must be specified to the manufacturer. This option is quite expensive. A standard-cell design is built from complex pre-tested circuit elements, too expensive and not appropriate to our design. A gate array design is built of pre-placed transistors configured for easy use as logic gates. The designer is provided with a library of "macros," pre-configured simple logic elements such as ANDs, ORs and flip-flops. The gate array is relatively inexpensive, as only the metal interconnection layers are custom. We found this to be a good fit to our design. We considered CMOS, ECL (emitter coupled-logic) and GaAs IC technologies. CMOS is too slow unless one resorts to complex multi-phase clocking schemes, although it has been used in a successful commercial digitizer, the LeCroy MTD132. ECL and GaAs both met our speed requirements. The substantially higher power consumption of ECL led us to opt for a GaAs device. One manufacturer, Vitesse Semiconductor, provides DCFL (direct-coupled FET logic) gate arrays with high speed, modest power requirements, and the required size. After much research, we chose the Vitesse VGFX200K DCFL gate array.

The digitizer processes input signals in parallel and packages the information in compressed form for transmission to shore. One nanosecond least count resolution suggests a 1 GHz clock, but this is impractical with today's IC technology for several reasons. Currently available large IC packages attenuate high-frequency signals excessively. Even using GaAs, the propagation delay between logic levels is excessive for a 1 ns clock period in a complex 
design. In addition, distributing a $1 \mathrm{GHz}$ clock over a large IC introduces significant uncertainty in clock timing across the device. Thus we selected a two-phase approach. The master clock runs at $500 \mathrm{MHz}$, and both edges are used. This significantly increases the complexity of the total circuit, as the rising and falling edges are handled in parallel. Additional circuitry ensures the preservation of event chronology, so that the data arrive at the shore in time order. The essential blocks of the digitizer chip, which are shown in Figure D., are:

Edge Detector This block assigns the asynchronous signals coming from the optical modules to discrete time bins. The edge detector also provides zero- suppression by triggering the circuit only when a transition occurs on an input. Both rising and falling edges are recognized, using separate circuits.

Time Stamp (not shown) A ten bit time stamp is recorded for each clock nanosecond containing valid data. The 10 bit time will roll over every 1,024 ns, and a special word is inserted in the data stream when this occurs. This allows the trigger processor on shore to accurately reconstruct event timing.

Hydrophone Inputs The roll-over words mentioned above are used to transmit auxiliary data to the shore station, including hydrophone data for sonar location of optical module positions.

First FIFO This block provides buffering for bursts of input data. The priority encoder services one channel per 2 ns clock cycle, which implies a need for queuing if transitions arrive in bursts. For the expected time distribution of input data, ten words in front of the priority encoder are sufficient. Two separate FIFOs store data recorded on rising and falling clock edges.

Priority Encoder This block identifies those channels where a transition took place. If there is more than one transition within the same nanosecond, the priority encoder emits a separate word for each transition, in order from low to high channel numbers. Since this is a true priority encoder, no scanning of empty bit locations is required, and each transition requires only 2 ns to encode.

Second FIFO The priority encoder generates data in bursts, but the bandwidth of the communication channel to shore need only suffice for the average speed of data generation if there is sufficient buffering in front of it. Simulation with Monte Carlo event data showed that a 100 word buffer was sufficient. 
Output Interface The digitized data are transmitted by optical fiber to shore at $500 \mathrm{Mbd}$, using a commercial GaAs transmitter-receiver chipset called the HotRod. The output interface delivers data to the Hot-Rod in the format it expects (one 40 bit word every $80 \mathrm{~ns}$ ). Each PMT input transition (rising or falling edge) generates a 16 bit word in the data stream, with five bits for the channel number, ten bits for the time, and a single bit to indicate the direction of the transition (up or down). Two transitions are packed into a 40 bit output word, the remainder of the word ( 8 bits) is occupied by error flags.

A complete model of the design was constructed in software and simulated on a SUN workstation for functional correctness using DUMAND II Monte Carlo simulation data. The lengths of the FIFO buffers were optimized through these simulations. Simulated events were chosen which resulted in worst-case data streams for digitizer queuing; that is, events where large numbers of PMT pulses arrive simultaneously at the digitizer.

The detailed logic design was developed on a Mentor Graphic CAE system, and verified for correct operation and correct timing using Mentor's Quicksim logic simulator. Vitesse provided a family of macros which were compatible with the Mentor system. The final design was quite complex (200,000 transistors or about 66,000 2-input equivalent gates), and required many hours of CPU time on an Apollo DN5500 workstation for a simulation run. The final task in the ASIC design was to develop a set of test vectors to verify that the fabricated devices operate correctly. An eight layer PC board was designed to house the ASIC and associated circuitry. The design was quite complex due to the high frequencies involved. A block diagram of the PC board is shown in Figure D.9. The PMT signals enter the board on optical fibers from the OMs, and are converted to electrical signals by optical receivers. The signals are then carried to the ASIC on impedance-controlled microstrips. The digitized data is then serialized by the Hot-Rod transmitter and sent to shore. The board also contains an interface to the string controller computer, which allows various diagnostic and control functions to be activated from the shore station. The $O M$ receivers may be individually adjusted to compensate for changes in fiber attenuation, or to keep signals from malfunctioning PMTs from overloading the system. Self-test features on the ASIC itself may be activated for remote debugging. 
DUMAND II Nemino Telescope

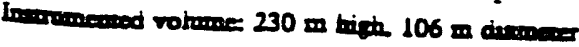

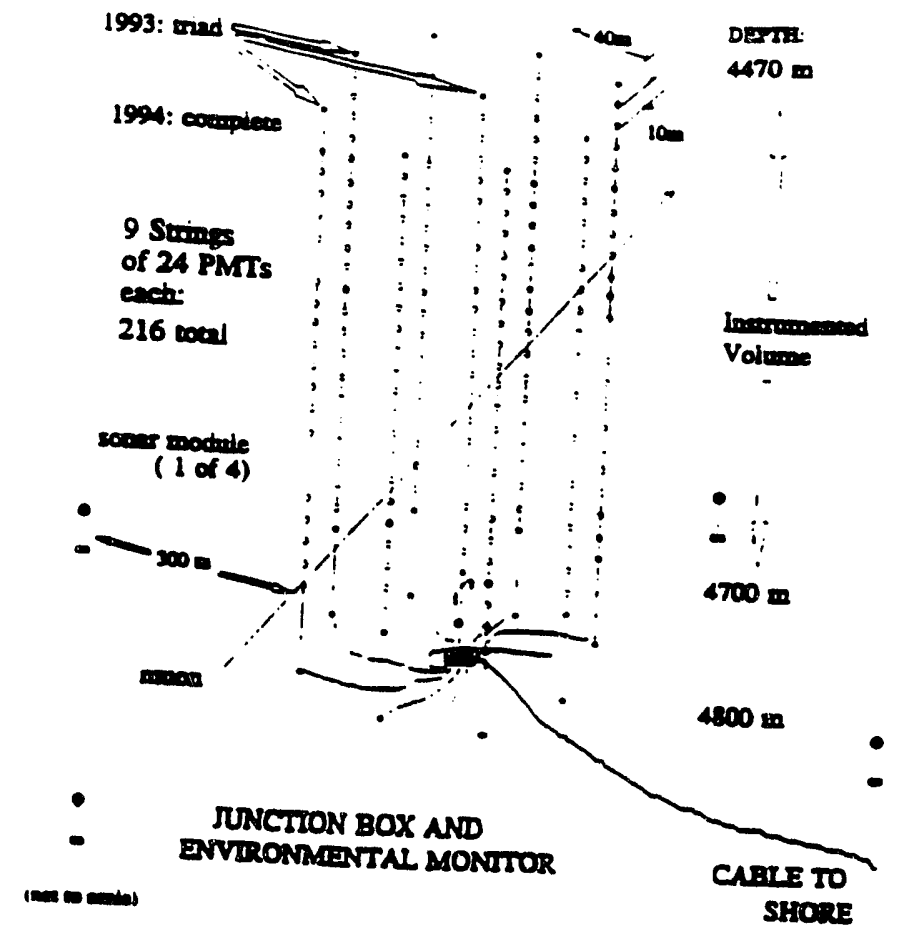

Figure D.7 Schematic representation of the DUMAND II octagon array.

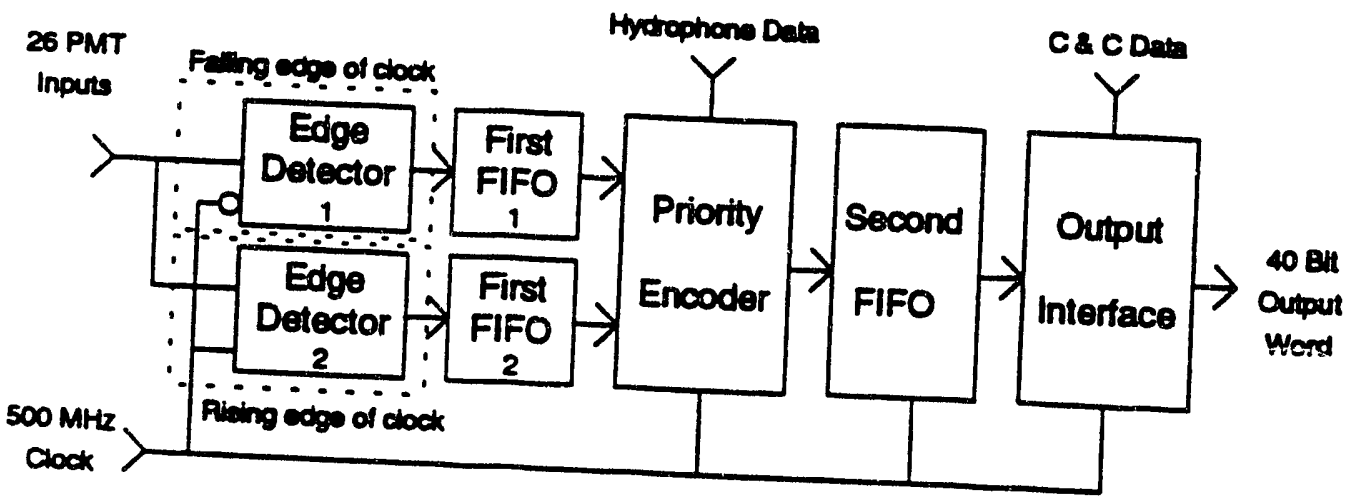

Figure D.8 Block diagram of the DUMAND digitizer ASIC.

Task D: Nextrino Astrophysice and Proton Deeay 


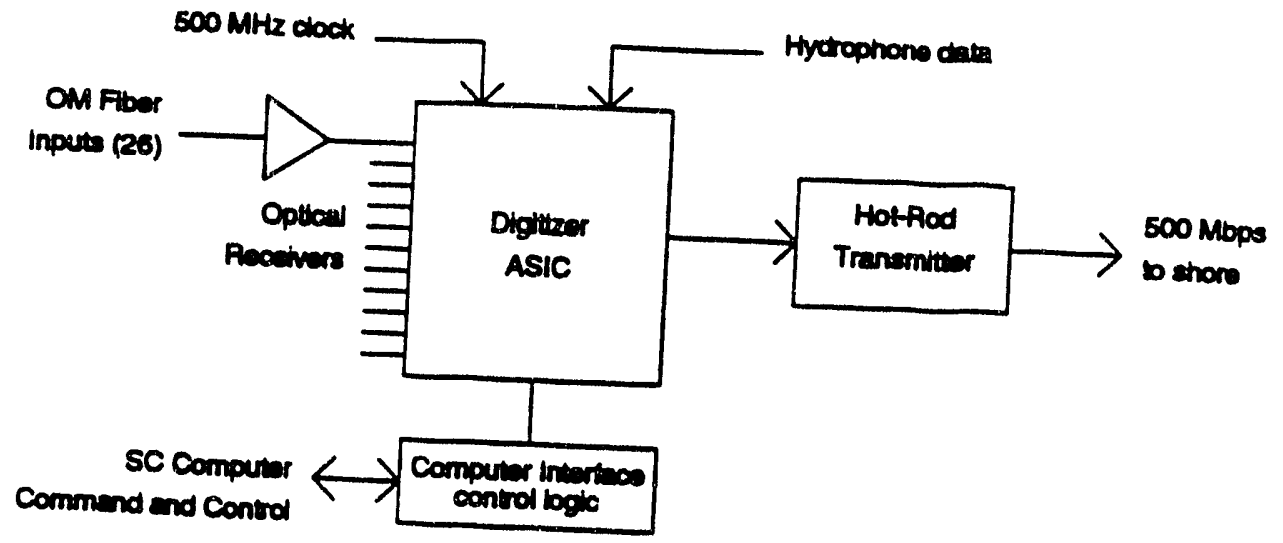

Figure D.9 Block diagram of the DUMAND digitizer PC board.

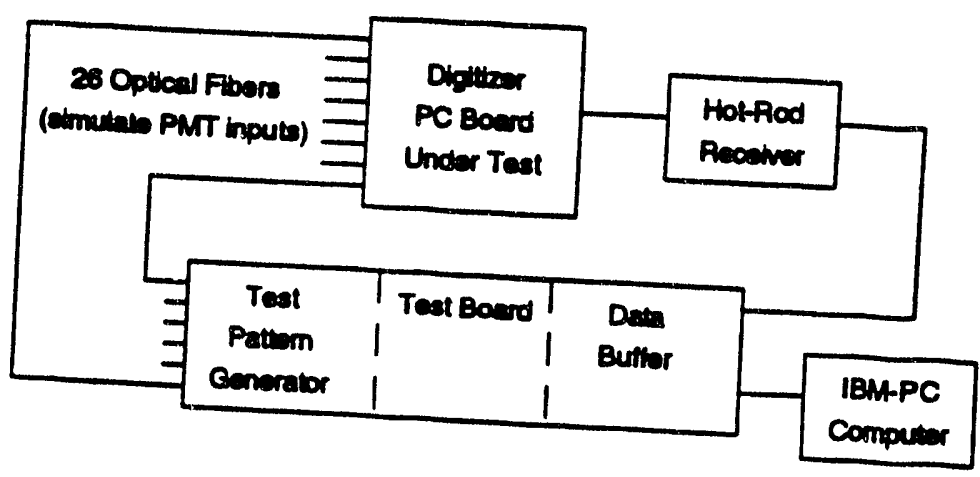

Figure D.10 Block diagram of the DUMAND digitizer test system.
Page s.22 
Preliminary testing of prototype ASICs has shown correct operation at $500 \mathrm{MHz}$. A microprocessor-controlled test setup was designed, as shown in Figure D.10. A set of test patterns is loaded into a 26 bit by $8 \mathrm{~K}$ word memory on the test board. On command the test data is clocked out to 26 optical transmitters to simulate PMT data, and carried to the digitizer board on 26 optical fibers. The output data stream is received by a Hot-Rod receiver, collected in another buffer on the test board, and then transmitted to an IBM-PC for analysis. An extensive testing and burn-in program is planned.

\section{IMB-3 Data Analysis}

More than 2000 neutrino interactions, spanning neutrino energies from $20 \mathrm{MeV}$ to $\sim 1$ $\mathrm{TeV}$, were recorded by the IMB detector. Events are classified by the location of the interaction. Events due to a neutrino interaction inside the detector volume are called "contained events". Contained events are produced by interactions of both $\nu_{\mu}$ and $\nu_{e}$. Because of data selection criteria, contained events are of lower energy ( $<2 \mathrm{GeV})$. Events due to a muon neutrino interaction outside the detector volume are called "external events" or "upward-going muons". These higher energy events enter from below and travel upward. The recorded flux and spectra of both classes of events are consistent with atmospheric neutrinos.

Contained events from a $7.7 \mathrm{kt}$-yr exposure of IMB-3 are used to measure the atmospheric neutrino composition and to estimate proton partial lifetimes. When combined with the 4.3 kt-yr exposure from IMB-1 and IMB-2, sensitivity to proton decay into $e^{+} \pi^{\circ}$ and other lowbackground modes nearly reach the level of $10^{33}$ years. For lower energy modes and those with greater background, the sensitivity is a factor of three to ten less. Data from IMB-1 and 2, plus our preliminary analysis of IMB-3 data, give no compelling evidence that nucleon decay has been observed above background. The atmospheric neutrino composition measurement uses only IMB-3 data. We find an anomalously low fraction of "muon-like" events in the total sample of single ring events. This finding agrees with an independent measurement of the fraction of events with a muon decay signal. Although oscillation of neutrino flavor is a possible explanation of the data, furiher studies are required before this conclusion can be reached.

Over 600 upward-going muon events recorded over the entire working life of the IMB detector are used to search for muon neutrino oscillations. Two methods are employed: 
comparing the measured rate with the expected rate; and the ratio of upward-going muons which stop in the detector to those which exit is used to search for deviations from the expected energy spectrum. This latter technique is free of flux and cross-section normalization uncertainties. No evidence for $\nu_{\mu} \rightarrow \nu_{\tau}$ oscillation is found. $90 \%$ c.l. limits on $\delta m^{2}$ are derived in the range from 1 to $2 \times 10^{-4} \mathrm{eV}^{2}$ for $\sin ^{2} 2 \theta>0.5$.

\section{Nucleon Decay}

There are many philosophical and observational reasons to expect baryon non-conservation. Observations such as the local baryon asymmetry suggest non-conservation and much current theoretical work requires non-conservation at energies near the unification mass. Experimental observation of baryon non-conservation, in the form of nucleon decay, would provide an important clue into the nature of the universe.

For most nucleon decay modes, there is an expected atmospheric neutrino background that must be estimated. Two neutrino interaction models are used to estimate the rate and type of neutrino interactions. There is substantial agreement between the expected and observed events justifying background subtraction.

During a 7.7 kton-year exposure (about $4.7 \times 10^{33}$ nucleon-years) IMB-3 observed 935 contained events. The observed rate and characteristics are consistent with the expected backgrounds from the atmospheric neutrino flux. Partial lifetime limits are set for various nucleon decay modes. Setting nucleon decay rate limits requires selecting candidate events. Since IMB-3 estimates event reconstruction efficiency using simulated data, simulated and real data must be reconstructed with the same efficiency. Care is taken that event selection is not biased toward real or simulated data. IMB-3 relies on many event selection techniques which are easily automated or require substantial hand-fitting by physicists; however, a new multi-segment track fitter (MuSTFit) has been developed which automates many of the functions of hand-fitting. IMB-3 uses several techniques falling into two general categories: topological and physics-based.

The simplest topological parameter used by IMB-3 is anisotropy. Anisotropy is the sum of the directions from the event vertex to each PMT, weighted by the PMT charge. Events with a spherical light distribution, such as a particle decaying at rest into multiple charged particles, have an anisotropy near zero. Single ring events have an anisotropy near one. 
Anisotropy is not a powerful event selection technique, but it is simple. Real and simulated data can be treated identically reducing systematic errors. Unfortunately, different event classes, such as single ring and overlapping double rings, are not well separated. Only events with multiple, non-overlapping rings are unambiguously separated.

In cases where anisotropy is not sufficient to separate event classes, determining the number and direction of visible rings is a powerful tool. This is a difficult task. IMB-3 uses two different methods to determine the number of rings. The first technique, hand-fitting by physicists, is robust, efficient, and can be used on all possible events. Despite the advantages, it is impractical for large data sets such as simulated background data. Systematic errors result from a scanner "knowing" the class of an event, and it is difficult to quantify the definition of hand-scanned rings due to the subjective nature of the decision. The second technique, automated-fitting using MuSTFit, objectively defines rings and has calculable systematic errors; however, MuSTFit is not as efficient as a human scanner.

MuSTFit was developed to overcome the difficulties of hand-fitting. MuSTFit is an automated fitter which requires no human intervention. Events are reconstructed by finding a set of track segments which reproduces the observed light distribution. The track segments are used to estimate the vertex location, the number and the direction of tracks.

IMB-3 uses several physics-based event selection techniques. The simplest techniques are requirements on the total event energy and the number of muon decays, neither of which require substantial post processing to measure. IMB-3 classifies single ring events as resulting from electro-magnetic showers, or non-showering particles. Invariant mass analysis provides a method of background rejection for multiple ring events.

Invariant mass analysis is the most powerful event selection method used by IMB-3 to search for nucleon decay. Analysis proceeds by assuming the identities of decay particles and matching each decay particle to a visible ring. All combinations of particle to ring matches are tried. For every possible hypothesis the invariant mass and initial momentum of the parent particle are calculated. Events with invariant mass and momentum similar to the desired parent particle are selected. The drawback of invariant mass analysis is that the event topology must be previously determined and so suffers from the problems mentioned above. An example of invariant mass analysis is shown in Figure D.11 where the invariant 
mass of two track events with no muon decay are shown. The apparent peak near the $\pi^{\circ}$ mass indicates the high quality of the IMB-3 absolute energy calibration.

Preliminary limits for several modes are given in Table D.1 along with the observed number of events and expected background. All limits use the full IMB-3 exposure, with the exception of $p \rightarrow \nu K^{+}$which uses about half. The background calculation is not complete for $p \rightarrow \nu K^{+}$. There is good agreement between the expected and observed event distributions so background subtraction can be performed. There is a $30 \%$ systematic error in the estimation of the atmospheric background which results from uncertainty in neutrino cross sections and neutrino flux.

The mode $n \rightarrow \nu \pi^{0}$ provides an excellent example of the capabilities of IMB-3. The previous IMB-1 limit had an expected background of 60 events. With the improved light collection of IMB-3 the proper invariant mass can be required for $\pi^{0}$ candidates. Further, the background can be readily estimated with the newly available MuSTFit. Taken in combination, there is an improvement of almost a factor of twenty in the limit for this mode.

\section{Magnetic Monopole Search}

An improved limit on the flux of magnetic monopoles in the vicinity of the solar system is obtained, assuming that monopoles strongly catalyze nucleon decay into charged energetic daughter products (the Rubakov-Callan effect.) Flux limits are obtained for monopole velocities from $10^{-9} c$ to $10^{-1} c$ and for monopole - nucleon cross sections between $10^{-28} \mathrm{~cm}^{2}$ and $10^{-22} \mathrm{~cm}^{2}$. For a representative velocity $\beta \sim 10^{-3}$, and cross-section $\sigma \sim 10^{-23} \mathrm{~cm}^{2}$, we obtain a limit $F_{m}<2.7 \times 10^{-15} \mathrm{~cm}^{-2} s r^{-1} s^{-1}$ at $90 \%$ confidence level.

If magnetic monopoles exist and if they catalyze nucleon decay as suggested by Rubakov, Callan, and others, then proton decay detectors, such as the IMB detector, may be used to set limits on the local flux of monopoles. Monopole-induced nucleon decay is more difficult to detect than spontaneous nucleon decay because the massive monopole can invisibly carry off much of the momentum. This means that the usual searches for nucleon decay, where events with a significant unbalanced momentum are usually interpreted as neutrino-induced background, are not directly applicable. 
Despite their high stopping power $\left(d E / d X \sim 101 \beta \mathrm{GeVg}^{-1} \mathrm{~cm}^{2}\right.$ for $\left.\beta<0.1\right)$. massive monopoles $\left(M \sim 10^{16}\right.$ to $10^{17} \mathrm{GeV}$ for $\mathrm{SU}(5)$ monopoles, in velocities typical of objects in the solar neighbornood $\left(\beta=10^{-4}\right.$ to $\left.10^{-3}\right)$ can still easily reach deep underground detectors such as IMB.

To estimate $\lambda_{c a t}$, the mean distance between catalyzed nucleon decay interactions, we use $\lambda_{\text {eat }}=1 /\left(\sigma_{\text {cat }} N_{N}\right)$, where $N_{N}$ is the nucleon density of the target. Estimates of $\sigma_{\text {eat }}$ are ur furtunately subject to great uncertainty, ranging from about $10^{-26} \mathrm{~cm}^{2}$ to about $10^{-19} \mathrm{~cm}^{\text {? }}$ at $\beta=10^{-4}$. Due to this large range of possible cross ctions, summary listings of monopele flux limits such as should be interpreted with care.

The possible modes of a nucleon decay catalyzed by a monopole interaction are not well known. However, there have been predictions that suggest that modes similar to those of "normai" $S U(5)$ nucleon decay (e.g. $\left.p \rightarrow e^{+} \pi^{0}\right)$ are preferred.

The Parker Bound, $F_{m}<\sim 10^{-15} \mathrm{~cm}^{-2} s r^{-1} s \cdot-1$, a limit based on the survival of the galactic magnetic field, has proven difficult to improve upon by earth-based detectors. More stringent limits can be obtained from catalyzed nucleon decay in pulsars, and from catalyzed nucleon decay in the sun. These indirect astrophysical measurements may be complemente I by more direct measurements of the monopole flux incident on the earth as in the case of large water Čerenkov detectors.

Recent results from the underwater Čerenkov detector at Lake Baikal put the flux limit at $F_{m}<\sim 2 \times 1 J^{-16} \mathrm{~cm}^{-2} s r^{-1} s-1$ for $\beta_{m}=10^{-4}$. However, to achieve this limit, it was necessary io assume enhanced catalysis cross sections, $\sigma_{c a t}=10^{-21} \mathrm{~cm}^{2}$ at this velocity. The Kamiokande detector, a 3000 ton water Cerenkov detector located in Kamioka, Japan, has also reported flux limits based on the observation of monopole-catalyzed nucleon decay. These limits are $F_{m}<\sim 2 \times 10^{-15} \mathrm{~cm}^{-2} \mathrm{si}^{-1} \mathrm{~s}^{-1}$ for $\beta_{m}=10^{-4}$ and $\sigma_{c a t}=10^{-25} \mathrm{~cm}^{2}$.

In 1983, the IMB collaboration published a monopole flux limit $F_{m} \leq 7.2 \times 10^{-15} \mathrm{~cm}^{-2} \mathrm{sr}^{-1}$ $s^{-1}$ at velocities $\beta_{m}=10^{-4}$ to $10^{-1}$, assuming a catalysis cross-section $\sigma_{\text {eat }}=10^{-25} \mathrm{~cm}^{2}$. This limit was derived from the non-observation of multiple nucleon decay events in 100 days of live time. Herein we extend this analysis, with improvements, to another 625 live days of the IMB detector from 4 May 1986 to its shutdown on 22 March 1991. 


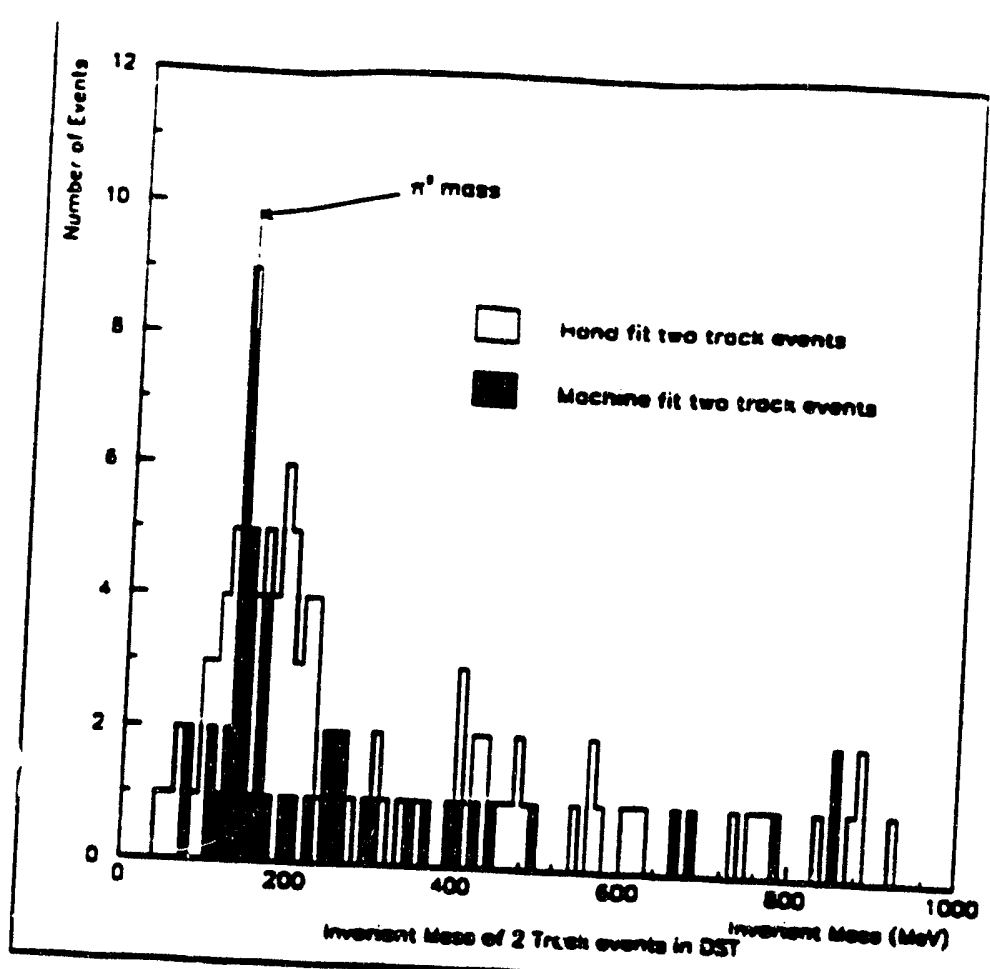

Figure D.11 The invariant mass of two-ring events with no muon decay. The unhashed histogram cuntains all events that were hand-fitted as containing two rings. The hashed histogram contains the subsample of events that were successfully fitted using the multi-
segment track fitter.

\begin{tabular}{|c|c|c|c|c|c|}
\hline$p \rightarrow e^{+} \pi^{U}$ & i Efficiency & $\begin{array}{l}\text { Background } \\
1+(-30 \%)\end{array}$ & $\begin{array}{l}\text { Number of } \\
\text { Evenss }\end{array}$ & IMB-3 Limit & IMB-1+2 Limil \\
\hline $\begin{array}{l}p \rightarrow e^{+} \pi^{u} \\
n \rightarrow \nu \pi^{u}\end{array}$ & $\begin{array}{l}0.48 \\
0.22\end{array}$ & $<0.2$ & 0 & $5.4 \times 10^{32} 1.2$ & $3.1 \times 10^{32}$ \\
\hline$p \rightarrow \mu^{-} \pi^{\prime \prime}$ & $\begin{array}{l}0.22 \\
0.22\end{array}$ & 2.3 & 2 & $1.3 \times 10^{323}$ & $7 \times 10^{30}$ \\
\hline$p \rightarrow \nu K^{+}$ & 0.11 & $\begin{array}{c}<0.2 \\
-\end{array}$ & 0 & $2.5 \times 10^{32} 3$ & $2.7 \times 10^{32}$ \\
\hline$n \rightarrow e^{-} e^{-} \nu$ & 0.45 & 5.0 & 3 & $6.2 \times 10^{31} 1.2$ & $6.2 \times 10^{31}$ \\
\hline$p \rightarrow e^{-} e^{-} e^{-}$ & 0.88 & $<0.2$ & $\begin{array}{l}10 \\
0\end{array}$ & $\begin{array}{l}9.0 \times 10^{31} 1 \\
9.9 \times 10^{32} 1 \\
\end{array}$ & $\begin{array}{l}4.5 \times 10^{31} \\
5.1 \times 10^{32}\end{array}$ \\
\hline
\end{tabular}

Table D.1 Preliminary 90\% C.L. nucleon decay limits. Background is not calculated for all modes. The methods used to select events are: 1) energy versus anisotropy, 2) invariant mass using hand-scanned fits, 3 ) invariant mass using MuSTFit.

Page s. 20

Boston Universuly 
For every event in IMB-3 the light deposition and timing for each phototube is recorded. This information is recorded for 512 ns with 1 ns resolution - a period of time called the $T_{1}$ scale. Immediately following the $T_{1}$ scale is the $T_{2}$ scale, wherein hit times and pulse heights are recorded for all the tubes for an additional $7.6 \mu$ s with 15 ns resolution. The IMB-3 data is divided into a period when most high light level events were not recorded, and a period when all events were recorded.

In the first period, 627 days, most (but not all) of the events with more than 900 photomultiplier hits on the $T_{1}$ scale were not recorded because they were over the maximum light level expected for a single nucleon decay. This limits our sensitivity to multiple catalyzed nucleon decays during this period. The "multi-hit" analysis uses 401 days of data from this period. For the "single-hit" analysis, all 627 days of this data were used. In the second period, 224 live days, all events were recorded and all live time was used in the single-hit and multi-hit analyses. Hence, the total livetimes are 851 days for the single-hit, and 625 days for the multi-hit analyses.

The number of events on the $T_{1}$ scale that are contained within the detector can be used to set limits on the flux of monopoles in the detector. This is our single-hit analysis. The background to a single-hit nucleon decay signal is atmospheric neviśnos that interact in the detector. A flux model for atmospheric neutrinos and a Monte-Carlo simulation of neutrino interactions in the detector were used to determine the expected number of contained events which would survive the data analysis process. The rate of events due to atmospheric neutrinos expected in a fiducial volume that is two meters inside the walls of the detector is $1.00 \pm 0.21$ events/day, with the quoted error reflecting the disagreement in the atmospheric neutrino flux models.

The number of events found in 851 live days is 935 , which corresponds to a rate of $1.10 \pm 0.078$ events/day. The measured excess rate of $0.10 \pm 0.22$ events/day restricts the maximum nucleon decay rate to $R_{m}<0.39$ events/day at $90 \%$ confidence level. From this we can derive a flux limit using the formula

$$
F_{m}<R_{m} /\left(4 \pi A \varepsilon_{1} \varepsilon_{2}\right)
$$


where $A$ is the effective cross section of the detector, $4 \times 10^{6} \mathrm{~cm}^{2}$ and $\varepsilon_{1}$ is the efficiency for scanners to find a monopole interaction event, conservatively estimated by trials with MonteCarlo events as being $90 \%$. The quantity $\varepsilon_{2}$ is the efficiency for a monopole entering the detector volume to produce an event that will pass the analysis selection criteria: an event will be kept by the analysis if it is within the fiducial volume ( 2 meters inside the walls) and if the number of tubes hit on the $T_{1}$ scale is less than 900 . The probability that a monopole will satisfy these requirements was determined by using a simulation of an isotropic flux of monopoles passing through the detector. This probability depends on the velocity of the monopole, the catalysis cross section and, to a lesser extent, the amount of light produced by a monopole induced nucleon decay. Efficiencies for this analysis to detect a monopole that enters the detector reach a maximum of $76 \%$ at low velocities and high cross sections. The resulting flux limits are presented in Figure D.12.

Rather than parameterize the results in terms of velocity dependent cross-sections, the curves are presented for different constant values of $\sigma_{\text {cat }}$, the catalysis cross-section. Even though one does not expect monopole velocities < 10-5 (the Earth's velocity around the sun is $\beta \sim 10^{-4}$ ), we present results for the full range of velocities accessible to our analysis.

Sensitivity to high cross section, high velocity monopoles is reduced because of the large likelihood of multiple monopole interactions on the $T_{1}$ scale which would cause an event to exceed the 900 tube cutoff. The data examined above includes only events that light 70 or more tubes on the $T_{1}$ scale. Monte-Carlo simulations of the preferred and "standard" $\mathrm{SU}(5)$ modes $\left(M+p \rightarrow e^{+} \pi^{0}, M+p \rightarrow e^{+} \eta, M+p \rightarrow e^{+} \rho, M+p \rightarrow e^{+} \omega, M+p \rightarrow \mu^{+} \omega, M+n \rightarrow \nu \pi^{0}\right.$, $\left.M+n \rightarrow e^{+} \pi^{-}\right)$indicate that these reactions almost always produce events with 70 or more tube hits. However, if other modes that produce less Čerenkov light are favored, these flux limits do not apply.

The observation of consecutive interactions of a monopole in the detector can provide a more sensitive technique for detection of monopoles. An interaction inside the detector on the $T_{1}$ scale followed by an interaction on the $T_{2}$ scale or an interaction on the $T_{1}$ scale followed by another detector trigger within $15 \mathrm{~ms}$ (the approximate time of flight across the detector for a monopole with $\beta=10^{-5}$ ) is an indication that a monopole (or some other particle that produces multiple interactions) is present. Two types of multi-hit analysis are available. The search for monopoles with at least one interaction on the $T_{2}$ scale will be 
called the "T $T_{1} T_{2}$ analysis" while the search for monopoles that interact on consecutive $T_{1}$ scales will be called the " $T_{1} T_{1}$ analysis."

The $T_{1} T_{2}$ analysis looks for events in which the number of tube hits exceeds the background level on both the $T_{1}$ and $T_{2}$ scales. The background on the $T_{1}$ scale is negligible, but it is substantial on the $T_{2}$ scale because of phototube afterpulsing, an effect that creates false hits. If the number of tubes hit on the $T_{2}$ scale exceeds the afterpulsing level by an empirically determined margin the event is kept. The effect of this criterion, which is small for the preferred nucleon decay modes, and of all other selection criteria will be accounted for in the detection efficiency.

The events that pass this criterion are then checked to see if they are entering muons. Cosmic ray muons are a significant background to this analysis because a muon event followed by the muon's decay can simulate two monopole interactions.

To reject these muons, events that trigger more that 900 tubes within a 100 ns window on the $T_{1}$ scale and trigger fewer than 70 tubes in any $100 \mathrm{~ns}$ window on the $T_{2}$ scale are eliminated. The $100 \mathrm{~ns}$ window is chosen so that all of the light generated by a muon crossing the detector will be included. Monopole-catalyzed nucleon decay would produce events that have less than $938 \mathrm{MeV}$ of visible energy which almost always results in fewer than 900 tubes hit in the detector (at least $99 \%$ of the time for any mode). Therefore, only monopoles that interact more than once within 100 ns could be lost by this criterion. The second part of this criterion assures that events with more than 70 tubes hit within $100 \mathrm{~ns}$ on the $T_{2}$ scale will be kept. Monopole-catalyzed nucleon decay would almost always result in events with more than 70 tubes hit (as determined by Monte-Carlo simulation), so the efficiency of this procedure for keeping monopole events is high (about $94 \%$ for the modes listed above.)

Another procedure for rejecting entering muons relies on the use of an automatic event fitter to determine whether or not the event is contained within the detector. If the fitter obtains a position for the event vertex that is within $40 \mathrm{~cm}$ of the detector walls and the fitter returns a high confidence level for that fit then the event could be rejected as entering. However, there must again be 70 or fewer tubes hit in $100 \mathrm{~ns}$ on the $T_{2}$ scale before the event is finally rejected; this retains high cross section monopoles that might otherwise be missed. 
The criteria described above reject all but about one out of every 2000 events. The remaining events were visually-scanned by physicists. The events found to originate inside the detector are consistent with neutrino interactions that produce a muon which subsequently decays. They are inconsistent with monopole interactions because the energy deposition on the $T_{2}$ scale is too low (70 or fewer tubes hit) and are therefore rejected. No candidate monopole interactions are found in the $T_{1} T_{2}$ analysis.

The $T_{1} T_{1}$ analysis looks for multiple monopole interactions inside the detector with at least one hit on each of two successive $T_{1}$ scales within $15 \mathrm{~ms}$ of each other. Events within $3.5 \mathrm{~ms}$ of a previously recorded event will be lost due to data acquisition dead time. A previous search for spontaneous nucleon decay yielded 935 events contained within a fiducial volume that is more than two meters inside the walls of the detector. Of these events, 693 were available for this analysis. The livetime before and after these events was searched for other interactions inside the detector within $15 \mathrm{~ms}$. The events found were visually-scanned by physicists to determine if they were consistent with two consecutive monopole-generated interactions. No pair of events was found with both vertices inside the detector.

From these results the following $90 \%$ confidence level upper limit on the monopole flux can be set:

$$
F_{m}<N_{0} /\left(4 \pi A T \varepsilon_{A} \varepsilon_{B}\right) \text {, }
$$

where $N_{0}$ is upper limit on the number of monopole interactions observed (2.3), $A$ is the effective cross section of the detector $\left(4 \times 10^{6} \mathrm{~cm}^{2}\right), T$ is the live time searched (623 days), and $\varepsilon_{A}$ is the efficiency for the scanners to retain a monopole event. The quantity $\varepsilon_{B}$ is the efficiency for a monopole that enters the detector volume to produce an event that will pass the selection criteria outlined above (the $T_{1} T_{1}$ or the $T_{1} T_{2}$ analysis.) This efficiency was determined by studying a Monte-Carlo simulation of an isotropic flux of monopoles incident upon the detector and subjected to the criteria described above. This efficiency depends on three unknowns: the monopole velocity, the nucleon decay catalysis cross section, and the visible energy produced by the nucleon decay. Although these quantities are not well known, there exist rough predictions of their magntudes. Efficiencies for this analysis to detect a monopole that enters the detector reach as high as $97 \%$ at low velocities and high cross sections. It should be noted that $\varepsilon_{B}$ is quite insensitive to the visible energy of the nucleon decay modes because all tube cuts are well below the light level expected from the anticipated 
modes. No significant change in the value of $\varepsilon_{B}$ is observed in Monte-Carlo simulations of the modes listed above. Reactions with much less light production might correspond to less stringent flux limits.

Figure D.13 shows the flux limits obtained from this analysis, combined with those of an earlier IMB monopole search. Each curve is for a different catalysis cross-section, $\sigma_{\text {cat }}$. The loss in sensitivity for low cross sections at around $\beta=10^{-5}$ is due to a $3.5 \mathrm{~ms}$ electronics dead time between a $T_{2}$ scale and the following $T_{1}$ scale. The highest velocities give poor flux limits becaust the monopole exits the detector before the $T_{2}$ scale is activated, giving no chance for the event to pass the $T_{1} T_{2}$ criterion. High cross sections and high velocities are especially difficult because, for a period of time, most events with more than 900 tubes hit on the $T_{1}$ scale were discarded. The loss in sensitivity for the lowest velocities is due to the average time between interactions being longer than the $T_{2}$ scale or even the $15 \mathrm{~ms}$ maximum time between events of the $T_{1} T_{1}$ analysis. Higher cross sections reduce this effect because shorter interaction lengths result in shorter times between interactions.

Local monopole flux limits have been obtained for various velocities and catalysis cross sections. For a reasonable monopole velocity, $\beta=10^{-3}$, the expected catalysis cross-section is $\sigma_{\text {cat }}=2 / 180.17 \sigma_{0} / \beta^{2} \approx 10^{-24} \mathrm{~cm}^{2}$, taking $\sigma_{0}=10^{-28} \mathrm{~cm}^{2}$. The factor of $2 / 18$ takes into account the predicted suppression of the decay cross-section for oxygen nuclei. The flux limit corresponding to this velocity and cross section is $F_{m}<2.7 \times 10^{-15} \mathrm{~cm}^{-2} \mathrm{sr}^{-1} \mathrm{~s}^{-1}$ at $90 \%$ confidence level.

\section{Search for Astrophysical Sources of Low Energy Neutrinos}

The temporal structure of low energy (30 to $60 \mathrm{MeV}$ ) neutrino interactions within the IMB-3 detector during 863 days of livetime between May 1986 and March 1991 has been analyzed. The neutrino data are consistent with that expected for cosmic ray induced neutrino interactions with no bursts evident (excluding SN1987a). When combined with the 327 effective livedays of IMB-1 data, we place a $90 \%$ C.L. upper limit of $\leq 0.71$ galactic supernovae $\mathrm{yr}^{-1}$. We have also performed a temporal correlation analysis with Gamma Ray Bursts (GRBs) using a subset of this low energy neutrino dataset. No significgant correlations were observed for coincidence windows of 1 minute, 1 hour, or 1 day. 
Neutrinos are thought to be produced in a number of astrophysical phenomena. These neutrinos, if detected, can provide a probe into the fundamental processes occurring at the source. Much of the recent work in neutrino astrophysics has concentrated on the high energy realm ( $>$ few.100 MeV). However, as experience has shown, (e.g., solar neutrinos and SN1987a) there may be much to be gained by a study of low energy neutrinos.

One category of astrophysical objects in which a study of low energy neutrinos may be useful is Gamma Ray Bursts (GRBs). Determination of the sources capable of producing GRBs is one of the great mysteries of astrophysics. Whether through accretion processes, stellar collapse, or particle emission and decay, it is thought that neutrino and gamma ray production may be related in many astrophysical processes. Therefore, it is reasonable to expect neutrino emission to be correlated with a GRB.

The neutrino dataset consists of 118 neutrino events firing between 40 and 70 photomultiplier tubes (PMTs) that were recorded over 863 livedays between May 1986 and March 1991. This corresponds to neutrino energies of roughly 30 to $60 \mathrm{MeV}$. All 8 neutrino events recorded from SN1987a are in this range but are not included in this dataset. Analysis has shown that requiring events to have $\geq 40$ PMTs significantly reduces the contamination from events which are due to cosmic ray muon-induced spallation.

Since the products of low energy neutrino interactions are not well correlated with the incident neutrino direction, evidence for neutrino emission from an astrophysical source comes from the observation of either burst structure in the neutrino data or from temporal correlations made with sources detected by other means.

The search for bursts of neutrinos is performed by counting, for each neutrino in the sample, the number of neutrinos falling within an arrival time window $\pm \Delta t$ around the chosen neutrino's detection time. Since the threshold of IMB was systematically lowered during its 6 year operation, the neutrino detection rate is not constant; therefore, the background is estimated using a Monte Carlo method which relies only on the recorded neutrino data sample and not on atmospheric neutrino production models. The Monte Carlo procedure randomizes each neutrino detection time within \pm 10 days centered around the actual detection time (ensuring the new time is within the detector on-time). The constraint of \pm 10 days is required in case the detector response is not uniform over a period greater than a few weeks. 


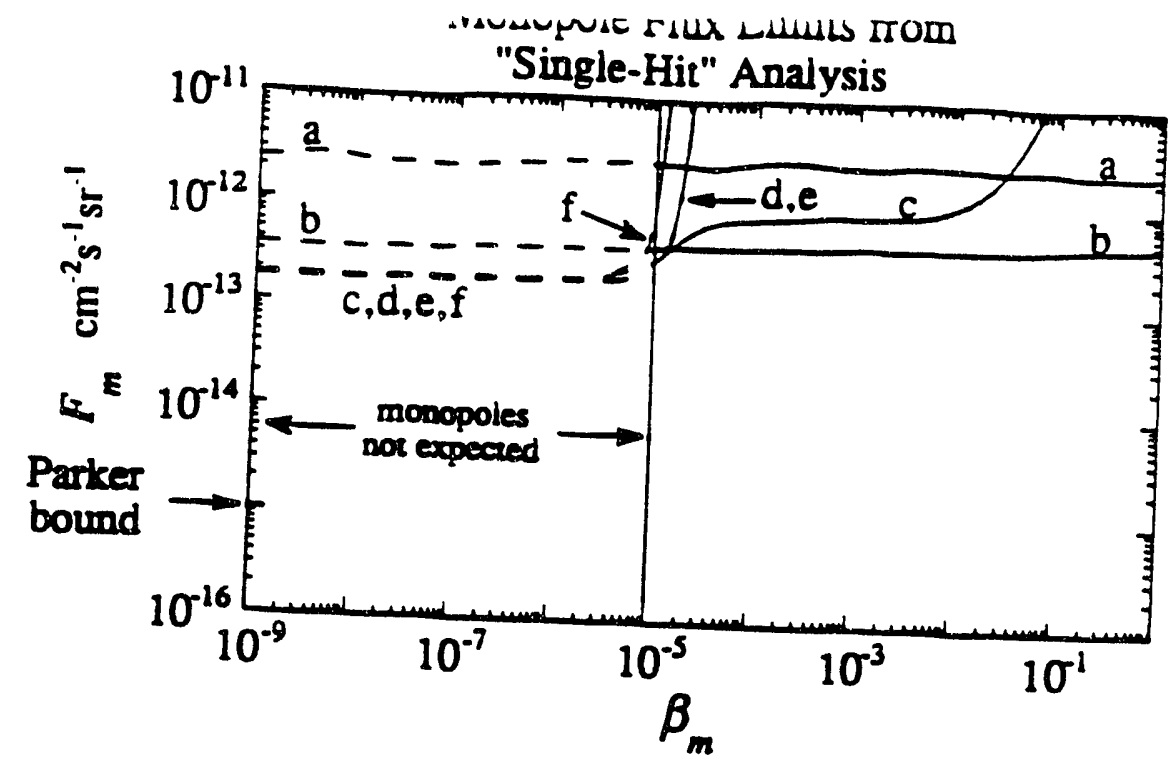

Figure D.12 90\% C.L. flux limits from the single-hit analysis for $\sigma=10^{-28}, 10^{-27}, 10^{-26}, 10^{-25}$, $10^{-24}, 10^{-23} \mathrm{~cm}^{2}$ (curves a through $\mathrm{f}$ ). The Parker Bound for $10^{17} \mathrm{GeV}, \beta<10^{-3}$ monopoles is indicated. Monopole velocities below $\beta \sim 10^{-3}$ are not expected, but the results are presented for their relevance to slow, exotic, light-producing particle searches.

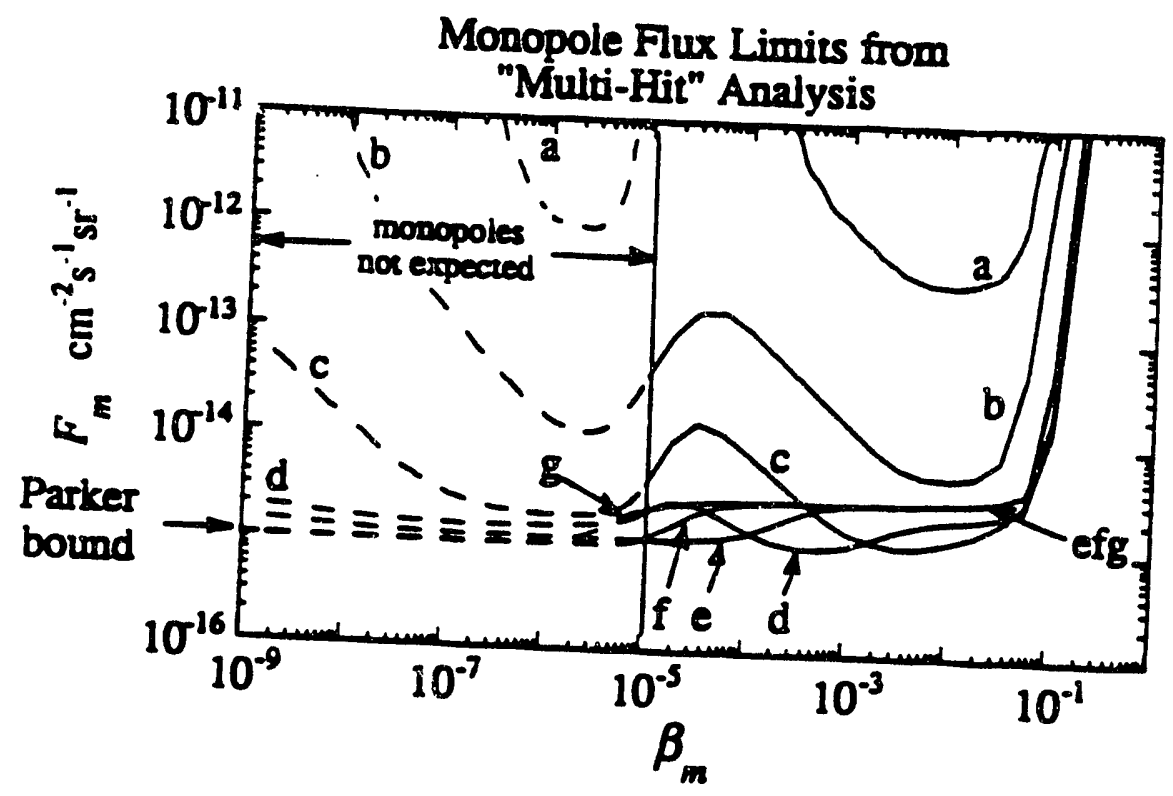

Figure D.13 90\% C.L. flux limits from combined multi-hit analysis and prior IMB results for $\sigma=10^{-28}, 10^{-27}, 10^{-26}, 10^{-25}, 10^{-2 \cdot 4}, 10^{-23}, 10^{-22} \mathrm{~cm}^{2}$ (curves a through $\mathrm{g}$ ). As mentioned in Figure D.12 monopoles with $\beta<10^{-3}$ are not expected, but the results are presented for their relevance to slow, exotic, light-producing particle searches. 
The expected background is the average over $10^{3}$ trials of the number of $\mathrm{n}$-fold coincidences occurring within the arrival time window around each neutrino time. Though consistent with Poisson statistics, this method more accurately reflects livetime and threshold effects.

Table D.2 gives the number of $\mathrm{n}$-fold coincidences between neutrinos in the dataset, along with the expected background $B_{\nu}(\mathrm{n})$, for three different temporal windows. Using the Monte Carlo procedure described above, the chance probability $P_{\geq \text {data }}$, of observing at least as many $n$-fold coincidences as is observed in the data is also given. The significance of the coincidence distribution was determined by comparing the total number of $\geq 1$-fold coincidences with the probability of observing at least that number in the $10^{3}$ Monte Carlo trials. None of the coincidence distributions are statistically significant. Similar background estimates are obtained when the IMB-3 events are randomized \pm 100 days around the actual times or uniformly over the detector livetime because the IMB-3 event rate is essentially uniform for events with $\geq 40$ PMTs.

Standard supernova theory, and our experience with SN1987a, suggests that neutrino emission due to stellar collapse takes place on a timescale of many seconds. Thus, using the applicable 1 minute window we set a $90 \%$ C.L. upper limit on the rate of galactic stellar

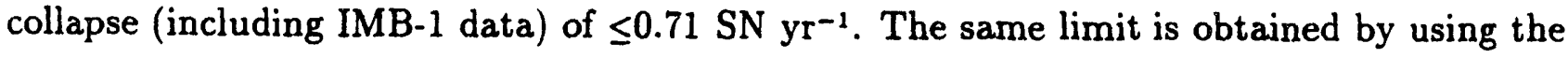
1 hour coincidence window.

The GRB dataset consists of events detected during the IMB-3 livetime. During this period the satellites GINGA and Solar Maximum Mission (SMM) were operational and recorded 53 GRBs. The energetics of mechanisms expected to have correlated neutrino and gamma ray emission (pion production and decay, particle-antiparticle annihilation, etc.) suggest that the low energy neutrino dataset is reasonable for comparison with the gamma ray energies associated with most GRBs (<100 MeV).

The search for coincidences between the detected neutrinos and GRBs is performed by counting the number of neutrinos arriving within a given temporal window around a GRB detection time. The appropriate window is model dependent. If GRBs are the result of stellar collapse-like events then neutrino emission is expected to take place on a timescale of order $\leq 1$ minute. Since IMB records, on average, a cosmic ray induced neutrino in the energy range of interest every 12 calendar days, correlation times longer than a few days would not 
be detectable unless a neutrino burst is detected. Of course some new exotic process may also produce GRBs. These arguments suggest a range of temporal windows: 1 minute, 1 hour and 1 day.

The expected background due to random processes was determined using the same method described above. The IMB-3 events used in this analysis are a subset (115 events) of the total neutrino dataset covering 788 livedays from May 1986 to October 1990 which coincides with operational periods of the satellites. The results of the $\nu / G R B$ correlation analysis are given in Table D.3. None of the coincidence distributions is statistically significant (5\% significance level).

The Monte Carlo method discussed above assumes that the background can be estimated based on the number of neutrinos observed in a window (ie., \pm 10 days) around a GRB detection time. Actually the observed rate of neutrinos is a sample taken from a distribution (assumed Poisson) with an unknown mean. Thus, to confirm the results obtained with the Monte Carlo method, we apply a second method to the correlation analysis which includes the fact that the actual rate of accidentals is unknown.

A method has been developed which involves estimating the probability of Poisson fluctuations of both the observed number of source events and background events. Specifically, this involves calculating the probability of observing $N_{3}$ events or more in the source bin (ie., \pm 1 day around a GRB), given each possible fluctuation in the total number of background events, $N_{B}$. The result is a probability given by

$$
P\left(\geq N_{s}, N_{B}, \alpha\right)=1-\sum_{n_{0}=0}^{N_{0}-1} \frac{\alpha^{n_{s}}}{(1+\alpha)^{N_{B}+n_{0}+1}} \frac{\left(N_{B}+n_{s}\right) !}{N_{B} ! n_{s} !}
$$

where $\alpha$ is the ratio of the source bin size to background bin size. For the coincidence window of \pm 1 day and a background window of \pm 10 days $\alpha=0.1$; however because of livetime effects the actual value of $\alpha$ may be different for a given GRB. The probability given above is computed for each GRB, and the distribution of 53 probabilities is compared to that expected from neutrino events distributed randomly in time. The value of $N_{s}$ expected for each burst from randomly occurring events is estimated by choosing, for each GRB, a random deviate from a Poisson distribution whose mean is $\lambda=N_{B} \cdot \alpha$. The two probability distributions can then be compared using a statistical test developed by Fisher. We define a parameter $\beta$ as 


$$
\beta=-2 \log \prod_{i=1}^{53} \beta_{i}
$$

which for large number of events is distributed as $x^{2}$. This is not the case in this example because of the small number of expected events. Letting the $\beta_{i}$ be the probabilities defined above, we use the Monte Carlo method to determine the significance of the observed coincidences. Performing this calculation for $10^{3}$ estimates of $N$, (ie., number of randomly occurring neutrino events) for each GRB, we find that the statistic obtained with the actual data is not statistically significant ( $\sim 6 \%$ level), consistent with the result based on the Monte' Carlo (see Figure D.14).

Based on the non-observation of any significant $\nu /$ GRB correlations, a model-independent GRB distance lower limit can be derived using

$$
d^{2}=\frac{8 \cdot \eta}{2.3} \cdot d_{0}^{2}
$$

where $d_{0}=0.055 \mathrm{Mpc}$, the distance to SN1987a. The factor of 8 is the neutrino yield in IMB from SN1987a (discussed below). The parameter $\eta$ is a scaling factor which represents the GRB source's neutrino luminosity and spectrum, as compared to that of SN1987a, thus leading to a different neutrino yield in IMB. Thus the GRB distance lower limit is

$$
d=103 \sqrt{\eta} k p c
$$

If the GRB dataset is assumed to be a homogeneous sample then model-dependent limits can be set. Assuming that all the GRBs produce neutrinos with the same luminosity and temperature distribution as SN1987a, we can use the product of 53 Poisson probabilities to place a $90 \%$ C.L. upper limit of $0.043 \nu$ interactions per GRB. Using this neutrino emission limit, the mean inverse square distance is given by

$$
<1 / d^{2}>=\frac{0.043}{\rho \cdot d_{0}^{2}}
$$

where $\rho$ is the neutrino yield from SN1987a and $d_{0}=0.055 \mathrm{Mpc}$. The relationship of the mean inverse square distance to the source distance depends on the spatial distribution of sources. It is not clear what the actual spatial distribution of GRBs is, but recent data suggests a spherical symmetry. We consider two extremes: an infinitesimally thin spherical 
shell centered on the galactic center and a volume distribution of cosmological origin. For distributions with a maximum radius $r$ the mean inverse square distance is given by

$$
<1 / d^{2}>=3 / r^{2}
$$

for a volume distribution, and

$$
<1 / d^{2}>=\frac{-1}{2 R r} \ln \frac{\left(1-\frac{R}{r}\right)}{\left(1+\frac{R}{r}\right)}
$$

for a shell distribution. The offset of the observer from the center of the shell is given by $R(R=0.0075 \mathrm{Mpc})$. The 8 neutrino events observed in coincidence with SN1987a becomes 12 neutrino interactions within the volume of the IMB detector when corrected for triggering efficiency due to one quarter of the detector being inoperative during the supernova. Without prior knowledge of a supernova, or detection of a burst of several events, the data reduction efficiency for finding the low energy events used in this neutrino dataset is $70 \%$ based on comparisons between independent data analysis chains. Thus the neutrino yield is 8 interactions for a supernova at a distance of $55 \mathrm{kpc}$. After 1988, modifications lowered the detector threshold thus increasing the neutrino yield per supernova; however using the experimentally tested pre-198 eutrino yield for the entire detector livetime gives the most conservative distance limit. - is for stellar collapse-like GRBs $<1 / d^{2}>=1.8 \mathrm{Mpc}^{-2}$ which places a lower limit on the distance of 0.56 (1.3) Mpc for the shell (volume) distribution.

The temporal structure of the IMB-3 low energy neutrino dataset shows no evidence of bursts (excluding SN1987a) with durations $\leq 1$ day during 863 days of livetime between May 1986 and March 1991. When combined with the IMB-1 dataset this leads to a $90 \%$

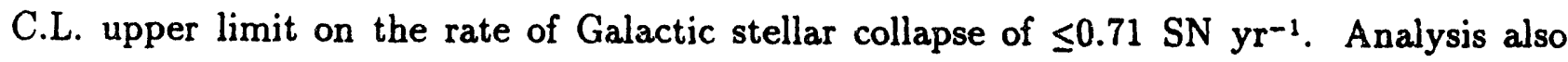
shows no evidence of temporal correlations with GRBs within a coincidence window of $\leq 1$ day, thus allowing a distance lower limit to $i$ set which rules out Galactic halo stellar collapse phenomena as the source of GRBs. Since neutrino and gamma-ray production may be related in many astrophysical processes, it is reasonable to expect neutrino emission from GRBs. We encourage follow-up analysis from other neutrino detectors, especially those that can perform a similar temporal correlation analysis with the BATSE experiment aboard the Compton Gamma Ray Observatory. 


\begin{tabular}{|c||c|c|c||c|c|c||c|c|c||}
\hline \hline \multicolumn{1}{|c||}{$\Delta t=1$ minute } & \multicolumn{3}{c||}{$\Delta t=1$ hour } & \multicolumn{3}{|c||}{$\Delta t=1$ day } \\
\hline $\mathbf{n}$ & Data & $B_{\nu}(\mathrm{n})$ & $P_{>\text {dala }}$ & Data & $B_{\nu}(\mathrm{n})$ & $P_{>\text {data }}$ & Data & $B_{\nu}(\mathrm{n})$ & $P_{>\text {data }}$ \\
\hline 0 & 118 & 117.96 & 0.964 & 118 & 116.56 & 0.236 & 98 & 93.23 & 0.182 \\
1 & 0 & 0.04 & - & 0 & 1.43 & - & 18 & 21.53 & 0.816 \\
2 & 0 & 0.00 & - & 0 & 0.01 & - & 2 & 2.93 & 0.787 \\
3 & 0 & 0.00 & - & 0 & 0.00 & - & 0 & 0.28 & - \\
4 & 0 & 0.00 & - & 0 & 0.00 & - & 0 & 0.03 & - \\
$\geq 5$ & 0 & 0.00 & - & 0 & 0.00 & - & 0 & 0.00 & - \\
\hline
\end{tabular}

Table D.2 n-fold $\nu / \nu$ Coincidences.

\begin{tabular}{|c|c|c|c|c|c|c|c|c|c|}
\hline$n$ & \multicolumn{3}{|c|}{$\Delta t=1$ minute } & \multicolumn{3}{|c|}{$\Delta t=1$ hour } & \multicolumn{3}{|c|}{$\Delta t=1$ day } \\
\hline 0 & $\frac{\operatorname{Dala}}{53}$ & $\frac{B(n)}{5200}$ & $P_{>\text {dale }}$ & Data & $B(n)$ & $P_{\text {>dale }}$ & Data & $B(n)$ & $P_{>\text {d d }}$ \\
\hline 1 & 0 & $\begin{array}{c}52.99 \\
0.01\end{array}$ & 0.988 & 52 & 52.44 & 0.877 & 39 & 43.35 & 0.948 \\
\hline 2 & 0 & $\begin{array}{l}0.01 \\
0.00\end{array}$ & - & 1 & 0.56 & 0.415 & 13 & 8.62 & 0.090 \\
\hline 3 & 0 & 0.00 & - & 0 & 0.00 & - & 0 & 0.95 & . \\
\hline 4 & 0 & 0.00 & - & 0 & 0.00 & - & 1 & 0.07 & 0.061 \\
\hline$\geq 5$ & 0 & 0.00 & - & 0 & 0.00 & - & 0 & 0.01 & - \\
\hline & & & & 0 & 0.00 & - & 0 & 0.00 & - \\
\hline
\end{tabular}

Table D. $\mathrm{n}$-fold $\nu /$ GRB Coincidences.

Page 9.10 


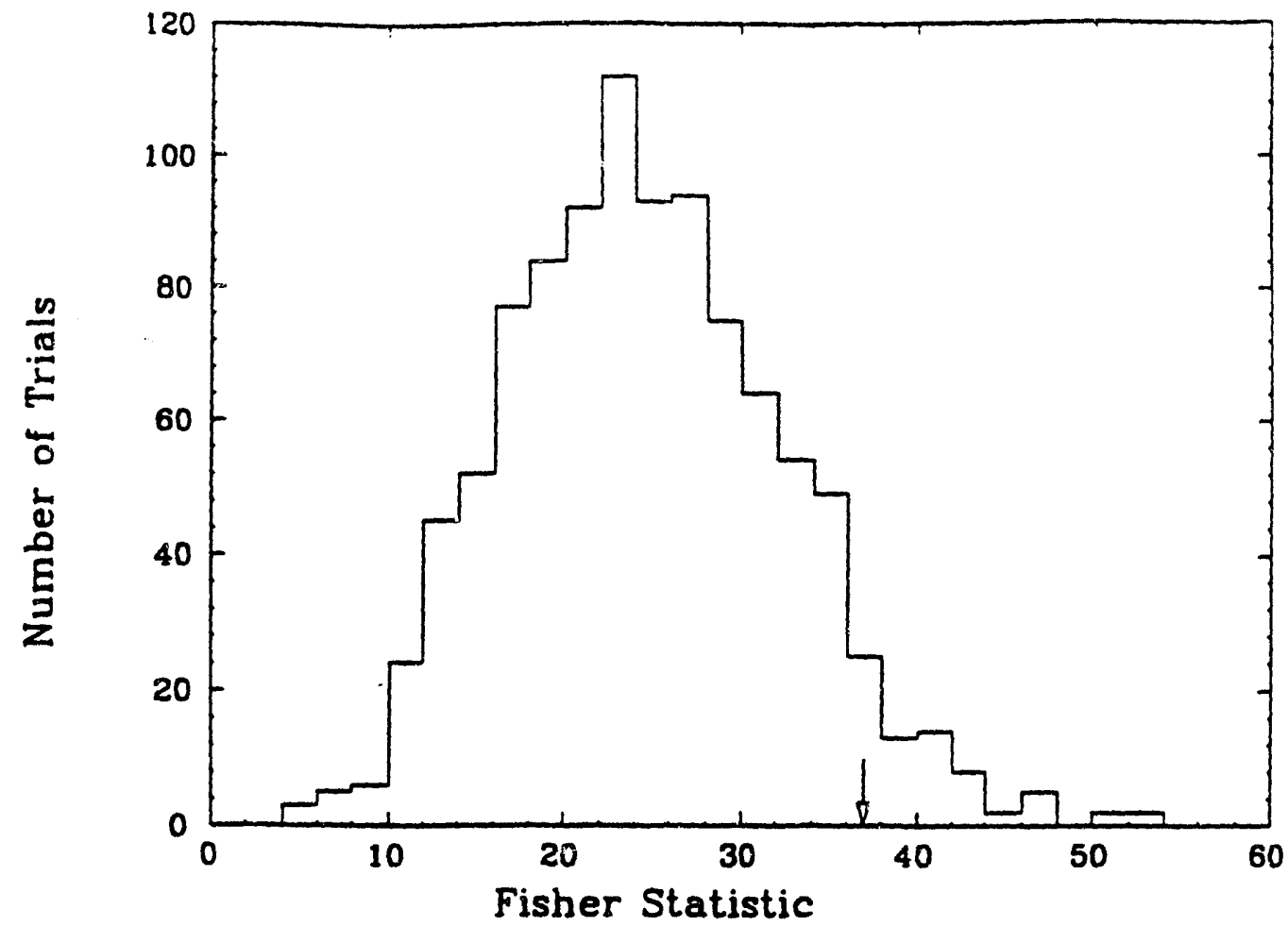

Figure D.14 Fisher statistic distribution for $10^{3}$ trials. The arrow indicates the vaiue of the Fisher statistic obtained with the actual data. Approximately $6 \%$ of the trials exceed this value. 
Operations Budget Discussion

Our successful bid to participate in Super-Kamiokande freshens our enthusiasm to continue the search for nucleon decay. This major new responsibility, the beam test at $K E K$, and forthcoming data from DUMAND justify the proposed budget. We need a second graduate student to fully exploit these exciting opportunities. We request funds for general electronics shop and machine shop costs and miscellaneous supplies to meet our responsibilities for the KEK beam test and Super-Kamiokande construction. Our original operations request did not include support for our participation in the KEK beam test or Super-Kamiokande.

Travel by our group to Japan in the coming year is essential. These trips are required for a number of reasons associated with the new Japanese-U.S. Super-Kamiokande collaboration and the calibration beam test at KEK. These include data-taking shifts at KEK, disassembly and storage of the IMB apparatus at KEK, collaboration meetings, engineering design sessions for the Super-Kamiokande trigger system, etc. In addition, we note that James Stone of our group serves as co-spokesman (with Henry Sobel, UCI) of the U.S. contingent of the collaboration with the Japanese Kamioka group. In this capacity, he needs to travel to Japan for organization meetings, proposal presentations at KEK, and collaboration meetings. Every effort is made to maximize the work accomplished on each trip and to minimize costs by using KEK housing and other cost-saving techniques as we learn them.

Domestic travel is also important during this period, since the IMB group meets more frequently to organize and coordinate our efforts for the KEK beam test and Super-Kamiokande construction. Travel to foreign and domestic conferences and workshops and DUMAND collaboration meetings is also anticipated. 
Recent Publications of Project Neutrino Astroparticle Physics and Proton Decay

\section{A. Papers Published in Refereed Journals}

1. R. Becker-Szendy et al., "New Limits on Magnetic Monopole Flux from the IMB Proton Decay Detector," submitted to the Physical Review D, July 1993.

2. R.S. Miller et al., "A Search for Astrophysical Sources of Low-Energy Neutrinos Using the IMB Detector," Louisiana State University preprint LSU-HEPA-93-4, submitted to the Astrophysical Journal, May, 1993.

3. R. Becker-Szendy et al., "Study of Underground Muons During the January 1991 Radio Flare of Cygnus X-3," Phys. Rev. D47 (1993) 4203-4205.

4. R. Becker-Szendy et al., "IMB-3: a Large Water Cherenkov Detector for Nucleon Decay and Neutrino Interactions," Nucl. Inst. and Meth. A324 (1993) 363-382.

5. R. Becker-Szendyet al., "Electron- and Muon-Neutrino Content of the Atmospheric Flux," Phys. Rev. D46 (1992) 3720-3724.

6. R. Becker-Szendy et al., "A Search for Muon Neutrino Oscillations with the Irvine-MichiganBrookhaven Detector," Phys. Rev. Lett. 69 (1992) 1010-1013.

\section{B. Papers In Conference Proceedings}

1. R. Becker-Szendy et al., "Recent Limits on Baryon Non-Conservation from the IMB-3 Experiment," submitted to Proceedings of 23rd International Cosmic Ray Conference, Calgary, July, 1993.

2. R. Becker-Szendy et al., "A Search for Neutrino Oscillations Using IMB," submitted to Proceedings of 23rd International Cosmic Ray Conference, Calgary, July, 1993.

3. R. Becker-Szendy et al., "A Search for Point Sources Using Downward-Going Muons in IMB," submitted to Proceedings of 23rd International Cosmic Ray Conference, Calgary, July, 1993.

4. R. Becker-Szendy et al., "Magnetic Monopole Flux Limits from the IMB Proton Decay Detector," submitted to Proceedings of 23rd International Cosmic Ray Conference, valgary, July, 1993.

5. R. Becker-Szendy et al., "A Search for Astrophysical Sources of Low-Energy Neutrinos Using the IMB Detector," Louisiana State University preprint LSU-HEPA-93-4, submitted to Proceedings of 23rd International Cosmic Ray Conference, Calgary, July, 1993.

6. The DUMAND Collaboration: C.M. Alexander et al., "Update on the Status of DUMAND II," DUMAND-1-93 submitted to Proceedings of 23rd International Cosmic Ray Conference, Calgary, July, 1993.

7. The DUMAND Collaboration: C.M. Alexander et al., "Optical Module for DUMAND II Japanese Version," DUMAND-2-93, submitted to Proceedings of 23rd International Cosmic Ray Conference, Calgary, July, 1993.

8. The DUMAND Collaboration: P.C. Bosetti et al., "An Optical Sensor for DUMAND II European Version," DUMAND-3-93, Submitted to Proceedings of 23rd International Cosmic Ray Conference, Calgary, July, 1993. 
9. The DUMAND Collaboration: U. Camarini et al., "Trigger Strategies and Processing for DUMAND," DUMAND-4-93, submitted to Proceedings of 23rd International Cosmic Ray

10. The DUMAND Collaboration: J. Hauptman et al., "On the Detection of UHE Cascade Showers with DUM $\triangle$ ND II," DUMAND-5-93, Submitted to Proceedings of 23rd International Cosmic Ray Conference, Calgary, July, 1993.

11. The DUMAND Collaboration: E.S. Hazen et al., "The DUMAND II Digitizer," DUMAND6-93, submitted to Proceedings of 23rd International Cosmic Ray Conference, Calgary, July,
1993.

12. The DUMAND Collaboration: J.G. Learned et al., "Acoustical Neutrino Detection in DU.

13. The DUMAND Collaboration: A. Okada et al., "Estimate of Downgoing Atmospheric Muon Background Events in DUMAND II," DUMAND-8-93, submitted to Proceedings of 23rd
International Cosmic Ray Conference, Calgary, July, 1993.

14. The DUMAND Collaboration: V.J. Stenger et al., "Capabilities of DUMAND II Phase I - 3 String Array," DUMAND-9-93, submitted to Proceedings of 23rd International Cosmic Ray

15. The DUMAND Collaboration: R.J. Wilkes et al., "The DUMAND II Acoustical Locat-

16. R. Becker-Szendy et al., "Recent Limits on Baryon Non-Conservation from the IMB Experiment," American Physical Society, Division of Particles and Fields, DPF '92, Fermilab,
November, 1992 .

17. R. Becker-Szendy et al., "Search for Neutrino Oscillations in IMB," ibid.

18. R. Becker-Szendy et al., "Can the IMB Atmospheric Muon Neutrino Deficit be Interpreted as Evidence for Proton Decay?" ibid.

19. R. Becker-Szendy et al., "Atmospheric Neutrinos: Results from IMB," Proceedings of the International Symposium on Neutrino Astrophysics, ed. Y. Suzuki (Singapore: World Sci-

20. R. Becker-Szendy et al., "Search for Electron- and Muon-Neutrino Oscillations Using IMB,"
ibid.

21. R. Becker-Szendy et al., "The Electron and Muon Neutrino Content of the Atmospheric Flux," Proceedings of the Workshop on Long-Baseline Neutrino Oscillations, Fermilab, ed. M. Goodman (Batavia, Illinois: Fermi National Accelerator Laboratory 1992), p. 159-170.

22. R. Becker-Szendy et al., "A Search for Muon Neutrino Oscillations with the IMB Detector,"
ibid., p. 177-189.

23. R. Becker-Szendy et al., "A Long Baseline Neutrino Oscillation Experiment Using the IMB

24. A. Okada for the DUMAND Collaboration, "Sensitivity of DUMAND II to AGN Neutrinor:" ICRR-Report-269-92-7, Proceedings of the Workshop on High Energy Neutrino Astrophysics, p. 277-287. 


\section{TASK E: THEORETICAL PARTICLE PHYSICS}

Faculty:

Research Faculty:

Visiting Faculty:

Research Associates:

Graduate Students:
Professor K. Lane

Professor C. Rebbi

Associate Professor S. Y. Pi

Assistant Professor R. S. Chivukula

Assistant Professor A. Cohen

Assistant Professor R. Rohm

Assistant Professor E. H. Simmons

Professor R. Brower

Professor S. L. Glashow, Professor A. De Rújula Professor W. Kerler

P. Fendley, S. Selipsky, Y. Shen

R. Singleton, J. Terning, M. Carreau, M. Dugan, T. Vaughn

G. Amelino-Camelia, B. Balaji, M. Camperi, I. Das Gupta

B. Dobrescu, D. Kominis, V. Koulovassilopoulos,

P. Mavromatis, S. Myint, M. V. Ramana,

R. Strilka

\section{RESEARCH PROGRAM}

During the last year, research in our group was carried out by faculty members R. S. Chivukula, A. Cohen, K. Lane, S. Y. Pi, C. Rebbi, R. Rohm, and R. Brower (Engineering), postdoctoral associates P. Fendley, S. Selipsky, Y. Shen, M. Dugan, ${ }^{*}$ M. Carreau, * and T. Vaughn," as well as several graduate students. Beginning in the fall of ' 93 , E. H. Simmons will join the BU faculty and particle theory group. Simmons (who was a postdoctoral associate and SSC fellow at Harvard) is an expe-it on the theory and phenomenology of electroweak and flavor symmetry breaking and is currently a member of the physics simulation group of the GEM collaboration. Also in fall ' 93 , Selipsky and Fendley

* Dugan, Carreau, and Vaughn have independent (non-DOE) support. 
will be leaving Boston University and will be replaced by J. Terning (currently at Yale) and R. Singleton (currently at MIT).

In the following we review our research over the past several years and describe our goals for the coming year. This work may be broadly classified into the areas of electroweak and flavor symmetry breaking, hadron collider phenomenology, cosmology and astrophysics, new held-theoretic methods and models, and nonperturbative studies of quantum field theories. We emphasize that these divisions are somewhat artificial because there are many points of contact and overlap, such as the signatures for technicolor at hadron colliders, weak-scale baryogenesis, lattice computations of higher-order terms in the QCD chiral Lagrangian, and so on.

The work of Chivukula and Cohen is supported in part by DOE Outstanding Junior Investigator grants. A description of their research is also included in the reports for these OJI grants - Task $\mathrm{J}$ for Cohen and Task $\mathrm{N}$ for Chivukula.

\section{A. Electroweak and Flavor Symmetry Breaking}

The origin of electroweak and flavor symmetry breakdown is a major component of our research program. Much of this work has long been focused on the dynamical scenario for these symmetry breakings - technicolor and extended technicolor (TC and ETC). Other approaches on which we have worked are the composite technicolor standard model and the standard-model Higgs boson. We have been involved in recent studies of preciselymeasured electroweak quantities. Our research also includes investigations of the weak interactions of hadrons.

\section{A.1 Technicolor and Extended Technicolor}

Chivukula and Simmons, with M. Golden (Harvard), considered [1] the constraints that critical dynamics places on models with a top quark condensate or strong ETC. These models require that chiral-symmetry-breaking dynamics at a high energy scale plays a significant role in electroweak symmetry breaking. In order for there to be a large hierarchy between the scale of the high energy dynamics and the weak scale, the high energy theory must have a second order chiral phuse transition. If the transition is second order, then close to the transition the theory may be described in terms of a low-energy effective Lagrangian with composite "Higgs" scalars. However, scalar theories in which there are more than one $\Phi^{4}$ coupling can have a first order phase transition instead, due to 
the Coleman-Weinberg instability. They showed, therefore, that top-condensate or strong ETC theories in which the composite scalars have more than one $\Phi^{4}$ coupling cannot always support a large hierarchy. In particular they concluded that, if the Nambu-Jona-Lasinio model solved in the large- $N_{c}$ limit is a good approximation to the high-energy dynamics, then these models will not produce acceptable electroweak symmetry breaking.

Expanding on previous work with Simmons and S. B. Selipsky [2], Chivukula, Simmons, and Terning (with E. Gates (Chicago)) investigated [3] how a slowly running technicolor coupling affects non-oblique corrections to the $Z b \bar{b}$ vertex from extended technicolor dynamics. They have shown that, for a top quark $100 \mathrm{GeV}$ or heavier, there would be effects detectable with experiments at LEP. In view of recent analyses of LEP data on hadronic $Z$ decays, Simmons and Chivukula (and Howard Georgi of Harvard) plan to determine more precisely just which classes of ETC models produce non-oblique effects large enough for LEP to have already seen.

K. Lane, his student B. Balaji, and M. Golden (Harvard) are investigating the precisely-measured electroweak quantities $S, T$ and $U$ in technicolor theories with a "walking" gauge coupling and, possibly, with a strong extended technicolor interaction. Most existing estimates of $S, T, U$ for technicolor theories have been based on scaling resonance masses and couplings or other parameters from QCD. These estimates generally produce a value of $S$ too large to be accommodated by experiment. However, there are strong reasons to believe that scaling from QCD fails in walking technicolor models and, hence, the conclusions of these calculations may not be relevant to these technicolor models. A few calculations of $S$ and $T$ have attempted to take into account the peculiar dynamics of walking technicolor and have produced results more favorable to technicolor. However, because of the complexities associated with walking gauge theory dynamics, these calculations are rudimentary and probably should be regarded as preliminary. Lane, Balaji and Golden hope to develop a scheme for estimating $S, T$ and $U$ that captures the relevant dynamical peculiarities of walking technicolor and, then, to determine whether $S$ and the other parameters remain unacceptably large in this class of theories.

Terning and Appelquist (Yale) are presently working on constructing realistic extended technicolor models, which can be tested by their predictions for the spectrum of quarks and leptons. Such models may also give rise to interesting flavor-changing top quark decays, which might be an observable signal at the Tevatron. 


\section{A.2 Higgs Phenomenology}

Chivukula and a student, D. Kominis, examined [4] perturbatively two-Higgs-doublet extensions of the standard model in the context of the suspected triviality of theories with fundamental scalars. Requiring the model to define a consistent effective theory for scales below a cutoff of $2 \pi$ times the largest mass of the problem, as motivated by lattice investigations of the one-Higgs-doublet model, they obtained combined bounds for the parameters of the model. They found upper limits of $470 \mathrm{GeV}$ for the mass of the light $C P$-even neutral scalar and $650-700 \mathrm{GeV}$ for the other scalar masses.

With another student, V. Koulovassilopoulos, Chivukula investigated [5] the properties of a non-standard Higgs boson. Because of the triviality of the usual scalar symmetry breaking sector, if the Higgs mass is approximately $600 \mathrm{GeV}$ or higher, there must be additional dynamics at a scale $\Lambda$ which is less than a few $\mathrm{TeV}$. In this case the properties of the Higgs resonance can differ substantially from those predicted by the standard model. They constructed a chiral Lagrangian description of a theory with a non-standard Higgs boson and analyzed the features of a theory with such a resonance coupled to the Goldstone bosons of the breaking of $S U(2) \times U(1)$. The effective Lagrangian they constructed describes the most general theory in which the Higgs and the Goldstone bosons are the only particles with a mass small compared to $\Lambda$. They computed the leading chiral corrections to the decay width of the Higgs boson and the contribution to the Peskin-Takeuchi parameter $S$ and presented results for the corrections to Goldstone boson scattering. A longer paper with a more detailed description of the calculations and an analysis of a specific model is in preparation.

\section{A.9 QCD with Many Light Flavors}

With Golden and Dugan, Chivukula [6] investigated the limits of chiral perturbation theory in a QCD-like theory with many light flavors. The chiral Lagrangian for Goldstone boson scattering is a power series expansion in numbers of derivatives. Each successive term is suppressed by powers of a scale, $\Lambda_{\chi}$, which must be less than of order $4 \pi f / \sqrt{N}$ where $f$ is the Goldstone boson decay constant and $N$ is the number of flavors. The chiral expansion therefore breaks down at or below $4 \pi f / \sqrt{N}$. They argued that the breakdown of the chiral expansion is associated with the appearance of physical states other than 
Goldstone bosons and speculated that, since the bound on $\Lambda_{\chi}$ falls as $N$ increases, the masses of resonances will decrease relative to $f_{\pi}$ at least as fast as $1 / \sqrt{N}$.

Expanding on these points, Chivukula (also with Golden and Dugan) re-evaluated [7] the calculations of radiative corrections in technicolor theories. Radiative corrections to electroweak parameters in technicolor theories may be evaluated by one of two techniques: either one estimates spectral function integrals using scaled QCD data, or one uses naive dimensional analysis with a chiral Lagrangian. The former yields corrections to electroweak parameters proportional to the number of flavors and the number of colors, while the latter is proportional to the number of flavors squared anc is independent of the number of colors. They resolved this apparent contradiction by showing that the spectrum of technicolor one obtains by scaling $Q C D$ data to high energies is unlikely to resemble that of an actual technicolor theory. The resonances are likely to be much lighter than naively supposed and the radiative corrections to electroweak parameters may by much larger. They also argued that much less is known about the spectrum and the radiative corrections in technicolor than was previously believed.

\section{A.4 Weak Interactions of Hadrons}

With G. Ecker and A. Pich, Cohen has completed studies of the question of nonanalytic corrections to chiral perturbation theory in radiative kaon decays [8] for which there had been a discrepancy between theory and experiment [9][10]. Given a low energy effective Lagrangian describing the interactions of Goldstone (or pseudo-Goldstone) bosons, chiral perturbation theory is a simple technique for constructing Green functions as a power series expansion in powers of momenta (or symmetry breaking masses) over a scale which is typically $4 \pi f_{\pi}$. There are well-known corrections to this analytic expansion, known as chiral logarithms. These non-analytic terms are determined by unitarity, and are most easily computed by extracting the singular parts of Feynman diagrams constructed from the analytic expansion described above. In a chiral-perturbative expansion, these logarithms are small corrections to the leading terms. Cohen noted that there are circumstances where the non-analytic terms simulate the behavior of poles rather than logarithms, and these pole terms can be larger than the leading analytic parts. The contribution of this pole term may be computed for processes where it is dominant, and yields predictions for certain amplitudes in terms of other measured quantities. An example is 
$K_{L} \rightarrow \pi^{0} \gamma \gamma$, which may be related to the measured $K_{L} \rightarrow \pi^{0} \pi^{+} \pi^{-}$amplitude. Ecker. Pich and Cohen have used this idea to improve the theoretical relation between the experimental $K_{L} \rightarrow 3 \pi$ amplitude and the rate and spectrum for $K_{L} \rightarrow \pi^{0} \gamma \gamma$. The result is in quite good agreement, and resolves the discrepancy between the experimental data and previous theoretical calculations.

\section{A.5 Electroweak Processes at One-Loop}

Selipsky, with B. W. Lynn (Stanford) and D. Levinthal (Florida State), has investigated the ingredients for generalized electroweak self-energy functions from four-fermion processes. The work describes a gauge-invariant redefinition of the $S U(2) \times U(1)$ DysonSchwinger equations, leading to quantities suitable for use in the running couplings and masses of a general radiative-correction effective Lagrangian.

\section{B. Hadron Collider Phenomenology}

It is very likely that the high energy and luminosity of the SSC will be needed to discover and study the physics of electroweak and flavor symmetry breaking. We know that the scale of electroweak symmetry breaking lies near $1 \mathrm{TeV}$, within reach of the SSC. We do not know the scale of flavor symmetry breaking. But several popılar ideas related to or explaining flavor physics - standard or nonstandard (possibly supersymmetric) Higgs bosons, extended technicolor, and some scenarios for quark/lepton substructure - are also within the SSC's range. Here we describe our recent involvement in more detailed studies of this physics at hadron colliders.

Chivukula, Lane and Simmons have been actively involved in hadron collider studies. They continue to collaborate with M. Dugan (Harvard and BU), M. Golden (Harvard) and L. Randall (MIT). For the past two years, Lane was (with Frank Paige, SSCL) the co-leader of the GEM Detectcr Collaboraticn's Physics Group. During the past year, Lane was the Lead Author responsible for the preparation of the Physics Chapter of the GEM Technical Design Report (TDR). This work is described below. Simmons and Golden contributed to the research reported in the GEM TDR Physics Chapter. Chivukula (with Paige and A. Mariohar (UCSD)) organized a workshop on "Electroweak Physics at the TeV Scale," held at the Aspen Center for Physics in July 1993. 


\section{B.1 Physics Capabilities of the GEM Detector}

Lane spent the past year on the preparation of the GEM TDR [11]. As co-leader of the GEM Physics Group, he helped organize the simulations of the GEM detector's response to a variety of physics processes. As Lead Author of the TDR Physics Chapter, he was responsible for getting the simulations completed on time, coordinating input from and feedback to detector subsystem groups, and writing up the simulation results.

The major sections of the TDR Physics Chapter and the people who carried out the work are listed below:

1. Development of the simulation program gemfast for rapid modeling of the GEM detector was carried out by T. Skwarnicki, SMU, with input from M. Brooks (LANL), H. Ma (BNL), S. McKee (Michigan), T. Wenaus (LLNL) and B. Zhou (Boston). This extensive program, based on detailed GEANT simulations of the GEM Baseline design, was critically important for determining the respone of the detector to physics signals and backgrounds. In the few instances that gemfast was insufficiently detailed for simulations (e.g., background rejection for $H^{0} \rightarrow \gamma \gamma$ ), full GEANT simulations were performed. In addition, in several instances gemfast simulations were cross-checked with a full GEANT simulation of the GEM Baseline known as SIGEM. Trigger rates and acceptances were modeled by H. Uijterwaal (SSCL).

2. Higge physics concentrated on signal and background rates for the boson $H^{0}$ of the standard minimal (one-doublet) model. Higgs hoson masses in the range $M_{H}=$ $80-800 \mathrm{GeV}$ were considered. These studies were done by a group from Caltech, led by R. -Y. Zhu and including H. Yamamoto, S. Mrenna, X. Shi and S. Schevchenko. GEM can discover the standard neutral Higgs boson, if it exists, for any mass in the range between the LEP II limit of about $80 \mathrm{GeV}$ up to above the triviality limit of about $700 \mathrm{GeV}$, with an integrated luminosity of $10-30 \mathrm{fb}^{-1}$. The higher integrated luminosity is needed only at the extremes of the mass range of interest: near 80 and near $800 \mathrm{GeV}$. In the intermediate mass region of $80-150 \mathrm{GeV}$, the energy and pointing resolutions of the electromagnetic calorimeter and the vertex finding and $e / \gamma$ separation capabilities of the central tracker are essential for detecting the $H^{0} \rightarrow \gamma \gamma$ signal above the backgrounds. If a signal is fuund, GEN: has the important corr. $_{2}$ iementary capability of being able to confirm the discovery in the associated 
production channel, $H^{0} \rightarrow \ell^{ \pm} \gamma \gamma$. The combined significances for the discovery (in one standard SSC year) of the other intermediate mass Higgs channels, $H^{0} \rightarrow Z Z^{*} \rightarrow$ $\ell^{+} \ell^{-} \ell^{+} \ell^{-}$, are all high except near the cross section minimum at $M_{H} \simeq 170 \mathrm{GeV}$. At the high-mass end, discovery with an integrated luminosity of $10 \mathrm{fb}^{-1}$ requires confirmation of the $\ell^{+} \ell^{-} \ell^{+} \ell^{-}$signal in the $\ell^{+} \ell^{-} \bar{\nu} \nu$ and, possibly, the $\ell^{+} \ell^{-}$jet jet channels. Alternatively, a clear signal can be obtained in the $\ell^{+} \ell^{-} \ell^{+} \ell^{-}$channel within a year at higher luminosity (approximately $100 \mathrm{fb}^{-1}$ ).

3. Two aspects of top-quark physics were studied. Discovery of the top-quark and measurement of its mass using two different modes, $t \rightarrow W^{+} b \rightarrow \ell_{\text {isolated }}^{+}+\mu_{\text {non-isolated }}^{-}+X$ and $t \rightarrow W^{+} b \rightarrow 3$ jets, was investigated by C. Yanagisawa (Stony Brook). The large signal rates permit tight cuts to purify the sample. For a heavy top quark with $200 \mathrm{GeV} \lesssim m_{t} \lesssim 300 \mathrm{GeV}$, the top-quark mass can be determined to a few $\mathrm{GeV}$ with a data sample of $\int \mathcal{L} d t=10 \mathrm{fb}^{-1}$. Systematics dominate the error. The discovery of a charged Higgs-like scalar in the decay $t \rightarrow H^{+} b$ was studied by M. Mohammadi and D. Skzyrniarz (Stony Brook). The charged scalar was assumed to decay as $\mathrm{H}^{+} \rightarrow$ one or three prongs. It was found that the $\mathrm{H}^{+}$could be discovered in this way for a wide range of masses and branching ratios, including much of the interesting parameter range of the minimal supersymmetric standard model (MSSM).

4. Jet physics with GEM was studied by R. Carey (Boston). The principal concern here was whether GEM's nonuniform and non-compensating calorimetry would inhibit the discovery of quark substructure at the scale $\Lambda \simeq 25 \mathrm{TeV}$ by generating a false high- $p_{T}$ excess signal or by hiding a real one. It was shown that energy weighting schemes such as those employed for the $\mathrm{H} 1$ calorimeter may be used to correct jet energies well enough to see the $25 \mathrm{TeV}$ compositeness signal.

5. GEM's capabilities for studying high mass physics at ultra-high luminositj', $\mathcal{L} \simeq$ $10^{34} \mathrm{~cm}^{-2} \mathrm{~s}^{-1}$, were studied by a group led by $\mathrm{K}$. Lane. GEM's robust muon sustem and high-precision electromagnetic calorimetry (augmented by central tracking for electron-momenum pointing) were exploited to distinguish different models of heavy $Z^{\prime}$-bosons and of quark-lepton substructure. S. McKee (Michigan) and Simmons investigated two models of a $4 \mathrm{TeV} Z^{\prime}$ decaying into $e^{+} e^{-}$. They showed that 
it was possible to distinguish the models on the basis of the $Z^{\prime}$ widths. The measured widths agreed with the theoretical values to within the GEM mass resolution of $\Delta M_{e^{+} e^{-}} \cong 0.003 \times M_{e^{+} e^{-}}$. For the models considered, the ultimate reach in $M_{Z^{\prime}}$ for a data sample of $100 \mathrm{fb}^{-1}$ was found to be about $8 \mathrm{TeV}$. M. Mohammadi and W. Orrick (Stony Brook) investigated the same two models of a $4 \mathrm{TeV} Z^{\prime}$ in the decay mode $Z^{\prime} \rightarrow \mu^{+} \mu^{-}$. After considering all physics and instrumental backgrounds (such as neutron and gamma fluences, and muon-generated electromagnetic showers into the muon chambers), they showed that the two models could be distinguished by the angular distribution of the outgoing $\mu^{-}$with respect to the incoming quark direction (defined by the boost rapidity of the heavy $Z^{\prime}$ ). M. Golden (Harvard) and Lane considered two models of quark-lepton substructure at the scale $\Lambda=25 \mathrm{TeV}$ in the mode $\bar{q} q \rightarrow \mu^{+} \mu^{-}$. For a data sample of $100 \mathrm{fb}^{-1}$, muon angular distributions were shown to distinguish easily the chiral structure of the underlying contact interaction. The ultimate reach in substructure scale with such a data sample was estimated to be $30-35 \mathrm{TeV}$.

6. Supersymmetry and missing trans'verse energy $\left(F_{T}\right)$ were studied by $F$. Paige and A. Vanyashin (SSCL). They considered several MSSM parameter sets that cover the range of gluino masses from the Tevatron upper bound of about $300 \mathrm{GeV}$ to the plausible maximum of about $2 \mathrm{TeV}$. In the inclusive $\mathbb{E}_{T}$ cross section, the standardmodel physics background is larger than the signal. Furthermore, the detector-induced background from mismeasured jets in the forward region is several times larger than the real background, even for an ideal calorimeter covering GEM's nominal range of $\eta<5.5$. By selecting events with multiple jets and large transverse sphericity $\left(S_{T}>0.2\right)$, signal-to-background rates in GEM were increased to about 5-10. For $M_{\tilde{g}} \simeq 300 \mathrm{GeV}$ there are $10^{6}-10^{7}$ signal events per $10 \mathrm{fb}^{-1}$, so significance is not an issue. Even for $M_{\bar{g}}=2 \mathrm{TeV}$, there is a signal of several hundred events over a small background after jet multiplicity and $S_{T}$ cuts. Finally, it was shown that the like-sign dilepton signature of gluino pair-production can also be used to discover it up to a mass of $2 \mathrm{TeV}$.

The physics simulations described in the GEM TDR Physics Chapter are an essential part of the optimization of the detector. This optimization is an involved process - from 
simulating the detector design with GEANT and other detailed programs, to parameterizing and encoding the simulations in gemfast, carrying out the simulations of the physics signals and their backgrounds, and feeding the results of the simulations back into the design. Following the extensive round of simulations presented in the TDR, the collaboration initiated a program of design optimization and/or further studies focused on:

1. Improved acceptance of the muon system. This is important both for low-mass, $H^{0} \rightarrow$ $Z Z^{*} \rightarrow \mu^{+} \mu^{-} \mu^{+} \mu^{-}$, and high-mass, $Z^{\prime} \rightarrow \mu^{+} \mu^{-}$, processes.

2. Further reduction of the material in the middle superlayer of the muon system, to improve the resolution in low-mass processes such as $H^{0} \rightarrow Z Z^{*} \rightarrow \mu^{+} \mu^{-} \mu^{+} \mu^{-}$.

3. A detailed alignment procedure, using muons produced in $p p$ collisions, to determine the $x y$-position of the muon spectrometer with respect to the center of the beam spot to within $200 \mu \mathrm{m}$. This constraint would significantly improve the resolution for highmomentum muons, for which measurement in the first superlayer is often lost due to electromagnetic debris.

4. The detailed calibration strategy for the EM calorimeter. While there is considerable experimental evidence confirming the stability and uniformity of liquid ionization calorimeters, it is essential to ensure that the constant term in the energy resolution is kept small. This is important for measurements of $H^{0} \rightarrow \gamma \gamma$ and $Z^{\prime} \rightarrow e^{+} e^{-}$.

5. A careful program of calibration to ensure that the jet energy scale will be wellunderstood. This is important for top and other flavor physics, for $H^{0} \rightarrow Z Z \rightarrow$ $\ell^{+} \ell^{-}$jet jet, and for studies of jets at the highest $E_{T}$.

6. Improvement of the $\mathbb{W}_{T}$ resolution by use of a smaller beam pipe at the position of the forward calorimeter, to further extend the rapidity coverage. Consideration of the neutron and photon backgrounds and realistic clearances are an integral part of the design process.

The GEM TDR was submitted to the SSC Laboratory on April 30 and reviewed by the Program Advisory Committee at the end of May. The PAC's report on the TDR was very favorable. In the case of the analysis of GEM's physics capabilities, the PAC requested only a few minor clarifications and/or additional studies. The PAC review of GEM, originally scheduled to be completed in early August, has been suspended pending the outcome of deliberations on the SSC in Congress. 


\section{B.2 Phenomenology of a "Hidden" Symmetry Breakina Sector}

Expanding on previous work [12], Chivukula (with M. Golden (Harvard), D. Kominis. and M. V. Ramana) calculated [13] the production rate of gauge-boson pairs at the SSC in a model with a "hidden" electroweak symmetry breaking sector. They showed that the signal of electroweak symmetry breaking is lower than the background and that one cannot necessarily rely on gauge boson pairs as a signal of the dynamics of symmetry breaking. These results were also reported [14] at the conference on "High Energy Physics with Colliding Beams" held at Yale University, Oct. 2-3, 1992.

\section{B.9 Proposed Research}

Two years ago, Lane and his student $M$. V. Ramana published a paper detailing the hadron collider phenomenology of so-called multiscale models of walking technicolor [15]. One of the more striking expectations of this phenomenology is that color-octet technirho vector bosons may lie below threshold for decay into pairs of color-octet and color-triplet technipions and, thus, can decay only into quark-antiquark and gluon pairs. These $\rho_{T}^{8}$ would appear as striking narrow resonances in dijet production in hadron collisions. Furthermore, they may be light enough to be produced at the Tevatron collider. Members of the CDF collaboration have searched for these dijet resonances and have not found them. However, the jet energy resolution of the CDF detector is not good enough to have put meaningful limits on the existence on the $\rho_{T}^{8}$ discussed by Lane and Ramana. Following a suggestion by Frank Paige, Lane, Simmons and P. Cho (Caltech) are investigating the possibility that an experimentally accessible signal-to-noise may be had by concentrating on $b \bar{b}$ jet production. They are in contact with a member of CDF on the feasibility of detecting the $b \bar{b}$ signal.

Lane, Simmons and Cho also intend to participate in searches at the Tevatron for the processes $g g, \bar{q} q \rightarrow \rho_{T}^{8} \rightarrow \pi_{T} \pi_{T}$ where the expected technipion decays are $\pi_{T} \rightarrow b \bar{b}$ for color octets and and $\pi_{T} \rightarrow b \tau$ for color triplets. As in the $b \bar{b}$ dijet search, the CDF detector is better suited because of its silicon vertex detector and inner tracking.

As CDF collects more data on hadronic scattering, it becomes possible to detect the presence of non-standard color physics at higher and higher scales. If the non-standard physics originates at energies above those reached directly by the collider, then whatever its actual source (e.g., heavy colored particles, compositeness...), the new physics can be 
parametrized by the coefficients of non-renormalizable operators composed of quark and gluon fields. Simmons and Cho are calculating the effect of the leading non-renormalizable gluon operators on the inclusive single-jet spectrum at Tevatron energies. Preliminary results indicate that published data places a lower bound of order $2 \mathrm{TeV}$ on the scale of such new physics. After finishing this work, Simmons and Cho' plan to explore the possibility of setting limits on exotic color physics using CDF's dijet data as well.

Once high-energy hadron colliders produce large numbers of top quarks, detailed measurements of top quark properties may reveal non-standard interactions related to the quark's large mass. Simmons and Chivukula plan to consider how studies of top quark branching fractions can reveal the presence of exotic physics including charged scalar particles. Simmons and Cho plan to study the effect of non-standard color interactions on top quark production.

Cohen and De Rújula are considering the possibility that experiments at HERA might be able to distinguish the contribution of charm states to the structure function of the proton. Using a combination of perturbative QCD and Regge theory they are attempting to estimate this effect.

\section{Cosmology and Astrophysics}

\section{C.1 Finite Temperature Quantum Field Theory and Phase Transitions in the Early Universe}

$\mathrm{Pi}$ and Cohen have been studying issues in finite temperature quantum field theory, which describes equilibrium and non-equilibrium aspects of phase transitions in the early universe - key ingredients of cosmology.

Cohen is in the process of completing a lengthy project with S. Coleman (Harvard) concerning the proper definition of time dependent quantities in quantum field theory at finite temperature. In particular they have considered the question of the decay of a metastable extended configuration in a weakly coupled field theory. The notion of the decay time of such a configuration is actually quite subtle-a priori it is not even clear that a universal "lifetime" exists. That is, unlike the zero temperature case (or the case of quasiparticles at finite temperature), it is not obvious that there exists an exponential decay law that is measured in an appropriate gedanken experiment, where the exponential 
rate is independent of the way in which the initial state is prepared. Indeed, most attempts at calculations of these quantities have assumed a very particular initial state; the final result for the lifetime may depend sensitively on this initial form. This would not be a disaster, but it would mean that in any calculation one would have to decide precisely what the initial configuration should be (for example, in une early universe the configuration at a phase transition may depend on the past history of the universe).

However, Cohen and Coleman have shown that for a broad class of initial configurations (which include the physically relevant cases) there is indeed a universal exponential decay law corresponding to an observable in an appropriate experimental arrangement. This observable is not what is usually calculated, and indeed the relationship between this observable and that usually calculated is obscure. In addition they have developed a formalism appropriate for semi-classical approximations to the thermal decay time. A manuscript based on this work is currently in preparation. Cohen and Coleman plan to continue their investigations of finite time-temperature effects in weakly coupled field theories. They hope to eventually calculate such rates in complicated field theories such as the standard model.

Pi's recent research concerns the question of higher order finite tem crature corrections to the effective potential. The approximate critical temperature of a given phase transition can be obtained by calculating the one-loop finite temperature effective potential [16]. However, cosmological scenarios often rely on the detailed nature of the phase transition, namely, whether it is of first or second order. A more precise determination of the critical temperature and the nature of the phase transition requires analysis of an infinite series of certain classes of multi-loop diagrams in perturbation theory, which are the leading corrections at finite temperature.

Therefore, what is needed is a self-consistent approximation technique in which a large class of diagrams can be summed. One such technique is the effective action for composite operators formulated by Cornwall, Jackiw and Tomboulis (CJT) [17]. The CJT effective action is a generalization of the ordinary effective action which depends not only on a possible expectation value of the quantum field, but also on a possible expectation value of the propagators. Pi has generalized the CJT technique to finite temperature and observed that the class of diagrams which are the leading contributions to the finite temperature 
effective potential are summed and appear as the non-trivial lowest order term in the CJT effective potential.

$\mathrm{Pi}$ and her student Amelino-Camelia have studied $\lambda \Phi^{4}$ scalar theory at finite temperature using this technique [18]. They have carried out a complete analysis in which all "daisy" and "super-daisy" diagrams, which are the leading high temperature corrections, are included. They have established that in this approximation, the finite temperature effective potential has two degenerate minima for some range of temperatures. However, in $\lambda \Phi^{4}$ theory, the second minimum appears at a value which is not reliable in this leading order approximation. Nevertheless, the result indicates a possibility that the first ordor phase transition may occur in a more realistic model where gauge fields and fermions play an important role.

Although the finite temperature C.JT effective potential can be applied to any field theoretic model, the actual evaluation of the loops in terms of the complete propagators involves technical difficulties. The most important technical issue is how to study the momentum dependence of the effective mass, which arises in the leading order raiculations in gauge theories. $\mathrm{Pi}$ has developed a further approximation to study the momentum dependence of the effective mass using Rayleigh-Ritz type variational techniques [19]. This approximation, unlike the one frequently used in the literature where one makes the ad hoc replacement $M(p) \simeq M(0)$, preserves the self-consistency between the effective potential and the gap equations. In spirit, this method of performing the approximation at the level of the expectation value of the Hamiltonian, rather than at the level of the gap equations that follow from varying the exact Hamiltonian, is very similar to the difference between the Kohn-Sham approximation and the Slater approximation in Hartree-Fock many-body theory. The former is an approximation to the Hamiltonian expectation value, which is then varied; the latter is an approximation to the variational equations. It is known that the Kohn-Sham method is better.

Amelino-Camelia, Pi's student, has studied the finite temperature effective potential in the Abelian Higgs model using the above technique [20]. He finds an interesting result that, unlike in the $\lambda \Phi^{4}$ theory, the location of the second minimum of the effective potential is in the range where the "daisy" and "super-daisy" re-summed approximation is valid.

$\mathrm{Pi}$ and Amelino-Camelia are continuing their analysis for the standard electroweak phase transition. Also, together with Brower, they are performing a numerical study of 
the higher order corrections in $\lambda \Phi^{4}$ theory in order to establish a more reliable description of the phase transition.

\section{C.2 Weak-Scale Baryogenesis}

The question of the origin of the baryon excess of the observable universe is one of the most profound we face. Particle physicists have come to understand some of the ingredients necessary for gencrating the baryon excess: fundamental baryon number non-conservation (either exolicitly or spontaneously broken), CP violation, and out-of-thermal-equilibrium processes [21]. In the late 1970s it was shown how these three ingredients could arise in the context of Grand Unified Theories (GUTs) and lead to a baryon excess in the present epoch [22]. Indeed, the presence of taryon violation and the fact that it leads to a baryon excess today remains one of the most attractive features of GUTs. However, it is difficult to obtain a baryon excess of the size observed today without excessively complicating or fine-tuning the GUT. In addition, the absence of proton decay at the rate predicted by the simplest GUTs has cast some doubt on this explanation of the origin of the baryon excess.

Cohen, D. Kaplan and A. Nelson (UCSD) have used spontaneous baryogenesis to construct models that can produce a baryon asymmetry at the weak phase transition. In Ref. [23] they developed a new method for generating a baryon asymmetry that, rather than avoiding fast weak interaction baryon violation at high temperature, makes direct use of it. The key problems for using weak baryon violation are to maintain departure from thermal equilibrium at the weak scale and to find a source of CP violation to drive baryon violation in the right direction. In one model, the departure from equilibrium was accomplished by having the weak phase transition be weakly first order. The phase transition then occurs through bubble nucleation, with a non-equilibrium separation of phases. Cohen, Kaplan and Nelson applied this idea to a standard model with extra Higgs doublets [24].

Cohen and Nelson have investigated baryogenesis in supersymmetric models [25]. In order for the minimal supersymmetric standard model to produce the observed asymmetry, all of the parameters (such as the gaugino masses, higgsino masses, phases, etc.) must lie within very narrow ranges. This yields a rather definite prediction for the electric dipole moment of the neutron and, with some assumptions about squark and slepton masses, 
for the electric dipole moment of the electron. These constraints can be relaxed in nonminimal models through the addition of gauge singlets which can change the details of the phase transition.

Cohen, Kaplan and Nelson have completed a long review of the subject of electroweak baryogenesis for Annual Review of Nuclear and Particle Science [26]. This review covers the basic concepts of baryon violation in the standard model, details of phase transitions in the early universe with emphasis on the electroweak transition, and a lengthy discussion of the generation of the baryon asymmetry at such a transition.

During the coming year Cohen plans to continue his work on baryogenesis. Recent suggestions by Shaposhnikov and Farrar that the observed baryon asymmetry may arise in the standard model with no new source of CP violation have generated a great deal of controversy. Currently Cohen is investigating these claims and hopes to provide a reliable estimate of the maximum baryon asymmetry produced in this model.

Cohen, Simmons and N. Rius (MIT) are considering how weak-scale baryogenesis may arise in models where the electroweak symmetry is dynamically broken. One possibility for generating the necessary CP violation lies in the "composite technicolor standard models" of electroweak and flavor symmetry breaking.

\section{C.\& Relic Farticles}

Chivukula and Cohen, with M. Luke and M. Savage (both of UCSD), estimated [27] the cross section for the scattering of a slow, color-neutral technibaryon made of colored constituents with nuclei. Contrary to previous estimates [28], they found a cross section of order $A^{2} 10^{-45} \mathrm{~cm}^{2}$, where $A$ is the atomic number of the nucleus. They concluded that, even if technibaryons constitute the dark matter in the galactic halo, this is too small to be detected in future underground detectors.

Chivukula and Selipsky have extended [29] a previous study of stable massive charged particles in the early universe, with implications for new degrees of freedom (such as technicolor) to be sought at the SSC. After the nucleosynthesis era, negatively charged, color-neutral particles would electrostatically bind protons and alpha particles, and appear today as TeV-mass neutral or positively charged nuclei. Obtaining cross sections for the bound states' rate equations requires the Schrödinger equation to be solved numerically; the bound states are not hydrogenic, because the alpha-particle charge radius is comparable to 
its Bohr radius. The idea is then to follow concentrations of the species of interest through freeze-out to the present time, and determine the prospects for terrestrial observation of relic technibaryons; the importance of excited states is the main open question.

\section{C.4 Astrophysical Objects}

Selipsky, with B. Lynn and D. Hochron (Stanford), recently completed an analysis of the restrictions on neutron star masses, within the "Q-star" equation of state arising from soliton solutions to Walecka's hadrodynamic nuclear binding model [30]. Known nuclear physics does not rule out such self-binding-soliton equations of state for very large baryon numbers, and thus cannot rule out maximum masses of neutron stars well above the "black hole limit" of about three solar masses. However, the new work shows that, assuming the $1.558 \mathrm{~ms}$ pulsar is in the Q-star state and does not shed mass or undergo non-axisymmetric gravitational wave instability, even the Q-star equation of state cannot exceed about 5.3 solar masses.

R. Rohm and a student, Indranil Das Gupta, are considering some variants of the standard cosmic-string solutions in field theory. Several situations (e.g., superconducting cosmic strings) are already well studied where the presence of additional fields beyond the minimal set required to form a cosmic string significantly changes its structure; these additional fields may arise for instance in an extended Higgs sector or be part of the matter sector of the theory. In the case presently under consideration some of the additional scalar fields develop an expectation value in the core (where the symmetry-breaking Higgs field vanishes), favoring an asymmetric arrangement of the fields when a pair of strings approach one another. If the mechanism of string formation is suitable, it may favor the formation of pairs of 'bound' cosmic strings, which may have some novel and significant differences in their behavior from ordinary cosmic strings. Currently under investigation are the proper geometric description of this type of extended object (prosaically resembling a cosmic ribbon, very much longer than its width), the classical action principle describing its motion, and the circumstances under which such objects occur in grand-unified theories and are formed in the early universe.

\section{New Field-Theoretic Methods and Models}

Theoretical physics has enjoyed renewed vitality in recent years, due partly to the challenges presented in understanding string theory as a model of fundamental particle 
physics. There have been both new developments and new insights on old problems. Researchers at Boston University have been active in several areas.

\section{D.1 Chern-Simons Gauge Theories in $2+1$ Dimensions}

$\mathrm{Pi}$ has been working on Chern.-Simons gauge theories in three-dimensional space-time. These theories are of pedagogical interest since they may teach us lessons relevant to the physical four-dimensional world. They are also mathematically interesting, giving rise to effects and structures that invite further study. Moreover, they. may have a role in the descriptions of actual laboratory processes that are confined to a plane: the quantum Hall effect and high- $T_{c}$ superconductivity.

In the last few years, $\mathrm{Pi}$ and her collaborators have studied various aspects of $\mathrm{N}$ charged particles interacting through $\mathrm{U}(1)$ Chern-Simons gauge fields. As an approach to the general $\mathrm{N}$-body problem, $\mathrm{Pi}$ and Jackiw constructed a non-relativistic field theory model which is a second quantized description of nonrelativistic N-body quantum mechanics. On the classical level, a gauged non-linear Schrödinger equation emerges in this model. They found explicit, two-dimensional static, self-dual solutions that satisfy the Lioville equation [31]. Contrary to assertions in the literature that "the effect of ChernSimons terms is to transmute the statistics of the particles and to do nothing else" [32], these explicit solutions vividly demonstrate that the Chern-Simons terms support nonperturbative excitations whose role in quantum field theory still needs to be explored.

$\mathrm{Pi}$ and Jackiw have studied other properties of their model [33]: its explicit and hidden symmetries, its relation to a relativistic field theory [34] and its supersymmetric generalization. Moreover, they have studied some of the time-dependent solutions which are obtained from static self-dual solutions by space-time transformations. These solutions represent the self-dual solutions in the presence of external magnetic field or harmonic force. They are periodic in time; $\mathrm{Pi}$ and Jackiw were able to quantize them by the BöhrSommerfield procedure and obtained semi-classical energy quantization [35].

$\mathrm{Pi}$ and her collaborators have also studied non-Abelian nonrelativistic field theories on a classical level. They have shown that the self-dual nonrelativistic, non-Abelian systems lead to the Toda hierarchy of integrable equations which provides a unified framework for understanding the properties of these equations [36]. 
Pi's current work concerns the quantum mechanics of $\mathrm{N}$-particles interacting through non-Abelian Chern-Simons theories. When charged matter is interacting with pure ChernSimons gauge fields, the gauge fields are completely specified in terms of the matter variables by a field-current identity. In the Abelian case, the field-current identity is explicitly soluble and the gauge fields are expressed in terms of particle coordinates - they are singular pure gauge, which leads to fractional spin and statistics. A complete quantization has been carried out for the Abelian case, although the N-body solutions of the Schrödinger equation are not known except for the case of two-body problem. $\mathrm{Pi}$ is currently working on the systematic quantization of $\mathrm{N}$-body quantum mechanics interacting through nonAbelian Chern-Simons gauge fields.

\section{D.2 Two-Dimensional Models of Weak Anomalous Symmetry Violation}

Cohen and Rohm investigated whether two-dimensional models could capture some of the essential features of the 'weak-instantons' of the standard-model SU(2) gauge group, namely the presence of exponentially small breaking of global symmetries (such as baryon number) at low energies, which becomes much stronger at higher energy scales. The subject of instanton effects in the weak interactions is an old one, but not thoroughly explored until recently. In his classic paper, ' $t$ Hooft [37] showed that although there may be no instanton solutions in a spontaneously-broken gauge theory like the standard model, the symmetrybreaking effects due to nonperturbative, topologically nontrivial gauge-field configurations may still be present, although unobservably small. Because these effects were supposed to be so tiny, they were for a long time ignored, in contrast to the well-studied effects of instantons in unbroken gauge theories such as color $\mathrm{SU}(3)$. More recently this anomalous symmetry-breaking has been conjectured to lead to appreciable baryon number violation in the standard model at high temperatures [38] or in high-energy scattering [39].

The status of these effects remains somewhat controversial, due at least in part to the absence of tractable theoretical models. The question of whether semiclassical calculations of these effects are reliable can be investigated in lower-dimensional models of instantons in unbroken non-Abelian gauge theories. Progress on this question was advanced in part by the study of analogous instanton effects in the two-dimensional $\mathrm{CP}^{N-1}$ sigma model. Like

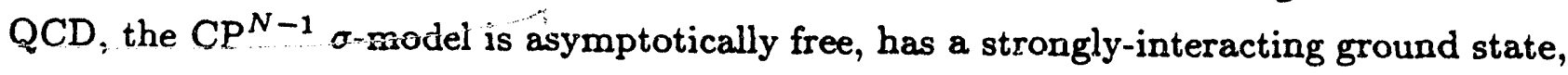
and exhibits instanton field configurations. Because the nonperturbative effects could 
also be studied $[40][41][42]$ through the $1 / N$ expansion for large $N$, these sigma models were a useful theoretical paradigm. Comparison of the large- $\mathrm{N}$ saddlepoint calculations to the semiclassical approximation helped clarify some of the outstanding questions about instanton effects.

Our investigation led us to modify the two-dimensional $C P^{N-1}$ sigma-model Lagrangian by adding a potential in the $\sigma$-model coordinates (which breaks the $S U(N)$ global symmetry to $S U(N-1)$ ), as a possible analogue of the standard-model weak instantons. Because of the potential there are no stable semiclassical solutions of finite action to the Euclidean field equations, but there are approximate solutions of finite action, which give rise to nonperturbative symmetry violation.

We formulated the $1 / \mathrm{N}$ expansion in this 'softly-broken $C P^{N-1}$ ' model through a saddlepoint evaluation of the functional integral and determined that the validity of this approximation depended on keeping the explicit symmetry-breaking at a fixed ratio to the dynamically-generated mass scale in the large $\mathrm{N}$ limit. This assures that the heavier degrees of freedom are not fully decoupled, so that an expansion in powers of $1 / \mathrm{N}$ is justified. We compared the results to those obtained through perturbation theory and the additional effects of weak (or constrained) instantons, and managed to reconcile these approaches to give a picture of the physical content of the theory, and consider its suitability as a toy model for the four-dimensional phenomena mentioned above.

Cohen and Rohm plan to continue to consider some aspects of large- $\mathrm{N}$ models for weak instantons, including some detailed computations of finite-temperature effects and some variations of the basic model. In particular, the $C P^{N-1}$ sigma-model has a natural supersymmetric extension which should provide an alternate route to understand some of the same properties.

\section{D.9 Integrable Models}

Fendley has continued his study of integrable models, in particular those with interesting condensed-matter applications and with $N=2$ supersymmetry. In collaboration with H. Saleur (USC) and Al. B. Zamolodchikov (Montpellier), he studied the $O(n)$ model in its low-temperature phase, which as $n \rightarrow 0$ describes the "dense" phase of $2 \mathrm{D}$ polymers [43]. This problem is exceptionally difficult because of the presence of level crossings, a poorly-understood phenomenon. They also showed how exact $S$-matrices of massless 
particles can be used to describe a wide variety of models [44]. This includes the flows from one minimal model to another, completing a picture which had been the subject of much research in the last several years. Fendley also showed how these $S$-matrices can be applied to give a solution of the Kondo problem (an impurity coupled to an electron gas) directly in the continuum [45]. This reproduced earlier Bethe ansatz results in a much more intuitive manner.

In collaboration with K. Intriligator (Rutgers), Fendley found $S$-matrices which give an exact solution of the Landau-Ginzburg models which flow into the $N=2$ minimal models [46]. Because of their crucial role in string theory, topological field theory, $2 \mathrm{~d}$ gravity and mirror symmetry, these models and their Landau-Ginzburg description have been intensely studied. This result shows that one can approach these models as particle theories instead of field theories, and derive exact results like a $c$-function and the elliptic genus. Recently, C. Vafa and E. Verlinde have found evidence that these flows can describe a black-hole-like object.

Fendley and Intriligator have also developed a new method of checking exact $S$ matrices for models with a $U(1)$ symmetry [47]. This gives a simple check independent of that resulting from the dilogarithms of the thermodynamic Bethe ansatz.

In addition, Fendley has given an invited talk at the SUSY ' 93 conference, which will appear in the proceedings [48]. With $\mathrm{H}$. Saleur, he also will be preparing a set of lectures to be delivered at the Trieste school.

\section{D.4QED in $2+1$ Dimensions}

Terning, with Appelquist (Yale) and Wijewardhana (Cincinnati), is working on the chiral symmetry breaking phase transition in $2+1$ dimensional QED. There has been a recent controversy over whether the transition is first or second order. Appelquist, Terning, and Wijew urdhana intend to resolve the matter by calculating the renormalized mass of the particle created by the order parameter field. If this mass goes to zero at the transition point, then the transition is second order, as suggested by lattice simulations. 


\section{D.5 Qualitons}

Selipsky has studied Kaplan's proposal that skyrmion-like "qualitons" solve the problem of connecting the successful constituent-quark phenomenological model to the fundamental degrees of freedom of QCD. Topological solitons of a color- $S U(3)_{L \times R}$ chiral Lagrangian were conjectured to model constituent quarks and their sub-confinement-range color dynamics; Karliner et al. derived the model (in $1+1$ dimensions) as an exact nonAbelian bosonization QCD. Selipsky found variational solutions to the simplest model in 3+1 dimensions, consistent with numerical solutions he also obtained working with Gomelski and Karliner (Tel Aviv). The results [49] indicate that these qualitons naturally tend to be heavier than constituent quarks, and that quantum corrections to the classical mass can be large; but some areas of Lagrangian parameter space yield more satisfactory phenomenology and controllable quantum corrections. Since color $S U(3)$ in this model replaces the flavor $S U(2)$ of the original Skyrme Lagrangian, there are several additional stabilizing four-derivative terms in the Lagrangian, each adding a free parameter to the theory. The work on $1+1$ dimensional solitons suggests that qualitative features of the results will not be affected by such generalizations. Future work should confirm this, and possibly lead to useful phenomenology. If it is possible to restrict the parameter space, the results could provide a new viewpoint on constituent quarks, and a numerical framework for investigating their behavior.

\section{E. Nonperturbative Investigations of Quantum Field Theories}

Since the introduction of quantum field theory, perturbative expansions have been a primary instrument for deriving quantitative predictions. Nevertheless, many extremely important phenomena, such as the confinement of quarks within hadrons or the instanton mediated baryon number violation, are of non-perturbative origin. In order to study such phenomena theorists have developed several non-perturbative techniques. Most notable among these techniques are those based on semiclassical expansions and those based on the lattice regularization of quantum gauge fields [50]. The formalism of lattice gauge theory allows for strong coupling expansions, mean field calculations, the numerical solution of the classical field equations and, especially, the implementation of powerful methods of computer simulation [51] [52] [53] [54]. Within the Boston University particle theory 
group, research based on non-perturbative techniques is currently pursued by faculty members Rebbi and Brower (Engineering), research associates Shen, Carreau and Vaughan. graduate students S. Harrington, S. Myint and R. Strilka. Carreau and Vaughan have independent (non-DOE) support and will leave in September when $R$. Levi, who has recently obtained his $\mathrm{Ph} . \mathrm{D}$. from MIT, is expected to join our group (also with independent support). Professor W. Kerler visited the group for two months this year and is expected to visit again in the fall. During previous years research associates S. Huang, E. Myers, J. Potvin and E. Vicari, together with several students and visitors have participated in this program of research.

In the course of the past few years major topics of investigation have been the inclusion of fermionic degrees of freedom in lattice calculations of the hadronic spectrum, the quantization of a theory on a null-plane lattice, the calculation of the surface tension in finite temperature QCD, the first principle derivation of chiral Lagrangians, multigrid methods for quark propagators and other acceleration techniques, the evolution and interaction of cosmic strings and superconducting vortices, the calculation of semiclassical solutions relevant for instanton mediated baryon number violation, properties of the Higgs effective potential ior the electroweak phase transition and matrix models for 2-d gravity and effective string theories. In this section we will outline only the most recent research results, research in progress and future projects of research. Results obtained in the other lines of investigation have already been described in the reports on current research included in previous proposals to the DOE.

\section{E.1 Thermodynamics Properties of Ouantum Chromodynamics}

Lattice simulations have shown that Quantum Chromodynamics undergoes a transition at high temperature to a Debye screened quark gluon plasma and that in quenched QCD, i.e., the model where one neglects the creation and annihilation of virtual quarkantiquark pairs, the transition is a phase transition of the first order. At a first order phase trausition two different phases can coexist and the surface tension, i.e., the excess free energy due to the presence of an interface, is an important physical quantity which in QCD determines the nucleation rate of hadrons as the quark-gluon plasma cools down past the phase transition. In most systems of practical interest the surface tension cannot be determined analytically and one must resort to computational methods. An efficient 
technique based on a numerical simulation experiment was developed by Potvin and Rebbi [55] and was applied to quenched QCD in a series of investigations carried out in collaboration with Brower, Huang, Ross and Sanielevici [56] [57] [58]. This method produced the value of approximately $6 \mathrm{MeV} / \mathrm{fm}^{2}$ for the surface tension on a lattice with $N_{t}=4$, as well as evidence that the transition has the property of "perfect wetting". At the Amsterdam Conference on Lattice Gauge Theories (Sept. 1992) a few groups presented results on the QCD surface tension obtained with different techniques. These were limited to a coarser lattice with $N_{t}=2$ and corroborated the value published for this system in [56]. Subsequently, values compatible with the result of our group have been independently obtained also for $N_{t}=4$. Continuing this line of investigation, Potvin and Rebbi, in collaboration with Gausterer and Sanielevici, have obtained results for the surface tension and universality in the 3-dimensional Ising model [59] and, working in collaboration with Huang, for curvature effects on the value of the surface tension in quenched QCD [60]. An important problem is to find out how the surface tension is affected by the inclusion of virtual quark-antiquark creation and annihilation effects, i.e., to extend the calculations beyond the quenched approximation. Rebbi and collaborators are planning to investigate this problem in the future, but this must await the development of more efficient techniques to deal with quark propagators in the simulations and the resolution of the question of whether the first order nature of the transition survives the inclusion of quark vacuum polarization effects.

\section{E.2 Chiral and Heavy Quark Physics}

Many physical phenomena are characterized by the fact that only a subset of all degrees of freedom play a relevant role in the dynamics, whereas the other degrees of freedom, typically corresponding to different scales of energy or distance, basically contribute just to a renormalization of the interactions. It is then possible in principle to integrate out the irrelevant degrees of freedom and to describe the phenomena in terms of an effective Lagrangian, involving only the relevant degrees of freedom. Examples are the interactions of light pseudoscalar mesons, which can be described by an effective Lagrangian formulated in terms of chiral fields, or the phenomenology of systems formed by heavy quarks, which can be treated by effective Lagrangians involving non-relativistic fields. The parameters of the effective Lagrangian are frequently constrained by symmetry principles, which reduce 
their number substantially. The remaining parameters can be fitted to a few experimental observations and the effective Lagrangian, thus determined, becomes a very powerful tool for the phenomenological study of the interactions. Howcrer the parameters of the effective Lagrangian are not arbitrary, but are formally determined by the same procedure of integration of irrelevant degrees of freed $\mathrm{m}$ which leads to the definition of the Lagrangian itself. Brower, Rebbi, Shen and graduate student S. Myint have been investigating the possibility of calculating the parameters of chiral Lagrangians used for pion and $K$-meson phenomenology directly from the fundamental QCD Lagrangian by lattice simulation techniques. In a preliminary analysis, extending some pioneering work by Kluberg-Stern et al. [61] and Kawamoto and Smit [62], Rebbi and Myint have implemented the reduction of the degrees of freedom to a system of chiral fields in the strong coupling limit. By working on an $F_{4}$ lattice, which has a larger group of symmetry than the hypercubical lattice considered in [61][62], one obtains a chiral lattice Lagrangian which, in a low momentum expansion, has the sy anmetry of the continuum Lagrangian all the way up to terms of order $p^{4}$. One can therefore relate the parameters obtained from the lattice derivation to the phenomenological parameters appearing in the continuum effective chiral Lagrangians [63]. In the strong coupling limit the quarks are too tightly bound, and this is reflected by the fact that the coefficients produced by the lattice derivation are much larger than the phenomenological values. However, the construction opens the way for a similar calculation of the coefficients of continuum chiral Lagrangians by simulation methods in the intermediate coupling domain, where a large body of lattice results indicates that one recovers the features of continuum $\mathrm{QCD}$ to a very good approximation. The plan is to use a renormalization calculation to derive the coefficients of a lattice chiral Lagrangian directly from the fundamental QCD Lagrangian. By performing a low momentum expansion one can then obtain the coefficients of the phenomenological continuum chiral Lagrangians. Work in this direction is in progress.

Another approach to the chiral properties of QCD is being explored by Brower, Rebbi and Shen, in collaboration with C. I. Tan (Brown U.) and graduate student K. Orginos (Brown U.). They are investigating the possibility that an "extended" lattice QCD Lagrangian in the spirit of the Georgi-Manohar model, which includes explicit pion fields in addition to quarks and gluons, may belong to the same universality class as the original QCD Lagrangian. A preliminary investigation by Brower, Shen and Tan for a toy model 
system with the gluonic effects replaced by a four-Fermi interaction has demonstrated universality as the cut-off is sent to infinity. This mechanism is being explored further in other toy model systems by both explicit theoretical calculations and by numerical simulations, together with Orginos. The preliminary results will be reported soon [64].

The phenomenological effect of the explicit chiral pion degrees of freedom is to increase the small current algebra mass of the quarks to their constituent mass value, which in turn should speed up dynamical fermion simulations. The numerical implementation of this idea is in progress. Regardless of the technical questions of universality for "chirally extended QCD," we believe that this hybrid model will have important phenomenological applications to the study of the chiral condensate similar to the use of chiral Lagrangians, but with the advantage that here both the point-like effects of quarks and confinement of valence quarks are being modeled in conjunction with the chiral condensate. In hot nuclear matter such a hybrid description may be very useful. In addition the basic underlying issue is the ultimate distinction between elementary and composite Higgs fields in a quantum field theory, which is of crucial importance to building theories (such as technicolor) for physics beyond the energy scale addressed by the standard model.

In the limit of heavy quark mass, the QCD action can be reduced to an effective Lagrangian which possesses additional symmetries. In such heavy quark effective theory, mariy heavy meson decay amplitudes (for example: $B \rightarrow D$ decay) can be reduced to a simple form and expressed in terms of a single unknown nonperturbative function-the Isgur-Wise function. In recent work of Shen, in collaboration with Bernard (Washington University at St. Louis) and Soni (Brookhaven), the Isgur-Wise function was calculated on the lattice. The preliminary result was presented by Shen at the Lattice ' 92 conference in Amsterdam [65] and DPF '92 at Fermi Lab [66], and the final result is presented in a more recent preprint [67]. The Isgur-Wise function is important for understanding the $B$ meson decay processes and for a model-independent determination of $V_{c b}$ in the CKM matrix of the Standard Model. The lattice result turned out to be clearly competitive with continuum model calculations and compares favorably with the ARGUS experimental data. Together with Bernard and Soni, Shen plans to investigate other processes that are important for $B$ meson physics. 


\section{E. 9 Vortices in the Abelian-Higgs Model}

The lattice regularization offers also a powerful formalism for implementing the numerical solution of classical field equations. This has been exploited at Boston University in a series of studies, conducted by Rebbi, Myers and collaborators, of the evolution and interaction of vortices in the Abelian-Higgs model, a model which can be used to describe cosmic strings as well as superconducting vortices [68] [69] [70]. Working under the guidance of Rebbi, and with additional advice from S.-Y. Pi, R. Strilka has extended these investigations to the vortex-like solutions of Chern-Simons theories, a class of particle models for particle interactions with interesting topological properties, showing that Chern-Simon vortices interact non-trivially and therefore are not solitons [71]. Strilka, who also participated in other investigations of cosmic strings and vortices [70] [71] [72], received his $\mathrm{Ph} . \mathrm{D}$. this spring. Another interesting application of the classical solutions of the Abelian-Higgs model is found in the analysis of instanton-induced baryon decay. It is well known that in theories, such as the electroweak theory, which exhibit an anomaly in the divergence of the baryonic current instanton processes, this anomaly can give origin to non-conservation of baryon number. At low energy such processes have an exceedingly small amplitude, because of a barrier which generates an exponential suppression factor, but both high temperature fluctuations and high energy collisions can overcome the effect of the barrier and thus give origin to an appreciable rate of baryon number violating transitions. While it has been established that this is indeed the case for high temperature fluctuations, the matter of whether high energy collisions can also accelerate the rate of baryon non-conservation remains very much under dispute. Recently Rubakov and collaborators [73] have suggested a framework to resolve this problem, which involves the solution of the classical equations of motion for systems with instanton transitions along a complexified space-time path. The two-dimensional Abelian-Higgs system is one of the simplest, yet realistic models where these ideas can be applied. Nevertheless, even in this case the highly non-linear equations that need to be solved can only be studied computationally. Rebbi and Vaughan have extended the formalism used for the analysis of vortex interactions to the solution of the equations of motion for complex fields and complexified paths in space-time, as required for an implementation of the semiclassical approach to baryon number violating processes. Solutions with complexified fields along the imaginary 
time axis have been obtained, and the method must now be extended to encompass a further evolution along the real time axis with some highly non-trivial boundary conditions. Work on this problem is in progress.

\section{E.4 Baryogenesis and Electroweak Transitions}

Brower and $\mathrm{Pi}$, in collaboration with $\mathrm{Pi}$ 's student Giovanni Amelino-Camelia, are extending the diagrammatic approach to finite temperature effective potentials calculations beyond the purely analytical methods used to date. Reliable and accurate methods for computing the effective potential are crucial for the description of the temperature induced phase transitions of quantum field theories in baryogenesis and hadronization. Here one begins by considering an approximate effective potential to leading (and later, possibly, subleading) daisy-superdaisy order, including the effects of the momentum dependence of the effective masses [74]. Then in order to solve the resultant integral (or gap) equation one must iterate to convergence where the inner loop is itself a numerical evaluation of Feynman integrals. This is a non-trivial computation which will be pursued both on Boston University's and MIT's Connection Machines. First, the lambda $\phi^{4}$ theory, which is interesting both for its mathematical properties and as as a toy model for the Standard Model, will be considered to develop and test the numerical procedures. Next, the methods will be extended to the analysis of the electroweak effective potential with particular attention to the question of the strength of the first order phase transition.

Shen has studied a $U(N) \times U(N)$ scalar model in four and three dimensions. Based on perturbative argument, such a model will have fluctuation-induced first order phase transitions - the Coleman-Weinberg phenomenon. In four dimensions this $U(N) \times U(N)$ symmetric model can be considered as the low energy effective action for strong extended technicolor or top crndensate models. It was suggested by Chivukula, Golden and Simmons [1] that the parameter region in which the phase transition becomes strongly first order should be excluded from the continuum theory, thus a bound might be placed on the strong coupling extended technicolor model or top condensate model. The work of Shen in four dimensions is reported in [75]. By comparing the numerical simulation results with perturbation predictions in the weak coupling region, the Coleman-Weinberg phenomenon is demonstrated explicitly. First order phase transitions were also found in the strong coupling region. In three dimensions, the $U(N) \times U(N)$ scalar model can be considered 
as a low energy effective model for the QCD at finite temperature. Thus the order of phase transition will illuminate the nature of the QCD finite temperature chiral symmetry restoration transition. A series of scenarios was suggested in the perturbative framework in [76]. In numerical investigations, Shen found clear signals of first order phase traisitions in the three dimensional model. Shen plans to continue this research further to demonstrate the renormalization group ( $R G$ ) behavior of the model in three dimensions and compare directly with the predictions obtained in the $\epsilon$-expansion.

Shen also plans to pursue a nonperturbative calculation of the effective potential for the $U(N) \times U(N)$ scalar model, working with graduate student V. Koulovassilopoulos. The idea is to use a nonperturbative iterative RG equation to derive the effective potential. Using this method one can obtain the effective potential in arbitrary dimension and derive a comprehensive picture about the order of the phase transitions as the dimension is changed. Although the method requires truncations of the action, it will provide a nonperturbative investigation of the fixed point structure in the coupling parameter space.

The order of the electroweak symmetry restoration phase transition at high temperature is an important question for cosmology. The long history of many perturbative studies has not been conclusive. Brower, Rebbi and Shen, together with graduate student Harrington, have recently developed a program on the CM-5 at Boston University to investigate this problem numerically. Although this will be a numerically very challenging problem, with the availability of the powerful computational resource of Boston University's CM-5, they expect to obtain results that will be useful.

Shen has studied the relation of the two dimensional Higgs-Yukawa model and the Gross-Neveu model in [77]. He was able to show analytically that both models have similar phase diagrams and possess the property of asymptotic freedom. This work is complementary to a numerical investigation in [78] and suggests that the two dimensional Higgs-Yukawa model is equivalent to the Gross-Neveu model.

A research project on the dynamics of the phase transition of the $U(1)$ lattice gauge theory was started during a visit of Werner Kerler of the University of Marburg, who spent two months this year with our group. Although the $U(1)$ gauge theory is non-interacting in the continuum, where it describes the free photon field, it acquires a non-trivial, interacting structure on the lattice because of the non-linearities induced by the discretization. In particular, the lattice $U(1)$ model undergoes a phase transition whose properties have 
been at the center of many investigations for the implications that they may carry for particle models. In previous research Rebbi has studied the role that magnetic monopoles play in the dynamics of the $U(1)$ phase transition [79]. Kerler has recently developed a computational method that allows one to explore the behavior of a phase transition in an extended space of coupling parameters. In an ongoing investigation, Ferler and Rebbi are applying this novel technique to a study of the $U(1)$ phase transition in an extended space of couplings that include a monopole chemical potential, with the expectation that this will help clarify the properties of this interesting system.

\section{E.5 Related Professional Activities}

In related activities Brower and Rebbi, following a longstanding interest in the improvement of computational techniques for lattice calculations, have formulated a project for "Software and Algorithm Development for Lattice Quantum Chromodynamics" involving the participation of fifteen scientists from twelve U.S. institutions. This was submitted as a formal proposal to the DOE High Performance Computing and Communications program. Unfortunately the program itself was not funded this year, so that no proposal could be considered for support, but Brower and Rebbi plan to pursue further the organization of collaborative algorithm development efforts, which they deem crucial for the progress of lattice calculations and valuable to the whole community of researchers.

Brower, Rebbi and Shen are members of the QCD Teraflop collaboration, for which Brower also serves as member of the steering committee. Brower, in collaboration with computer architects at MIT's Lincoln Laboratory and Thinking Machine Corporation, is now engaged in a careful review of the architectural constraints of lattice QCD (and a few closely related field theoretical or many-body quantum applications). The purpose is to establish the feasibility of a cost-effective design based on the next generation of chip technology, which will deliver a sustained teraflop performance on this application space. This design study will issue its findings to the Teraflop Collaboration in the fall of 1993 and soon thereafter will form part of the technical basis for a proposal to fund the teraflop project. As part of this project Brower is also engaged in collaboration with John Negele and several graduate students in writing a special assembly language library for QCD to optimize performance of our code on the CM-5. 
Rebbi and Brower continue to play an important role in the guidance of the Boston University Center for Computational Science, which, with its Connection Machine and other facilities, provides support to a wide range of computationally based research, including a large part of the investigations outlined in this section. Rebbi is the Center's director and Brower is a member of its scientific council.

Together with John Negele of MIT, Rebbi has been the principal organizer of a research seminar on lattice gauge theories which has met weekly, alternating between $\mathrm{BU}$ and MIT, throughout the academic year. Students, research associates and faculty alike all greatly benefited from the presentations and research discussions which were held during the seminar. 


\section{References}

[1] R. S. Chivukula, E. H. Simmons, and M. Golden, "Critical Constraints on Chiral Hierarchies," Phys. Rev. Lett. 70, 1587 (1993).

[2] "Non-Oblique Effects in the $Z b \vec{b}$ Vertex from ETC Dynamics," (with S. B. Selipsky and E. H. Simmons), Phys. Rev. Lett. 69, 575 (1992).

[3] "Walking Technicolor and the $Z b \bar{b}$ Vertex," (with E. Gates, E. H. Simmons, and J. Terning), Boston University Preprint BUHEP-93-11, and Phys. Lett. B, in press.

[4] D. Kominis and R. S. Chivukula, "Triviality Bounds in Two-Doublet Models," Phys. Lett. B 304, 152 (1993).

[5] R. S. Chivukula and V. Koulovassilopoulos, "Phenomenology of a Non-Standard Higgs," Boston University Preprint BUHEP-93-7, and Phys. Lett. B, in press.

[6] R. S. Chivukula, M. Golden, and M. Dugan, "Analyticity, Crossing Symmetry and the Limits of Chiral Perturbation Theory," Phys. Rev. D47, 2930 (1993).

[7] R. S. Chivukula, M. Golden, and M. Dugan, "Electroweak Corrections in Technicolor Reconsidered," Phys. Lett. B 292, 435 (1992).

[8] A. Cohen, G. Ecker and A. Pich, "Unitarity and $K_{L} \rightarrow \pi \gamma \gamma$," BUHEP-93-5; to appear in Physics Letters.

[9] NA31 Collab., G. D. Barr et al., Phys. Lett. B284, 440 (1992).

[10] E73i Collab., V. Papadimitriou et al., Phys. Rev. D44, 573 (1991).

[11] GEM Technical Design Report, GEM-TN-93-262, submitted to the Superconducting Super Collider Laboratory, April 30, 1993.

[12] "Hiding the Electroweak Symmetry Breaking Sector" (with M. Golden), Phys. Lett. B 267, 233 (1991).

[13] "Phenomenology of a Hidden Symmetry Breaking Sector" (with M. Golden D. Kominis and M. V. Ramana), Phys. Lett. B 293, 400 (1992).

[14] "Hiding the Electroweak Symmetry Breaking Sector" (with M. Golden, D. Kominis, and M. V. Ramana), Proceedings of High Energy Physics with Colliding Beams (Yale University, New Haven, Oct. 2-3, 1992); Boston University preprint BUHEP-92-34.

[15] K. Lane and M. V. Ramana, Phys. Rev. D44, 2678 (1991).

[16] L. Dolan and R. Jackiw, Phys. Rev. D9, 3320 (1974);

S. Weinberg, Phys. Rev. D9, 3357 (1974);

D. Kirzhnitz and A. Linde, JETP Lett. 40, 628 (1974).

[17] J. M. Cornwall, R. Jackiw and E. Tomboulis, Phys. Rev. D10, 2428 (1974).

[18] G. Amelino-Camelia and S.-Y. Pi, Phys. Rev. Lett. 47, 2356 (1993).

[19] S.-Y. Pi, unpublished.

[20] G. Amelino-Camelia, submitted to Phys. Rev. D.

[21] A. Sakharov, Zh. Eksp. Teor. Piz. Pis'ma 5, 32 (1967). 
[22] S. Dimopoulos and L. Susskind. Phys. Rev. D18, 4500 (1978); M. Yoshimura. Phys. Rev. Lett. 41, 281 (1978); (E) 42, 746 (1978); D. Toussaint, S. Treiman, F. Wilczek and A. Zee, Phys. Rev. D19, 1036 (1979).

[23] A. Cohen, D. Kaplan and A. Nelson, Phys. Lett. B245, 561 (1990).

[24] A. Cohen, D. Kaplan and A. Nelson, Nucl. Phys. B373, 453 (1992).

[25] A. Cohen and A. Nelson, Phys. Lett. B297, 111 (1992).

[26] "Progress in Electroweak Baryogenesis," A. Cohen, D. Kaplan, and A. Nelson, BUHEP-93-4, to appear in Annual Review of Nuclear and Particle Science.

[27] R. S. Chivukula, A. Cohen, M. Luke, and M. Savage, "A Comment on the Strong Interactions of Color-Neutral Technibaryons," Phys. Lett. B 298, 380 (1993).

[28] S. Nussinov, Phys. Lett. B279, 111 (1992).

[29] R. S. Chivukula and T. P. Walker, Nucl. Phys. B329, 445 (1990).

[30] "An Upper Bound on Q-star Masses," D. A. Hochron, B. W. Lynn, and S. B. Selipsky, SU-ITP-92-09; Class. Quant. Grav. 10, 299 (1993).

[31] R. Jackiw and S.-Y. Pi, Phys. Rev. Lett. 64, 2969 (1990).

[32] See, for example, F. Wilczek, "Lectures at the Ferrava School," preprint IAS - SNS HEP - 89/50 (1989).

[33] R. Jackiw and S.-Y. Pi, Phys. Rev. Lett. 64, 2989 (1990).

[34] R. Jackiw and S.-Y. Pi, Phys. Rev.D42, 3500 (1990);

R. Jackiw, S.-Y. Pi and E. Weinberg, p. 268 in proceedings of Progress in High Energy Physics, W. Pauchy Hwang, S.-C. Lee, C.-E. Lee and D. Ernst, eds. (Amsterdam: North Holland, 1991).

[35] R. Jackiw and S.-Y. Pi, Phys. Rev. Lett. 67, 415 (1991);

Z. Ezawa, M. Hota and A. Iwazaki, Phys. Rev. Lett. 67, 411 (1991);

R. Jackiw and S.-Y. Pi, Phys. Rev. D44, 2524 (1991);

R. Jackiw and S.-Y. Pi, Yukawa International Seminar, Kyoto, Japan, July/August 1991.

[36] G. Dunne, R. Jackiw, S.-Y. Pi and C. Trugenberger, Phys. Rev. D43, 1332 (1991).

[37] G. 't Hooft, "Symmetry Breaking Through Bell-Jackiw Anomalies," Phys. Rev. Lett. 37, 8 (1976).

[38] V.A. Kuzmin, V.A. Rubakov and M.E. Shaposhnikov, "On the Anomalous Eillectroweak Baryon Number Nonconservation in the Early Universe," 155B, 36 (1985).

[39] Ringwold, etc.

[40] A. D'Adda, M. Luscher and P. Di Vecchia, Nucl. Phys. B146, 63 (1978).

[41] E. Witten, Nucl. Phys. B149, 285 (1979).

[42] I. Affleck, "Testing the Instanton Method," Phys. Lett. 92B, 149 (1980); "The Role of Instantons in Scale Invariant Gauge Theories," Nucl. Phys. B162, 461 (1980); "The 
Role of Instantons in Scale Invariant Gauge Theories 2: The Short Distance Limit." Nucl. Phys. B171, 420 (1980).

[43] P. Fendley, H. Saleur and Al. B. Zamolodchikov, hepth@xxx/9304050, "Massless Flows

I: The Sine-Gordon and $O(n)$ Models," BUHEP-93-8, USC/93-003, LPM-93-07, to

[44] P. Fendley, H. Saleur and Al. B. Zamolodchikov, "Massless Flows II: The Exact $S$ Matrix Approach," hepth@xxx/9304051, BUHEP-93-9, USC/93-004, LPM-93-08, to appear in Int. J. Mod. Phys. A.

[45] P. Fendley, "Kinks in the Kondo Problem" (cond-mat@babbage.sissa.it/ 9304031),

[46] P. Fendley and K. Intriligator, "Exact $N=2$ Landau-Ginzburg Flows," BUHEP-93-17.

[47] P. Fendley and K. Intriligator, "Central Charges without Finite-Size Effects," hepth@xxx/9307101, BUHEP-93-16, RU-93-26.

[48] P. Fendley, "Exact Information in $N=2$ Theories," hepth@xxx/9305124, BUHEP-9313.

[49] "Static Properties of Quark Solitons," G. Gomelski, M. Karliner, and S.B. Selipsky, BUHEP-92-17, hep-ph@xxx.lanl.gov/9304217.

[50] K. Wilson, Phys. Rev. D10, 2445 (1974).

[51] M. Creutz, L. Jacobs and C. Rebbi, Phys. Rev. Lett. 42, 1390 (1979).

[52] M. Creutz, L. Jacobs and C. Rebbi, Phys. Rep. 95, 201 (1983).

[53] Lattice Gauge Theories and Monte Carlo Simulations, C. Rebbi ed., World Scientific Publishing Co., Singapore (1983).

[54] R. C. Brower, Les Houches Summer School Lectures, Theories de Jauge en Physique des Hautes Energies, North-Holland Publishing Co. (1983).

[55] J. Potvin and C. Rebbi, Phys. Rev. Lett. 62, 3062 (1989).

[56] S. Huang, J. Potvin, C. Rebbi and S. Sanielevici, Phys. Rev. D42, 2864 (1990).

[57] R. Brower, S. Huang, J. Potvin and C. Rebbi, Phys. Rev. D46, 2703 (1992).

[58] R. Brower, S. Huang, J. Potvin, C. Rebbi and J. Ross, Phys. Rev. D42, 4736 (1993).

[59] H. Gausterer, J. Potvin, C. Rebbi and S. Sanielevici, Physica A192, 525 (1993).

[60] S. Huang, J. Potvin and C. Rebbi, Int. Journal of Mod. Phys. C, to be published (1993).

[61] H. Kluberg-Stern, A. Morel, O. Napoly and B. Petersson, Nucl. Phys. B190, 504

[62] N. Kawamoto and J. Smit, Nucl. Phys. B192, 100 (1981).

[63] S. Myint and C. Rebbi, preprint in preparation and talk to be given at the International Conference on Lattice Gauge Theories (SSC Lab, Oct. 1993).

[64] R. Brower, Y. Shen and C. I. Tan, "Chirally Extended QCD," preprint in preparation.

[65] C. Bernard, Y. Shen and A. Soni, Nucl. Phys. B30 (Proc. Suppl.), 473 (1993). 
[66] C. Bernard, Y. Shen and A. Soni, DPF '92 Proceedings, p. 541.

[67] C. Bernard, Y. Shen and A. Soni, preprint BUHEP-93-15.

[68] K. Moriarty, E. Myers and C. Rebbi, Phys. Lett. B207, 411 (1988).

[69] K. Moriarty, E. Myers and C. Rebbi, Comp. Phys. Commun. 54, 273 (1989).

[70] E. Myers, C. Rebbi and R. Strilka, Phys. Rev. D45, 1355 (1992).

[71] R. Strilka, submitted to Phys. Rev. D.

[72] R. Strilka, Comp. Phys. Commun., to be publisined (1993).

[73] V. Rubakov and P. Tinyakov, Phys. Lett. B279, 165 (1992); V. Rubakov, D. Son and P. Tinyakov, Phys. Lett. B287, 342 (1992).

[74] G. Amelino-Camelia and S-Y. Pi, Phys. Rev. D47, 2356 (1993).

[75] Y. Shen, preprint BUHEP-93-5.

[76] R. D. Pisarski and F. Wilczek, Phys. Rev. D29, 338 (1984).

[77] Y. Shen, Phys. Lett. B304, 295 (1993).

[78] A. K. De, E. Focht, W. Franzki and J. Jersák, Phys. B (Proc. Suppl.) 30, 662 (1993).

[79] V. Grösch, K. Jansen, J. Jersak, C. Lang, T. Neuhaus and C. Rebbi, Phys. Lett. B162, 171 (1985). 
TASK F - MUON G-2 EXPERIMENT AT BNL

$\begin{array}{ll}\text { Faculty: } & \begin{array}{l}\text { Professor F. Krienen (ADP) } \\ \text { Professor J.P. Miller (NSF) } \\ \text { Professor B.L. Roberts (NSF) } \\ \text { Professor L.R. Sulak } \\ \text { Assistant Professor W. Worstell (P.I.) }\end{array} \\ & \text { R. Carey } \\ \text { Research Faculty: } & \text { D. Brown } \\ \text { Graduate Students: } & \text { Brookhaven National Laboratory, } \\ \text { Collaborating Institutions: } & \text { Fairfield, Heidelberg, Illinois, } \\ & \text { Institute for Nuclear Physics (Novosibirsk), } \\ & \text { Los Alamos, KEK, Tokyo, Yale }\end{array}$

\section{Project Overview:}

A new precision measurement of the muon anomalous magnetic moment is being prepared at Brookhaven National Laboratory (E821). We plan to improve the precision of this measurement by a factor of 20 over that of previous CERN experiments; this will allow measurement of the contribution from electroweak radiative corrections for the first time. In addition to contributions from virtual $W$ and $Z$ bosons, a high-precision measurement of the muon anomaly is sensitive to standard model contributions from QED (vacuum polarization) and QCD (hadronic vacuum polarization). A muon g-2 measurement consistent with standard model predictions at the level of sensitivity of E821 (0.35 ppm) will put limits on muon substructure up to $\mathrm{TeV}$ energy scales, and will put significant constraints on $\mathrm{W}$ boson substructure and a $\mathrm{W}$ anomalous magnetic moment. The E821 experiment will also provide the most sensitive search to date for a muon electric dipole moment, which would violate $T$ and $P$ invariance if nonzero.

Construction of the precision storage ring for E821 is well under way, with data-taking expected to commence in late 1995. The muon anomaly will be determined by measuring the rate of precession of muon spins relative to muon momenta in a precisely measured magnetic field. Since electrons with highest energy in the lab frame are preferentially 
emitted when the muon spin is pointing opposite its momentum vector, the spin precession frequency modulates the distribution of arrival times for high-energy decay electrons. Decay electrons spiral inward from the muon storage region until they strike one of 24 calorimeters which measure their energy and arrival times. The $3.0 \mathrm{GeV} / \mathrm{c}$ momentum of the stored muons is chosen so that electrostatic focusing fields will not affect the precession frequency of muons centered within the storage region (through a cancellation of terms). The 1.5 Tesla field in the storage region will be provided by a monolithic superferric " $C$ " magnet with a 7-meter radius, and will be monitored by fixed and movable NMR probes; the field integral over azimuth will be uniform to within $1 \mathrm{ppm}$ after shimming, and will be measured to within $0.1 \mathrm{ppm}$. The 4 superconducting coils which will drive the 700 -ton iron yoke have been wound, and construction of the yoke began in spring 1993.

In 1993, the Boston University DoE-supported g-2 group has focused its efforts on two aspects of E821:

1) the JMD-2 collaboration with Russian physicists in measuring the standard-model contribution to the muon anomaly from hadronic vacuum polarization, and

2) the design, construction, and testing of front-end data acquisition and photomultiplierassociated electronics for E821.

3) detailed Monte Carlo simulation of integrated system responses (storage ring, detector, and electronics) to a variety of running conditions, with the goal of constraining future systematic errors within acceptable limits.

Progress in each of these efforts will be outlined below, followed by our plans for 1994 .

\section{Project Progress Report and Status Summary}

\section{CMD-2 at Novosibirsk}

The QED contributions to the muon anomalous magnetic moment are well understood, as evidenced by the prenise agreement between theory and exn-riment in the measurement of the electron anomalous magnetic moment ( $\pm 45 \mathrm{ppb}$ ). Missive virtual particles contribute to the muon anomaly much more than to the electron anomaly, in proportion to the muon/electron mass ratio squared. Consequently, while the electron anomalous magnetic 
moment measurements are sensitive only to QED contributions, measurements of the muon anomaly are sensitive to the production of virtual hadrons. The most recent CERN measurements of the muon anomaly, at the $7 \mathrm{ppm}$ level, were the first to sense the contribution from hadronic vacuum polarization; the total hadronic vacuum polarization contribution is about $60 \mathrm{ppm}$. Since it involves primarily low-energy hadronic processes, the precise magnitude of the hadronic vacuum polarization contribution cannot be calculated from first principles. It must instead be inferred from measurements of $e^{+} e^{-}$hadroproduction total cross sections. Through dispersion theory, the production of real hadrons in $e^{+} \epsilon^{-}$ collisions may be related to the production of virtual hadrons in hadronic vacuum polarization. The principal uncertainty in the hadronic vacuum polarization contribution arises from uncertainty in $e^{+} e^{-}$hadronic total cross- sections. We are actively collaborating in the CMD-2 experiment at the Budker Institute of Nuclear Physics in Novosibirsk, Russia, to reduce these uncertainties below their current $\pm 1.5 \mathrm{ppm}$ level.

During its April-July 1993 running period, the Cryogenic Magnetic Detector (CMD-2) at the VEPP-2M colliding beam facility at the Budker Institute Nuclear Physics (BINP) in Novosibirsk, $P$ :esia, collected more than one inverse picobarn of data on the $\phi$ meson resonance (figure 1). This data set adds to 25 inverse nanobarns collected on the $\rho$ and $\omega$ resonances in spring 1992, and 180 inverse nanobarns collected on the $\phi$ in June 1992. During August-September 1993 a new BGO end-cap calorimeter will be installed, and starting in October 1993 several inverse picobarns of data will be collected on the $\rho$ and $\omega$ resonances. The principal physics goal of this upcoming run will be to measure total hadronic cross-sections in the energy range which is most important for muon $\mathrm{g}-2$ hadronic vacuum polarization ( $<1 \mathrm{GeV} \mathrm{CM}$ energy). Systematic and statistical errors limit our knowledge of the hadronic vacuum polarization contribution to the muon $\mathrm{g}-2$ to about 1.5 ppm; with the new Novosibirsk data we should be able to reduce this uncertainty to well under $1 \mathrm{ppm}$.

Boston University contributions to CMD-2 have taken the form of manpower, equipment, and double-checking of Novosibirsk analyses. Boston University graduate student Doug Brown has been in residence at the BINP in Novosibirsk since September 1992; he plans to work in Boston during the summer 1993 CMD-2 shutdown and then to return to Novosibirsk for the fall CMD-2 run. Asst. Prof. Bill Worstell visited the BINP in December 1992 and assisted in preparations for the spring 1993 run. Dr. Boris Khazin 
and Dr. Genia Solodov of the BINP visited Boston University for two weeks each during 1993, and Edward Anashkin from BINP will be working as a graduate research assistant with our group during the FY94 academic year. In addition, several Boston University undergraduate research assistants have been working with the CMD-2 data, with one student (Steve Doulas) completing a senior thesis on "Particle Identification through $\mathrm{dE} / \mathrm{dX}$ Measurements with the CMD-2 Drift Chamber."

While our principal interest in CMD-2 is the determination of the muon $\mathrm{g}-2$ hadronic vacuum polarization contribution through measurements of total cross-sections (including precise determinations of integrated luminosity), we have also engaged in ancillary studies of particle identification, drift chamber calibrations, and schedule optimization for the $\omega$ and $\phi$ scans. In addition to contributing data acquisition and analysis computer resources to CMD-2, we have contributed two scintillating fiber hodoscopes as part of the CMD2 luminosity monitoring system. These hodoscopes were designed and built at Boston University, incorporating $2 \mathrm{~mm}$ diameter radiation-hard scintillating fibers and 64-channel multianode photomultipliers. NIM and CAMAC modules for the readout of these devices were also designed and built at Boston University specifically for the CMD-2 luminosity monitor.

Both fiber hodoscopes are now installed at CMD-2, where they operate in coordination with a BGO crystal in the detection of single- and double-photon bremsstrahlung ("beamstrahlung") in $e^{+} e^{-}$collisions. By using a thin converter between the interaction point and the fiber hodoscope (before the BGO), one can determine the beam spot position for photons striking the BGO and correct for systematic variations in the BGO crystal's response. This beam-spot measurement is also very useful for the VEPP-2M machine operator:s, and a real-time 3-D plot of the beam spot position has been provided on a video monitor to the machine operators for their use during beam tuning (figure 2).

\section{Muon g-2 Front-End Electronics}

Boston University has assumed lead responsibility in the design, construction, and testing of PMT bases, wave-form digitizing (WFD) ADC electronics, high-speed multihit TDC electronics (MTDC), and high-speed clock distribution electronics for the $\mathrm{g}-2$ experiment. The design of a rapidly gated high-rate PMT base is closely coupled to the choice of PMT for g-2 detector readout; specifications for both devices and our progress in 
meeting these specs will be discussed below. In the design of the WFD, MTDC, and clock distribution systems we have taken advantage of development work for other experiments which has been carried out at the BU Electronics Design Facility (EDF); the MTDC is based upon an $800 \mathrm{MHz}$ GaAs gate array which was developed for the DUMAND experiment, while the WFD is very similar to the waveform digitizer developed for the MACRO experiment. The clock distribution is similarly modeled after that developed for MACRO, at least in its initial implementation. With the recent successful production of MACRO WFD boards and DUMAND MTDC gate arrays, we will now begin precision testing of systematic variations in the response of each device (e.g., rate-dependent timing shifts). We will then iterate the design of each device until we are able to meet the very demanding g-2 specifications.

The g-2 experiment involves the measurement of the arrival times of electrons with energies above a given threshold. These precise timing measurements must be made a few tens of microseconds after the "flash" of scintillation light generated by electromagnetic debris striking calorimeters at injection time. The extremely small systematic errors which we require imply that rate-dependent timing shifts (from pile-up and other effects) must be constrained to be less than 60 picoseconds on average over a 1 millisecond observation time. These requirements determine the specifications of the PMTs and their bases, and of the data acquisition electronics and calibration system.

The g-2 experiment will use 1002 " PMTs to read out calorimeter segments and 100 1 " or 2" PMTs to read out scintillator timing strips. For timing we require less than 60 ps systematic shifts between $5 \mathrm{MHz}$ and $\mathrm{DC}$ operation, and for energy measurements we require less than $0.5 \%$ systematic gain shifts over the same range of input rates. For both applications, PMTs must be gated off during the initial "flash" of charged particles accompanying injection into the storage ring; the PMT must recover after gating to operate within the above specs in less than 20 microseconds. Substantial recovery of the PMT within a few microseconds after gating is desirable, as are narrow pulse widths to minimize pile-up.

After considerable design and testing efforts, we have produced a prototype high-rate gated base which allows a Hamamatsu R1828 PMT to meet all the above specifications. Fast gating was achieved by switching a focusing electrode with a high-voltage pulse, 
achieving an extinction coefficient of $10^{5}$ and recovering from gating in less than $10 \mathrm{mi}$ croseconds (figure 3). High-rate operation was achieved with a fully transistorized dynode chain, with demonstrated timing shifts of less than 20 ps between $5 \mathrm{MHz}$ and DC for $100 \mathrm{mV}$ pulses. The test system which was used to demonstrate this PMT/base performance is a prototype for the E821 calibration system.

In recent months, considerable progress has been made on the waveform digitizer (WFD) which we are developing in collaboration with the BU MACRO group. A new design was completed in spring 1993, and 5 boards have been fabricated and stuffed. Efficient and competent vendors have been found for both tasks. Most of the features of the board have been tested successfully. The major exception is DMA readout, which did not work reliably in the last version of the board, but is not critical to the MACRO group and must be perfected by $\mathrm{g}-2$. The MACRO group is currently developing a clock distribution system for the WFDs which will serve as a prototype for the g-2 clock distribution; the latter has considerably more stringent requirements for stability and fidelity, and must be coupled to the LORAN-based g-2 system clock.

The g-2 group is beginning to test the new WFD modules. One was used as an auxiliary readout device for the calorimeters in a recent beam test at Brookhaven. Although the WFD is intended to run freely, it can be operated much as a conventional ADC, with START and STOP pulses instead of a gate. We read out the calorimeter in two different modes: sampling the summed calorimeter signal every $5 \mathrm{ns,}$ and sampling every $2.5 \mathrm{~ns}$ by using two WFD channels for a single calorimeter (figure 4). Comparing the energy spectrum produced in the calorimeter by $4 \mathrm{GeV}$ electrons, sampling the signal with the WFD (biphase mode) and with a conventional charge integrating ADC, we find an average difference of only 3 percent. This comes as no surprise, as the PMT signals were fairly wide. The performance of the WFD on faster signals remains to be tested. The other principal unanswered question is whether or not the pulse height response of the WFD remains stable with changes in the signal rate. We plan to address this question in the next few weeks, with a setup much like that used for testing our PMTs.

WFD software, developed by the MACRO group under OS-9, has been ported with little difficulty to VxWorks. In the next few weeks, we will install UNIDAQ at $B U$. UNIDAQ is a data acquisition system which has been developed at the SSC and which runs under VxWorks. UNIDAQ is a prime candidate for the $\mathrm{g}-2$ data acquisition system, and it 
will provide us with a sophisticated and uniform environment for controlling, reading out. and monitoring the performance of the WFD. We anticipate that g-2 specific tests will be completed by the end of 1993 .

The MTDC 1 ns-resolution multi-hit timing system is patterned after the multi-hit TDC developed for the DUMAND experiment. In particular, it incorporates the same direct-coupled FET logic GaAs gate array - the Vitesse VGFX200K. This applicationspecific integrated circuit (ASIC), with an area of $1.4 \times 0.8 \mathrm{~cm}$, has perhaps the largest speed*area product of any device currently in production. After some initial fabrication difficulties, all recent ASICs have performed according to specifications once cooled to 20 degrees $\mathrm{C}$ case temperature, and ASICs from the most recent production run have performed properly at room temperature. A CAMAC-based test interface has been constructed at BU and is currently being debugged; the design of the final VME-based MTDC system will draw upon our experience with this test system. In particular, we will perform rate-dependence tests on the prototype MTDC, both in stand-alone mode and in conjunction with an upstream de-randomizing circuit which was developed and built at Brookhaven National Laboratory.

\section{Monte Carlo Simulations}

G2GEANT, our GEANT-based detector simulation, has undergone some major upgrades in the past 6 months.

1) The geometry has been updated to reflect the latest (Illinois) calorimeter design.

2) The electron traceback code, used to help measure the muon phase space distribution, is now performed with GEANE. The traceback can be done through a non-uniform magnetic field and errors resulting from energy loss and scattering are now taken into account.

3) Muon loss monitor. A slight modification of the input directives allows the user to inject muons (instead of electrons) into the $\mathrm{g}-2$ ring. The lost muons are themselves produced by the tracking group.

4) The option of a non-zero value for the muon electric dipole moment has been introduced into G2GEANT. Seeing the effects of a plausible EDM requires more events than we can generate in a short period of time, but some of the systematics can be studied with fewer events and a grossly inflated EDM. 
5) Parameterized showers: Another GEANT-related package is GFLASH, which was developed by the H1 collaboration for the simulation of calorimeter showers. For some g-2 analyses it is crucial to have a fully simulated electromagnetic shower. For others a full simulation would be time-consuming and unnecessary, but scmething more sophisticated than simple energy smearing is required. GFLASH has parameterized shower shapes for its distributions, based ultimately on the work of Bock et al., but includes fluctuations. GFLASH, which was installed into G2GEANT this summer, speeds up event simulation by a factor of 100 or more.

6) Spill files: Data records for all timing devices (time over threshold and, if applicable, pulse width) can be written to an output file in a simple format, where the events from a single spill are kept together. The same has been done for the WFD pulse height measurements. The format of these output files will be standardized so that all data (Monte Carlo, lab bench, and g-2 experiment) looks essentially the same.

7) The original CERNIII fast rotation analysis code has been extracted from photocopies of the original, translated into Fortran 77, and interfaced with G2GEANT. It is now up and running at BU, and being used for muon phase-space measurement studies.

\section{Project Future Plans}

\section{CMD-2 at Novosibirsk}

The precise determination of the hadronic vacuum polarization contribution to the muon anomalous magnetic moment is the primary physics goal of the fall 1993 CMD-2 run. At Boston University, we will contribute manpower and material to this run, and will actively participate in the analysis of the data. In particular, the thesis work of BU graduate student Doug Brown will address the hadronic vacuum polarization determination. In addition, graduate student Edward Anashkin, who has several years of experience working with the CMD-2 apparatus and is both a hardware and software expert, will be resident at Boston University during the 1994 academic year. For more than a year we have been receiving recent data and up-to-date analysis programs from BINP, both via courier and through the Internet. Several messages each week also keep us apprised at Boston University of day-to-day operations at BINP. We also anticipate brief visits from individual CMD-2 collaborators in coordination with their visits to scientific conferences during 1994, as was the case in 1993. 
The large team of hardware and software professionals within the CMD-2 group constitutes an extremely valuable rescurce for E821, and we do well to promote projects in our common interest. To this end, we are proposing to provide VME-based data acquisition computing and electronics facilities to CMD-2, in coordination with our Yale and Brookhaven collaborators. The offline analysis computer capabilities which wo together provided in 1993 are forming an indispensable part of the CMD-2 hadronic vacuum polarization measurement. By providing our CMD-2 collaborators with the same Unix platform, operating system, and VME environment within which we will be developing DAQ software for E821, we can tap an extremely valuable source of skilled and experienced software and electronics experts. We have already demonstrated our capability to transfer software through the Internet, with the key element being the use of identical systems on both ends. E821 can derive maximal benefit from our CMD-2 collaborators by facilitating their ability to perform work which benefits E821 without having to leave BINP. For those Russians who will be able to visit BNL for short or extended visits, previous experience with E821 hardware and software will greatly enhance their ability to quickly contribute to our efforts.

This proposed method of US support was discussed by Asst. Prof. Worstell during his December 1992 visit to BINP, and in subsequent E-mail communication with CMD-2 collaborators. It has the approval and endorsement of Academician Lev Barkov and Dr. Boris Khazin, the CMD-2 team leaders. In particular, it answers Academician Barkov's request that we seek ways of working with our Russian collaborators which contribute to their capability to do science in Russia. The Internet link is a very powerful tool for facilitating collaborative effort, but off-site contributions to the very substantial E821 software effort will require BINP access to E821 hardware and software in its native environment. There is additional motivation for our CMD-2 collaborators to develop proficiency in online VME-based data acquisition software, which adds to our expectation that they will take full advantage of opportunities presented by this capability. With its latest highluminosity upgrade, CMD-2 is nearly deadtime-limited by its single-hit KLUKVA (BINP custom) DAQ electronics. The CMD-2 group recognizes the need for an electronics upgrade and for compatibility with systems in use abroad - in particular, with VME-based systems. Their current economic constraints have however restricted this effort to the planning stages. As Academician Barkov has pointed out, future plans are essential for a 
research group to maintain a coherent effort and to carry out current tasks well. The $\mathrm{g}-2$ WFD might be a prototype for multi-hit calorimeter readout, and the g-2 MTDC might be a prototype for multi-hit drift chamber readout; a CAMAC-based prototype WFD from Boston University is currently installed at CMD-2 as part of the luminosity monitoring system. Future plans within the CMD-2 group for CMD-3 development (high-luminosity with round beams) and ultimately a BINP $\phi$-meson factory will be subject to Russian economics, but BINP VME-based DAQ electronics and software development (which would form the basis of the CMD-3 detector upgrade) are very much in the interests of E821.

\section{Muon g-2 Front-End Electronics}

In 1993 we constructed prototypes of the front-end electronics systems for E821, and in 1994 we will comprehensively characterize the performance of these systems singly and in concert. If, as we have anticipated in our schedule, some systems do not initially meet E821 specifications for rate-independence, stability, and other systematic errors, we will iterate their design until they do. In addition, the VME interface for the MTDC system and the LORAN interface for the clock distribution system remain to be developed.

We have demonstrated that a Hamamatsu R1828 2" PMT can meet our specifications for high-rate gated stability. While this is a fine PMT, it may not be the least expensive PMT which can meet our specifications. Therefore, we plan to test a number of other candidate PMTs, which unfortunately requires the construction of a different high-rate gated base for each different PMT pin-out. In addition, our baseline design for the PMTs associated with the timing scintillators calls for less expensive 1" PMTs; we will therefore need to characterize candidate 1" PMTs to see whether they meet our specs as well, in order to minimize our overall PMT expenditures.

The WFD subsystem is probably nearest its final configuration. It has the least strict specifications on its required performance, since the muon $\mathrm{g}-2$ measurement is less sensitive to systematic errors in decay electron energy measurements than to timing errors. We must still demonstrate stable performance at high rates with our chosen PMT, and must optimize pulse shaping between the PMTs and WFDs (including the effects of dispersion in cable runs). Finally, the integration of the WFD data stream into the online DAQ system will provide the first exercise of the latter with E821-specific electronics.

The MTDC system has thus far only been tested at the component level, and has only been tested for basic functionality. Our CAMAC-interfaced module incorporates the 
essentials of the MTDC design, and it will be thoroughly tested. Next we wil' design. construct, and thoroughly test a VME-based module; these tests will be carried out singly and in combination with BNL de-randomizer circuits, and will ultimately include inputs from our LORAN-base clock system and from our selected PMTs operating at high rates. A full-system demonstration of stability and minimal systematic errors will form an arduous predecessor to the experiment itself. It will also establish our initial implementation of the calibration system for the experiment. The MTDC/de-randomizer system, collectively operating as did the "digitrons" in early muon g-2 measurements, form the heart of the electronics for the measurement. Consequently, our design for these systems retains the most flexibility but will require the most testing and careful development.

Finally, we must develop the LORAN-based high-stability clock system, and integrate it with a similar system being developed for the NMR precision magnetic field measurement system. Although locking onto LORAN will provide excellent long-term system stability, we will need to carefully characterize the system stability over millisecond time scales. The system must also be carefully isolated from effects due to the BNL AGS ramping cycle and injection into the storage ring. Small variations in clock rates which are not correlated with the storage ring cycle constitute a potential calibration nuisance, while effects correlated with the storage cycle constitute potential systematic errors for the muon g-2 measurement. While we do not anticipate any such variations above our 1 part in $10^{7}$ spec, we will nonetheless carefully demonstrate that no such variations exist. 


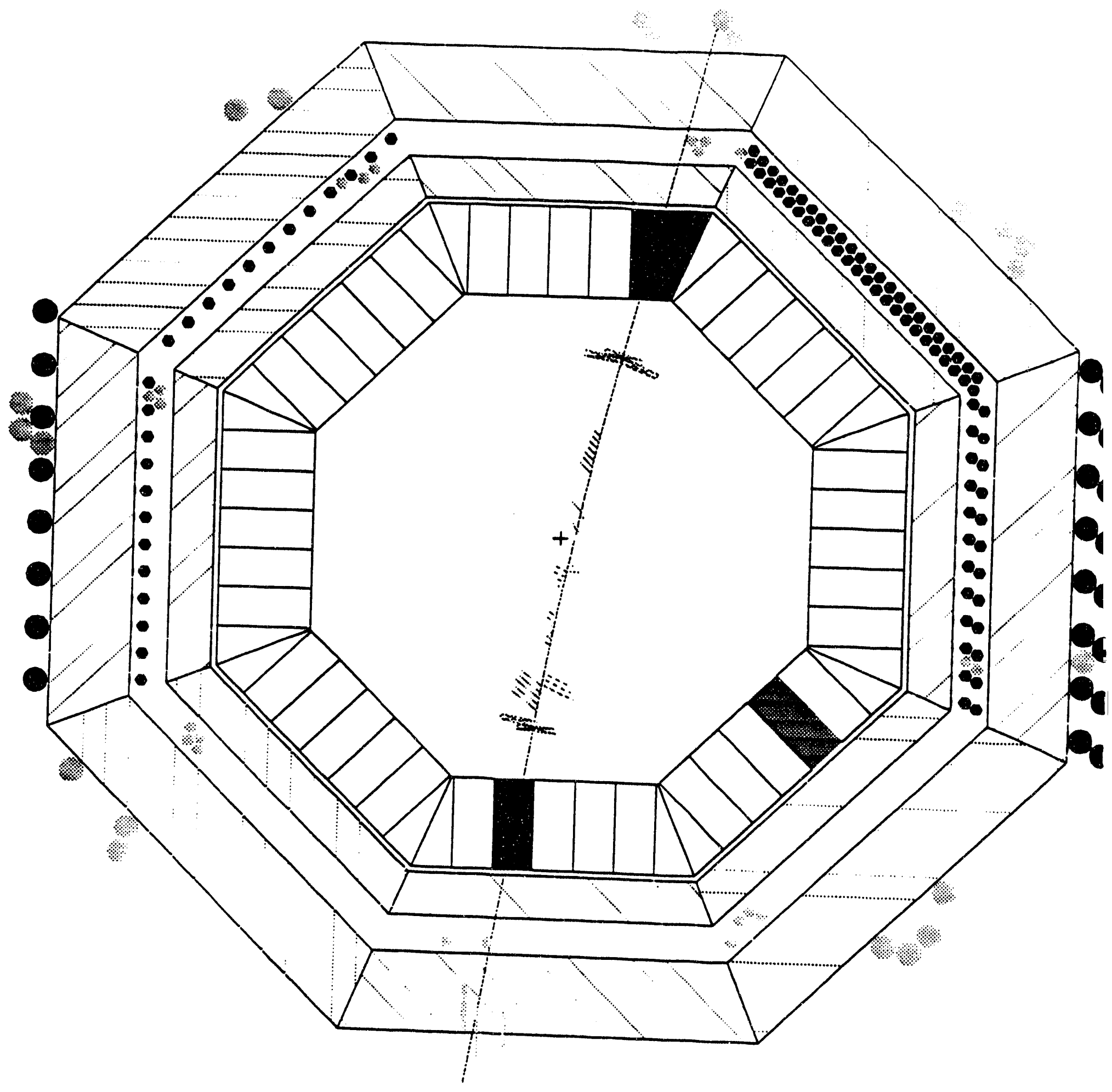

Figure 1. Display of an event within CMD-2, indicating hits on drift chamber cells, calorimeter crystals, $z$-strip wires, and muon tubes. 


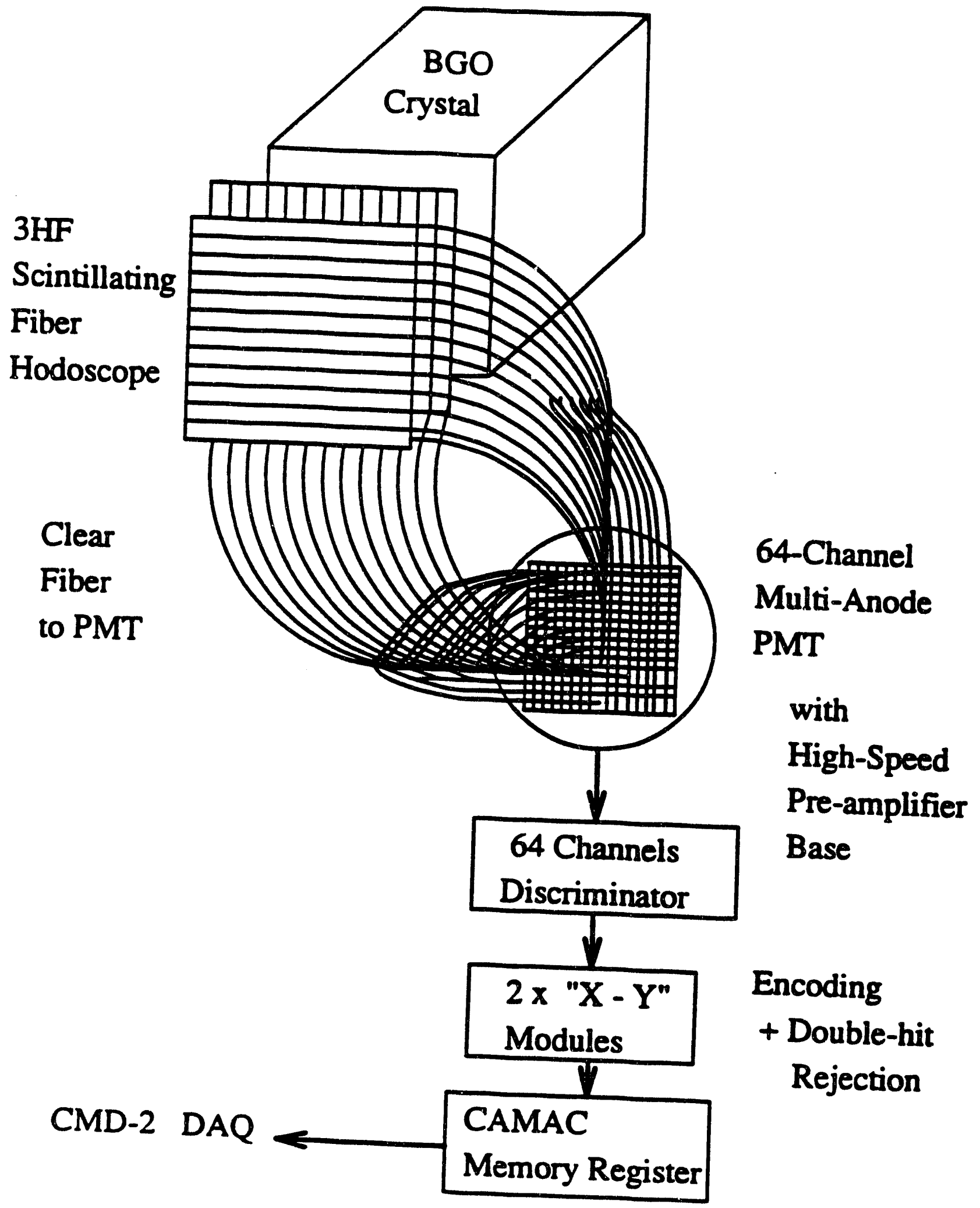

Figure 2. Schematic illustration of 64-channel Luminosity Monitor Position-Sensitive Detector (LM-PSD) constructed at Boston and installed at Novosibirsk. 

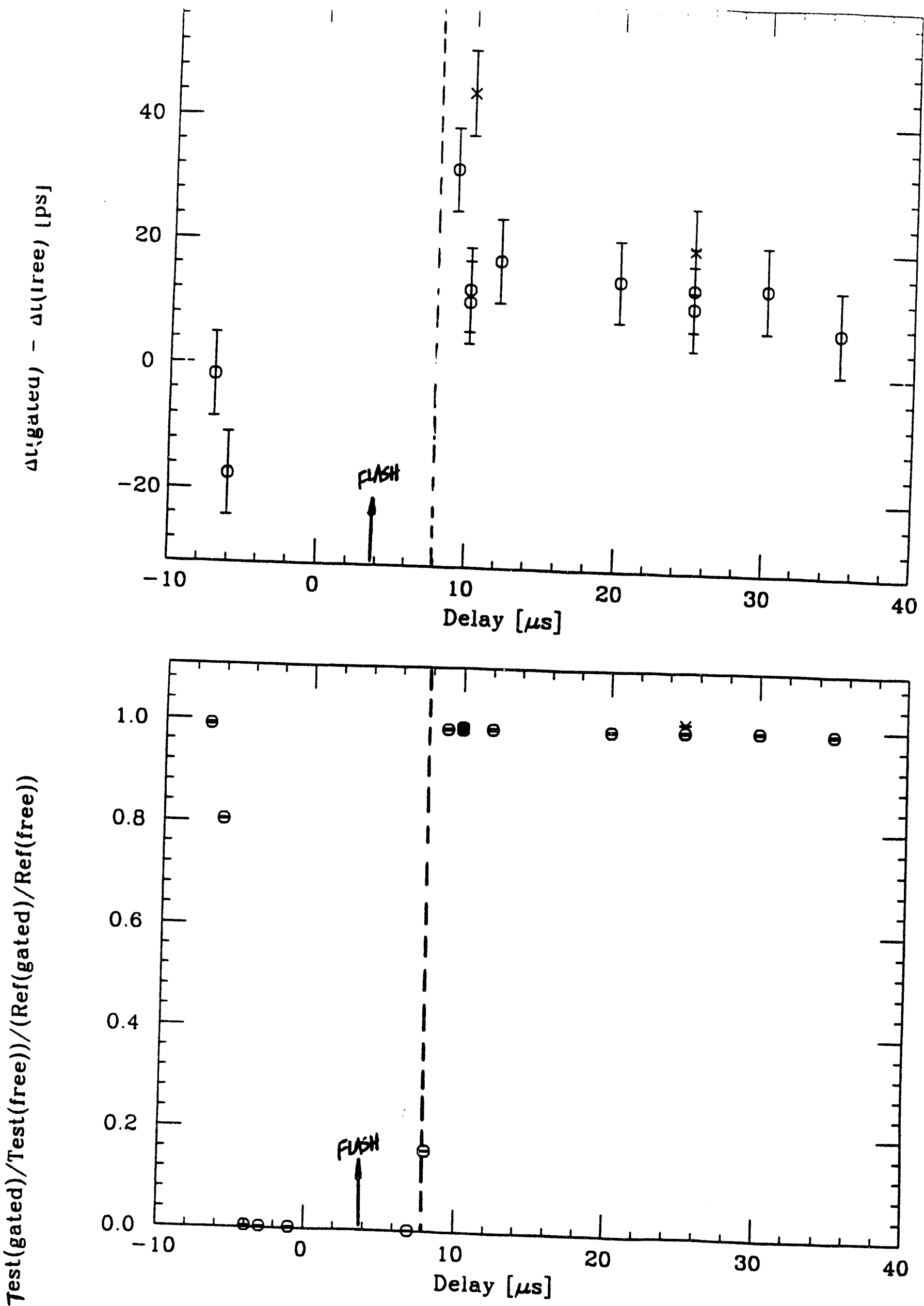

Figure 3. Bench test data illustrating rapid recovery of R1828 PMT with high-rate gated base developed at $\mathrm{BU}$. 

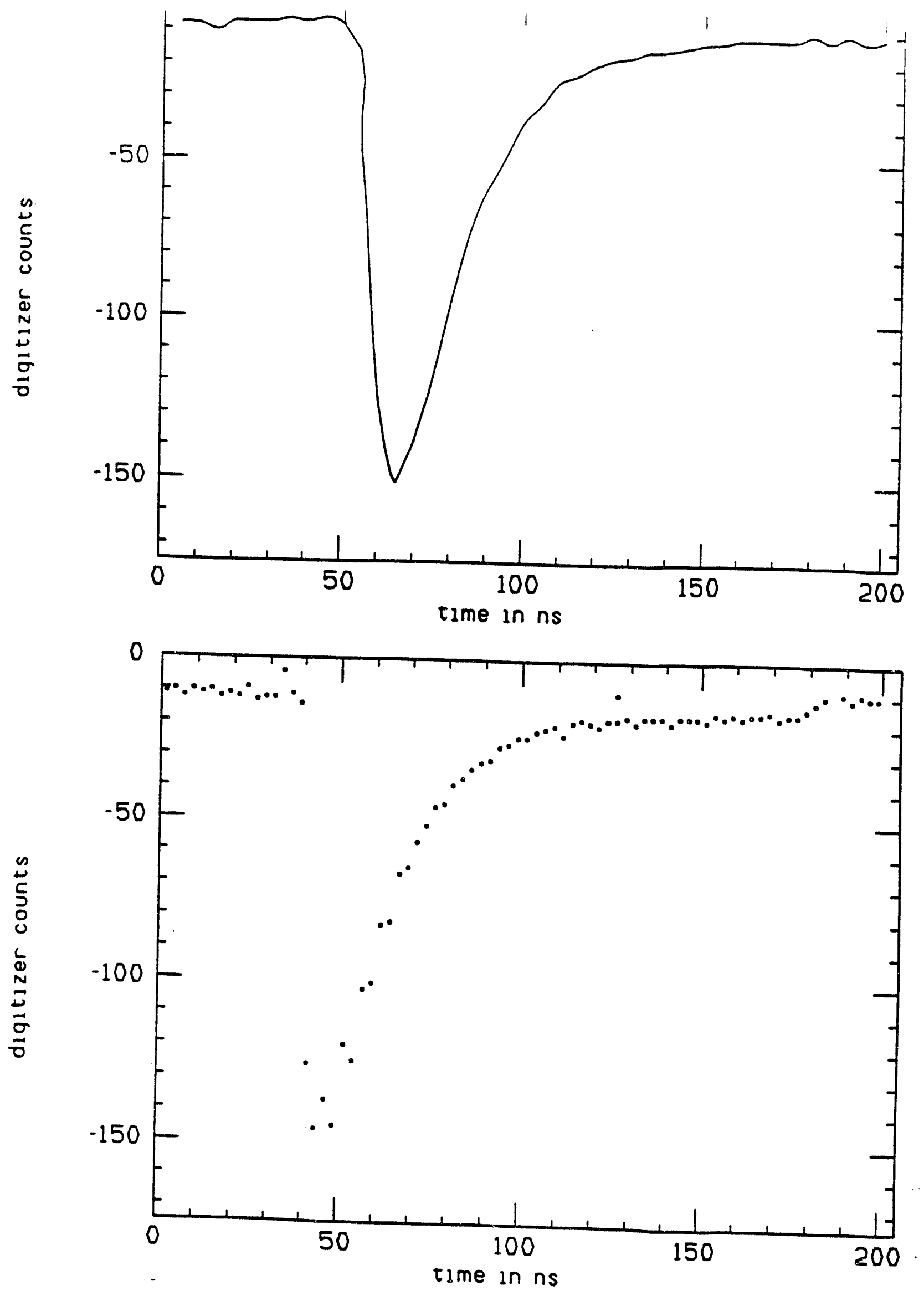

Figure 4. WFD data of cable-degraded PMT pulse from 1993 BNL beam test, showing interleaved readout (400 MSPS) of WFD developed at BU. 
Page 5-16 


\title{
TASK J: TOPICS IN PARTICLE PHYSICS
}

\author{
Faculty: $\quad$ Assistant Professor Andrew G. Cohen (P.I.) \\ Graduate Student: $\quad$ S. Myint
}

\section{Research Summary}

This report describes the research acconplished by Cohen since submission of his last OJI renewal in August, 1992. The report that follows is also included in Task E of this proposal.

With G. Ecker and A. Pich, Cohen has completed studies of the question of nonanalytic corrections to chiral perturbation theory in radiative kaon decays[1] for which there had been a discrepancy between theory and experiment[2][3]. Given a low energy effective Lagrangian describing the interactions of Goldstone (or pseudo-Goldstone) bosons, chiral perturbation theory is a simple technique for constructing Green functions as a power series expansion in powers of momenta (or symmetry breaking masses) over a scale which is typically $4 \pi f_{\pi}$. There are well-known corrections to this analytic expansion, known as chiral logarithms. These non-analytic terms are determined by unitarity, and are most easily computed by extracting the singular parts of Feynman diagrams constructed from the analytic expansion described above. In a chiral-perturbative expansion, these logarithms are small corrections to the leading terms. Cohen noted that there are circumstances where the non-analytic terms simulate the behavior of poles rather than logarithms, and these pole terms can be larger than the leading analytic parts. The contribution of this pole term may be computed for processes where it is dominant, and yields predictions for certain amplitudes in terms of other measured quantities. An example is $K_{L} \rightarrow \pi^{0} \gamma \gamma$, which may be related to the measured $K_{L} \rightarrow \pi^{0} \pi^{+} \pi^{-}$amplitude. Ecker, Pich and Cohen have used this idea to improve the theoretical relation between the experimental $K_{L} \rightarrow 3 \pi$ amplitude and 
the rate and spectrum for $K_{L} \rightarrow \pi^{0} \gamma \gamma$. The result is in quite good agreement, and resolves the discrepancy between the experimental data and previous theoretical calculations.

The question of the origin of the baryon excess of the observable universe, is one of the most profound we face. Particle physicists have come to understand some of the ingredients necessary for generating the baryon excess: fundamental baryon number non-conservation (either explicitly or spontaneously broken), CP violation, and out-of-thermal-equilibrium processes[4]. In the late 1970s it was shown how these three ingredients could arise in the context of Grand Unified Theories (GUTs) and lead to a baryon excess in the present epoch[5]. Indeed the presence of baryon violation and the fact that it leads to a baryon excess today remains one of the most attractive features of GUTs. However, it is difficult to obtain a baryon excess of the size observed today without excessively complicating or fine-tuning the GUT. In addition, the absence of proton decay at the rate predicted by the simplest GUTs has cast some doubt on this explanation of the origin of the baryon excess.

Cohen, Kaplan and Nelson have used spontaneous baryogenesis to construct models that can produce a baryon asymmetry at the weak phase transition. In Ref. [6] they developed a new method for generating a baryon asymmetry that, rather than avoiding fast weak interaction baryon violation at high temperature, makes direct use of it. The key problems for using weak baryon violation are to maintain departure from thermal equilibrium at the weak scale and to find a source of CP violation to drive baryon violation in the right direction. In one model, the departure from equilibrium was accomplished by having the weak phase transition be weakly first order. The phase transition then occurs through bubble nucleation, with a non-equilibrium separation of phases. Cohen, Kaplan and Nelson applied this idea to a standard model with extra Higgs doublets[7].

Cohen and Nelson have investigated baryogenesis in supersymmetric models[8]. In order for the minimal supersymmetric standard model to produce the observed asymmetry, all of the parameters (such as the gaugino masses, higgsino masses, phases, etc.) must lie within very narrow ranges. This yields a rather definite prediction for the electric dipole moment of the neutron and, with some assumptions about squark and slepton masses, for the electric dipole moment of the electron. These constraints can be relaxed in nonminimal models through the addition of gauge singlets which can change the details of the phase transition.

Cohen, Kaplan and Nelson have completed a long review of the subject of electroweak baryogenesis for Annual Reviews in Nuclear and Particle Science[9]. This review covers the basic concepts of baryon violation in the standard model, details of phase transitions 
in the early universe with emphasis on the electroweak transition, and a lengthy discussion of the generation of the baryon asymmetry at such a transition.

Along with R. S. Chivukula, M. Luke and M. Savage, Cohen has reconsidered the detection of color-neutral techni-bound states composed of colored constituents as dark matter[10]. Previously Nussinov suggested that such objects might be visible in dark matter detectors[11]. He used a non-relativistic quark model to compute the interaction of these objects in such detectors. However it now appears that this calculation was in error; these authors realized that the interaction of such objects would be governed by matrix elements of the gluon field-strength squared, and that such matrix elements can be calculated using ward-identities of QCD along with heavy particle effective field theory. The result is that the cross section for a techniparticle of this sort on ordinary matter is 7 orders of magnitude smaller than Nussinov's estimate, and is not observable in current (or proposed) dark matter detectors.

Cohen is in the process of completing a lengthy project with S. Coleman concerning the proper definition of time dependent quantities in quantum field theory at finite temperature. In particular they have considered the question of the decay of a metastable extended configuration in a weakly coupled field theory. The notion of the decay time of such a configuration is actually quite subtle-a priori it is not even clear that a universal "lifetime" exists. That is, unlike the zero temperature case (or the case of quasiparticles at finite temperature), it is not obvious that there exists an exponential decay law that is measured in an appropriate gedanken experiment, where the exponential rate is independent of the way in which the initial state is prepared. Indeed, most attempts at calculations of these quantities have assumed a very particular initial state; the final result for the lifetime may depend sensitively on this initial form. This would not be a disaster, but it would mean that in any calculation one would have to decide precisely what the initial configuration should be (for example, in the early universe the configuration at a phase transition may depend on the past history of the universe). However Coleman and Cohen have shown that for a broad class of initial configurations (which include the physically relevant cases) there is indeed a universal exponential decay law corresponding to an observable in an appropriate experimental arrangement. This observable is not what is usually calculated, and indeed the relationship between this observable and that usually calculated is obscure. In addition they have developed a formalism appropriate for semi-classical approximations to the thermal decay time. This work is currently in preparation. 
During the coming year Cohen plans to continue his work on baryogenesis. Recent suggestions by Shaposhnikov and Ferrer that the observed baryon asymmetry may arise in the standard model with no new source of CP violation has generated a great deal of controversy. Currently Cohen is investigating these claims and hopes to provide a reliable estimate of the maximum baryon asymmetry produced in this model.

Cohen and Coleman continue their investigations of finite time-temperature effects in weakly coupled field theories. They hope to eventually calculate such rates in complicated field theories such as the standard model.

Along with R. Rohm Cohen plans to complete work on a $1+1$ dimensional model of baryon violation. By considering the $C P^{N}$ sigma model with soft symmetry breaking they hope to provide a laboratory for weak instantons in a simpler context. Previous $1+1$ dimensional analogs do not adequately reflect the features of the true $3+1$ dimensional theory; hopefully this particular model will prove more reliable for predicting $3+1$ dimensional physics.

With A. DeRujula Cohen is considering the possibility that experiments at HERA might be able to distinguish the contribution of charm states to the structure function of the proton. Using a combination of perturbative QCD, Regge theory and wishful thinking they are attempting to estimate this effect. 


\section{References}

[1] A. Cohen, G. Ecker and A. Pich, "Unitarity and $K_{L} \rightarrow \pi \gamma \gamma$," BUHEP-93-5 to appear in Physics Letters.

[2] NA31 Collab., G.D. Barr et al., Phys. Lett. B284, 440 (1992).

[3] E731 Collab., V. Papadimitriou et al., Phys. Rev.D44, 573 (1991).

[4] A. Sakharov, Zh. Eksp. Teor. Piz. Pis'ma 5, 32 (1967).

[5] S. Dimopoulos and L. Susskind, Phys. Rev. D18, 4500 (1978); M. Yoshimura, Phys. Rev. Lett. 41, 281 (1978); (E) 42, 746 (1978); D. Toussaint, S. Treiman, F. Wilczek and A. Zee, Phys. Rev. D19, 1036 (1979).

[6] A. Cohen, D. Kaplan and A. Nelson, Phys. Lett. B245, 561 (1990).

[7] A. Cohen, D. Kaplan and A. Nelson, Nucl. Phys. B373, 453 (1992).

[8] A. Cohen and A. Nelson, Phys. Lett. B297, 111 (1992).

[9] Progress in Electroweak Baryogenesis, BUHEP-93-4, to appear in Annual Review in Nuclear and Particle Science (with D. Kaplan and A. Nelson).

[10] R.S. Chivukula, A. Cohen, M. Luke and M. Savage Phys. Lett. B298, 380 (1993).

[11] S. Nussinov, Phys. Lett. B279, 111 (1992). 


\section{TASK K: HADRON CALORIMETRY FOR THE GEM DETECTOR AT THE SSC}

Faculty:

Research Faculty:

Research Associate:

Associated Faculty:

Collaborating Institutions:
Professor L. R. Sulak (P.I.)

Associate Professor James L. Stone

Associate Professor James Sullivan

S. Dye

R. Carey

Associate Professor J. P. Miller (NSF)

Professor B. L. Roberts (NSF)

Drexel, Fairfield, Iowa, Louisiana State, Michigan State, Mississippi, Mississippi State, Tennessee, Texas A\&M, Texas Tech, UC-San Diego

National Laboratory Affliates: C. S. Draper Lab, Oak Ridge, Lawrence Livermore

Project Summary

The GEM Collaboration at the SSC has chosen a hybrid hadron calorimeter comprising, in the barrel, a $6.5 \lambda$ noble liquid, $\mathrm{Kr}$, calorimeter and a $4.5 \lambda$ scintillating fiber external calorimeter. This is discussed in the GEM Technical Design Report (SSCL-SR1219) submitted on April 30, 1993. The current plan for GEM lists three tasks as highest priority:

External barrel calorimeter and central detector support. The installation in the experiment hall must start in October 1996.

Beam test and calibration at FNAL in March 1995. Delivery and installation of the first pre-production modules must commence in November 1994.

Superconducting magnet. 
We are directly responsible for major components of the first two tasks on this list. The schedule implies that the two modules needed for the beam test should be pre-production prototypes and must be built here in the US. Our institution is unique in its prior experience in building copper and scintillator modules due to prior TNRLC grants. The overall SCIGEM (external scintillating calorimeter for GEM) plan and our role at Boston University are driven by the need to meet the schedule milestones listed above. Particular tasks at Boston planned for the coming year:

Manufacturing engineering: To be ready for immediate construction after the anticipated approvals in January 1994, our Scientific Instrumentation Facility will supply detailed manufacturing engineering support for the overall design in collaboration with ORNL and develop necessary fixturing. Actual fabrication occurs here in the following year. Also, alternative techniques for the machining of optical tiles will be evaluated in conjunction with the University of Mississippi and ORNL.

Electronics: Our Electronic Design Facility are producing and testing prototype highvoltage supplies in conjunction with collaborators at the University of Quito, Ecuador. They are also developing front end electronics (with ORNL) to mate with the noble liquid measurement chains. Although, these developments will continue for more than one year, a few channels are essential for inclusion in the FNAL tests by January 1995.

Magnetic field sensitivity tests: We are continuing a program of photosensor evaluation in strong magnetic fields. The detailed performance is measured in various orientations and under various operating conditions. These tests are conducted in an 11.5 inch high-field solenoid magnet at the Francis Bitter Magnet Laboratory of MIT. These studies are done in close collaboration with the University of Iowa.

Calibration and monitoring: Conceptual design of the calibration system, integration into engineering design, and bench checkout of a piezoelectric radioactive source system are being carried out in conjunction with Iowa.

Simulation studies: Work on optimization of muon trigger and Monte-Carlo study of light collection in tile assemblies is proceeding with ORNL and Mississippi.

SCIGEM: Overall coordination (with ORNL) of the SCIGEM effort and working with potential international partners is a major responsibility of Boston University. Dr. Sulak is Chairman of the International Committee of GEM. 
The SSCintCal group communicates through a bi-weekly telephone conference initiated from Boston University. We conduct regular meetings every 6 weeks at the SSC Laboratory, in conjunction with both GEM collaboration council and GEM calorimeter subgroup meetings. Additional meetings are held at ORNL with our engineering colleagues and at FNAL to coordinate plans for the beam tests there. The operations budget requested under Task $\mathrm{K}$ covers the teleconferencing and meeting costs associated with this coordination activity. 


\section{TASK N: TOPICS IN ELEMENTARY PARTICLE PHYSICS \\ Faculty: \\ Assistant Professor R. Sekhar Chivukula (co-PI) \\ Graduate Student: $\quad$ B. Balaji}

\section{Research Accomplishments}

This report describes the research accomplished by Chivukula since the submission of the last OJI renewal in August, 1992. The descriptions that follow are also included in Task E of this proposal.

\section{A. Electroweak Symmetry Breaking}

Expanding on previous work [1], Chivukula (with M. Golden (Harvard), D. Kominis, and M. V. Ramana) calculated [2] the production rate of gauge-boson pairs at the SSC in a model with a "hidden" electroweak symmetry breaking sector. They showed that the signal of electroweak symmetry breaking is lower than the background and that one cannot necessarily rely on gauge boson pairs as a signal of the dynamics of symmetry breaking. These results were also reported [3] at the conference on "High Energy Physics with Colliding Beams" held at Yale University, Oct. 2-3, 1992.

With Golden and E. H. Simmons (Harvard), Chivukula considered [4] the constraints that critical dynamics places on models with a top quark condensate or strong extended technicolor (ETC). These models require that chiral-symmetry-breaking dynamics at a high energy scale plays a significant role in electroweak symmetry breaking. In order for there to be a large hierarchy between the scale of the high energy dynamics and the weak scale, the high energy theory must have a second order chiral phase transition. If the transition is second order, then close to the transition the theory may be described in terms of a low-energy effective Lagrangian with composite "Higgs" scalars. However, scalar theories in which there are more than one $\Phi^{4}$ coupling can have a first order phase transition instead, due to the Coleman-Weinberg instability. They showed, therefore, that top-condensate or strong ETC theories in which the composite scalars have more than one $\Phi^{4}$ coupling cannot always support a large hierarchy. In particular they concluded that, 
if the Nambu-Jona-Lasinio model solved in the large- $N_{c}$ limit is a good approximation to the high-energy dynamics, then these models will not produce acceptable electroweak symmetry breaking.

With Kominis, Chivukula examined [5] perturbatively two-Higgs-doublet extensions of the standard model in the context of the suspected triviality of theories with fundamental scalars. Requiring the model to define a consistent effective theory for scales below a cutoff of $2 \pi$ times the largest mass of the problem, as motivated by lattice investigations of the one-Higgs-doublet model, they obtained combined bounds for the parameters of the model. They found upper limits of $470 \mathrm{GeV}$ for the mass of the light $C P$-even neutral scalar and 650-700 GeV for the other scalar masses.

With his student, V. Koulovassilopoulos, Chivukula investigated [6] the properties of a non-standard Higgs boson. Because of the triviality of the usual scalar symmetry breaking sector, if the Higgs mass is approximately $600 \mathrm{GeV}$ or higher, there must be additional dynamics at a scale $\Lambda$ which is less than a few TeV. In this case the properties of the Higgs resonance can differ substantially from those predicted by the standard model. They constructed a chiral Lagrangian description of a theory with a non-standard Higgs boson and analyzed the features of a theory with such a resonance coupled to the Goldstone bosons of the breaking of $S U(2) \times U(1)$. The effective Lagrangian they constructed describes the most general theory in which the Higgs and the Goldstone bosons are the only particles with a mass small compared to $\Lambda$. They computed the leading chiral corrections to the decay width of the Higgs boson and the contribution to the Peskin-Takeuchi parameter $S$ and presented results for the corrections to Goldstone boson scattering. A longer paper with a more detailed description of the calculations and an analysis of a specific model is in preparation.

\section{B. Nonoblique Corrections to $Z \rightarrow b \bar{b}$}

Expanding on previous work with Simmons and S. B. Selipsky [7], Chivukula (with Simmons, J. Terning (Yale), and E. Gates (Chicago)) investigated [8] how a slowly running technicolor coupling affects non-oblique corrections to the $Z b \bar{b}$ vertex from extended technicolor dynamics. They have shown that, for a top quark $100 \mathrm{GeV}$ or heavier, there would be effects detectable with experiments at LEP. 


\section{QCD with Many Light Flavors}

With Golden and M. Dugan, Chivukula [9] investigated the limits of chiral perturbation theory in a QCD-like theory with many light flavors. The chiral Lagrangian for Goldstone boson scattering is a power series expansion in numbers of derivatives. Each successive term is suppressed by powers of a scale, $\Lambda_{\chi}$, which must be less than of or$\operatorname{der} 4 \pi f / \sqrt{N}$ where $f$ is the Goldstone boson decay constant and $N$ is the number of flavors. The chiral expansion therefore breaks down at or below $4 \pi f / \sqrt{N}$. They argued that the breakdown of the chiral expansion is associated with the appearance of physical states other than Goldstone bosons and speculated that, since the bound on $\Lambda_{\chi}$ falls as $N$ increases, the masses of resonances will decrease relative to $f_{\pi}$ at least as fast as $1 / \sqrt{N}$.

Expanding on these points, Chivukula (with Golden and Dugan) reevaluated [10] the calculations of radiative corrections in technicolor theories. Radiative corrections to electroweak parameters in technicolor theories may be evaluated by one of two techniques: either one estimates spectral function integrals using scaled QCD data, or one uses naive dimensional analysis with a chiral Lagrangian. The former yields corrections to electroweak parameters proportional to the number of flavors and the number of colors, while the latter is proportional to the number of flavors squared and is independent of the number of colors. They resolved this apparent contradiction by showing that the spectrum of technicolor one obtains by scaling QCD data to high energies is unlikely to resemble that of an actual technicolor theory. The resonances are likely to be much lighter than naively supposed and the radiative corrections to electroweak parameters may be much larger. They also argued that much less is known about the spectrum and the radiative corrections in technicolor than was previously believed.

\section{Cosmology}

With M. Luke and M. Savage (both of UCSD), and A. Cohen, Chivukula estimated [11] the cross section for the scattering of a slow, color-neutral technibaryon made of colored constituents with nuclei. Contrary to previous estimates, they found a cross section of order $A^{2} 10^{-45} \mathrm{~cm}^{2}$, where $A$ is the atomic number of the nucleus. They concluded that, even if technibaryons constitute the dark matter in the galactic halo, this is too small to be detected in future underground detectors. 


\section{References}

[1] "Hiding the Electroweak Symmetry Breaking Sector" (with M. Golden), Phys. Lett. B 267, 233 (1991).

[2] "Phenomenology of a Hidden Symmetry Breaking Sector" (with M. Golden, D. Kominis, and M. V. Ramana), Phys. Lett. B 293, 400 (1992).

[3] "Hiding the Electroweak Symmetry Breaking Sector" (with M. Golden, D. Kominis, and M. V. Ramana), Proceedings of High Energy Physics with Colliding Beams (Yale University, New Haven, Oct. 2-3, 1992); Boston University preprint BUHEP-92-34.

[4] "Critical Constraints on Chiral Hierarchies" (with M. Golden and E. H. Simmons), Physical Review Letters 70, 1587 (1993).

[5] "Triviality Bounds in Two-Doublet Models" (with D. Kominis), Phys. Lett. B 304, 152 (1993).

[6] "Phenomenology of a Non-Standard Higgs" (with V. Koulovassilopoulos), Boston University preprint BUHEP-93-7, and Phys. Lett. B in press.

[7] "Non-Oblique Effects in the $Z b \bar{b}$ Vertex from ETC Dynamics" (with S. B. Selipsky, and E. H. Simmons), Phys. Rev. Lett. 69, 575 (1992).

[8] "Walking Technicolor and the $Z b \bar{b}$ Vertex" (with E. Gates, E. H. Simmons, and J. Terning), Boston University preprint BUHEP-93-11, and Phys. Lett. B in press.

[9] "Analyticity, Crossing Symmetry and the Limits of Chiral Perturbation Theory" (with M. J. Dugan and M. Golden), Phys. Rev. D47, 2930 (1993).

[10] "Electroweak Corrections in Technicolor Reconsidered" (with M. Golden and M. J. Dugan), Phys. Lett. B 292, 435 (1992).

[11] "A Comment on the Strong Interactions of Color-Neutral Technibaryons" (with A. Cohen, M. Luke, and M. Savage), Phys. Lett. B 298, 380 (1993). 


\title{
TASK RS: RESEARCH SUPPORT
}

\author{
Project Director: Drofessor J. Scott Whitaker \\ Grant Administrator: Rachel Meisel
}

\section{Discussion}

The research program described in this proposal is a serious, large-scale effort and requires professional administration. The Research Support task represents the administrative support necessary for the accomplishment of the research goals of this program. The seven projects described earlier in this document encompass the research activities of twelve faculty members, nine research faculty and research associates, and fifteen graduate students. Our administrator, Rachel Meisel, was hired in August 1989 to work full-time on the administration of the projects described in this proposal. The administrator is involved in every aspect of the program. She is the principal liaison with the Boston University purchasing, personnel, and accounting departments.

Meisel is essential to the successful operation of this grant. She has been very effective in using the resources of Boston University in support of this research program. With Meisel shouldering the major burden of grant administration, the scientific productivity of the principal investigators has increased. The proposed budget consists of salary support and fringe benefits for the administrator and limited operating support for administrative functions. 

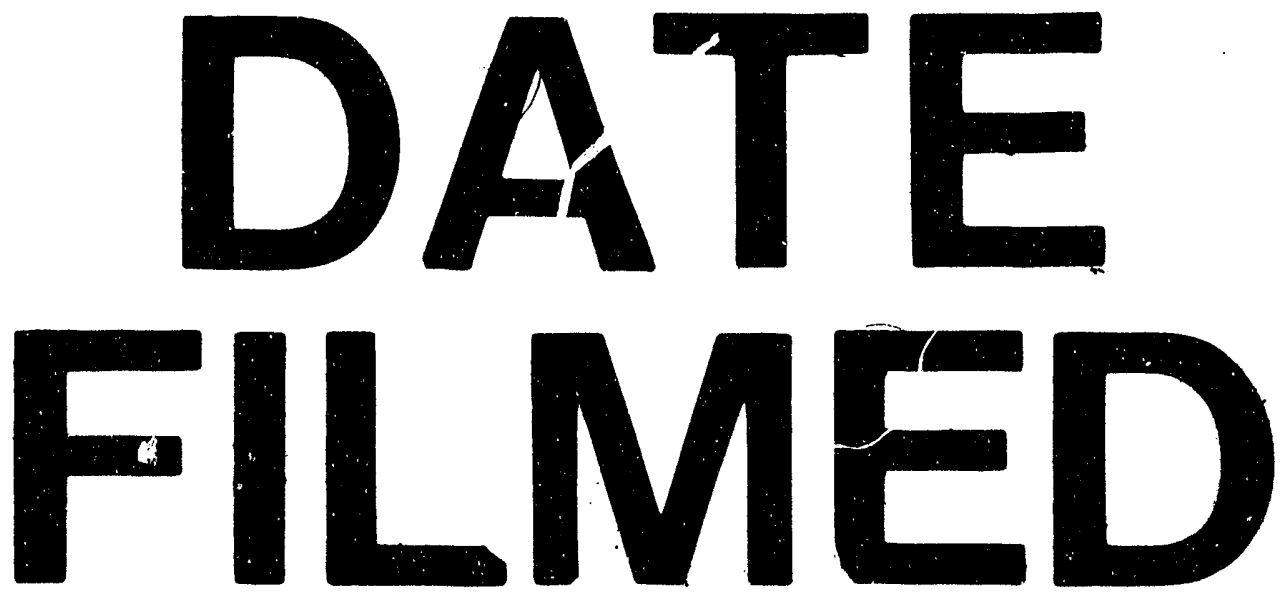

$10 / 12 / 93$ 
